Florida International University FIU Digital Commons

$11-9-2012$

\title{
Positron Emission Tomography (PET) Tumor Segmentation and Quantification: Development of New Algorithms
}

Ruchir N. Bhatt

Florida International University, rbhat002@fiu.edu

DOI: $10.25148 /$ etd.FI12121004

Follow this and additional works at: https://digitalcommons.fiu.edu/etd

\section{Recommended Citation}

Bhatt, Ruchir N., "Positron Emission Tomography (PET) Tumor Segmentation and Quantification: Development of New Algorithms" (2012). FIU Electronic Theses and Dissertations. 798.

https://digitalcommons.fiu.edu/etd/798 


\section{FLORIDA INTERNATIONAL UNIVERSITY}

Miami, Florida

POSITRON EMISSION TOMOGRAPHY (PET) TUMOR SEGMENTATION AND QUANTIFICATION: DEVELOPMENT OF NEW ALGORITHMS

A dissertation submitted in partial fulfillment of the requirements for the degree of DOCTOR OF PHILOSOPHY

in

BIOMEDICAL ENGINEERING

by

Ruchir Bhatt 
To: Dean Amir Mirmiran

College of Engineering and Computing

This dissertation, written by Ruchir Bhatt, and entitled Positron Emission Tomography (PET) Tumor Segmentation and Quantification: Development of New Algorithms, having been approved in respect to style and intellectual content, is referred to you for judgment.

We have read this dissertation and recommend that it be approved.

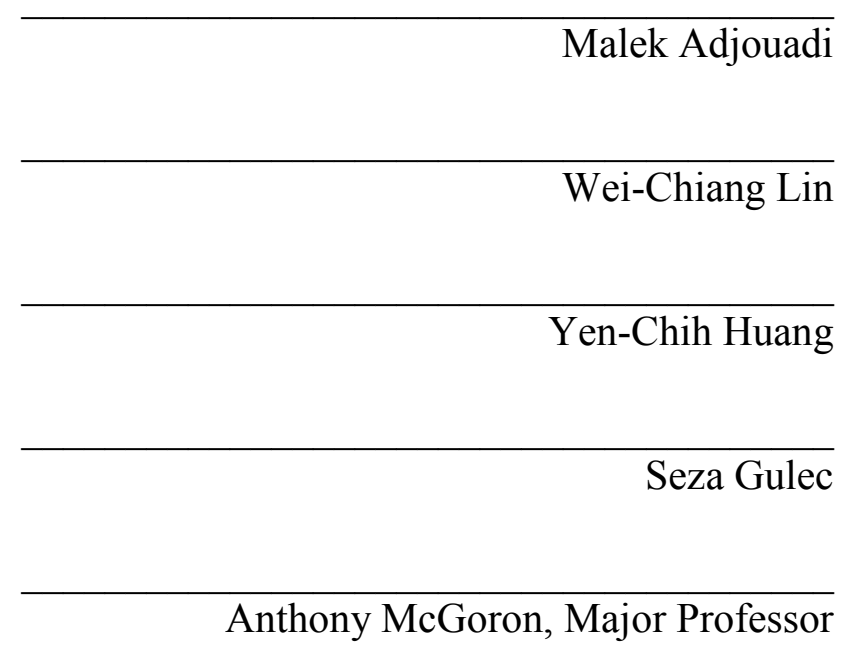

Date of Defense: November 9, 2012

The dissertation of Ruchir Bhatt is approved.

Dean Amir Mirmiran College of Engineering and Computing

Dean Lakshmi N. Reddi University Graduate School

Florida International University, 2012 
ABSTRACT OF THE DISSERTATION

POSITRON EMISSION TOMOGRAPHY (PET) TUMOR SEGMENTATION

AND QUANTIFICATION: DEVELOPMENT OF NEW ALGORITHMS

by

Ruchir Bhatt

Florida International University, 2012

Miami, Florida

Professor Anthony McGoron, Major Professor

Tumor functional volume (FV) and its mean activity concentration (mAC) are the quantities derived from positron emission tomography (PET). These quantities are used for estimating radiation dose for a therapy, evaluating the progression of a disease and also use it as a prognostic indicator for predicting outcome. PET images have low resolution, high noise and affected by partial volume effect (PVE). Manually segmenting each tumor is very cumbersome and very hard to reproduce. To solve the above problem I developed an algorithm, called iterative deconvolution thresholding segmentation (IDTS) algorithm; the algorithm segment the tumor, measures the FV, correct for the PVE and calculates mAC. The algorithm corrects for the PVE without the need to estimate camera's point spread function (PSF); also does not require optimizing for a specific camera. My algorithm was tested in physical phantom studies, where hollow spheres $(0.5-16 \mathrm{ml})$ were used to represent tumors with a homogeneous activity distribution. It was also tested on irregular shaped tumors with a heterogeneous activity profile which were acquired using physical and simulated phantom. The physical phantom studies were performed with different signal to background ratios (SBR) and 
with different acquisition times (1-5 min). The algorithm was applied on ten clinical data where the results were compared with manual segmentation and fixed percentage thresholding method called $\mathrm{T} 50$ and $\mathrm{T} 60$ in which $50 \%$ and $60 \%$ of the maximum intensity respectively is used as threshold. The average error in FV and $\mathrm{mAC}$ calculation was $30 \%$ and $-35 \%$ for $0.5 \mathrm{ml}$ tumor. The average error $\mathrm{FV}$ and $\mathrm{mAC}$ calculation were $\sim 5 \%$ for $16 \mathrm{ml}$ tumor. The overall $\mathrm{FV}$ error was $\sim 10 \%$ for heterogeneous tumors in physical and simulated phantom data. The FV and $\mathrm{mAC}$ error for clinical image compared to manual segmentation was around $-17 \%$ and $15 \%$ respectively. In summary my algorithm has potential to be applied on data acquired from different cameras as its not dependent on knowing the camera's PSF. The algorithm can also improve dose estimation and treatment planning. 


\section{TABLE OF CONTENTS}

CHAPTER

PAGE

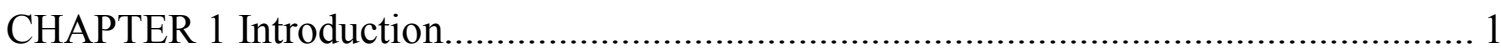

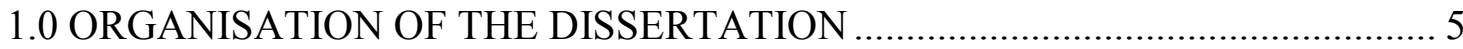

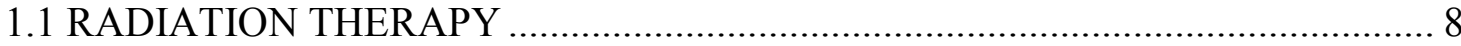

1.1.2 SELECTIVE INTERNAL RADIATION THERAPY ……………………..... 8

1.1.3 EXTERNAL RADIATION THERAPY ………….................................... 9

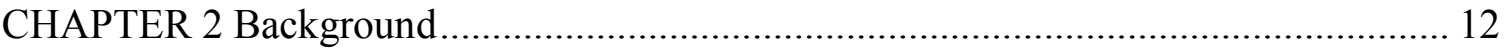

2.1 FUNCTIONAL VOLUME ESTIMATION ALGORITHM................................... 12

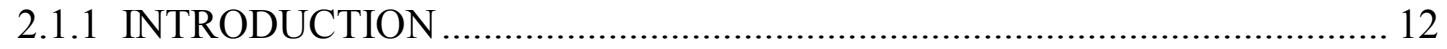

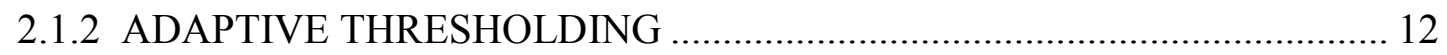

2.1.3 ACTIVE CONTOUR SEGMENTAITON ………................................... 12

2.1.4 GRADIENT BASED SEGMENTATION ……….................................... 13

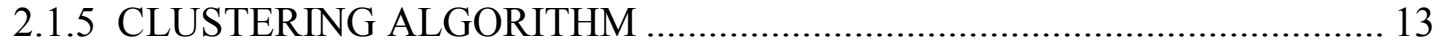

2.1.6 FUZZY BASED SEGMENTATION …………....................................... 14

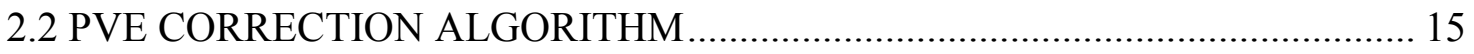

2.2.1 INTRODUCTION ……………………………………………….... 15

2.2.2 ANATOMICAL DATA BASED PARTIAL VOLUME EFFECT

CORRECTION...................................................................................... 15

2.2.3 DECONVOLUTION BASED PARTIAL VOLUME EFFECT CORRECTION 16

CHAPTER 3 Tumor Segmentation and Quantification Algorithm …….......................... 17

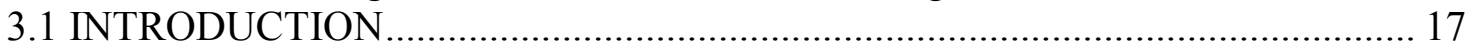

3.2 HISTOGRAM THRESHOLDING ALGORITHM ……..................................... 19

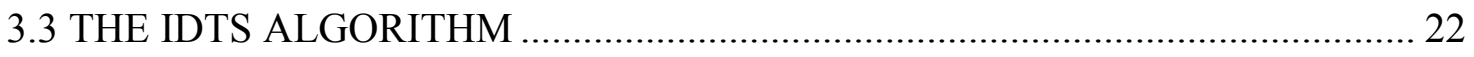

3.3.1 DECONVOLUTION METHOD ................................................................. 23

3.3.2 MODIFIED HISTOGRAM THRESHOLDING (MHT) ALGORITHM ........ 23

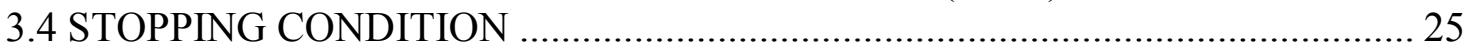

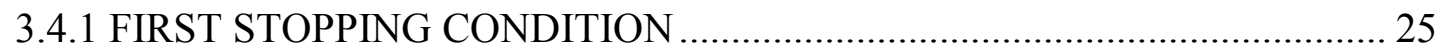

3.4.2 SECOND STOPPING CONDITION ........................................................ 26

3.5. MAXIMUM INTENSITY CORRECTION ……….......................................... 28

3.6. ALTERED HISTOGRAM THRESHOLDING (FOR CLINICAL IMAGES)...... 33

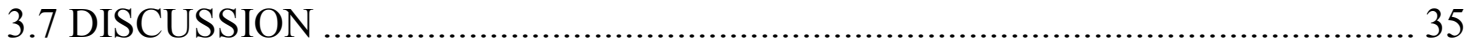

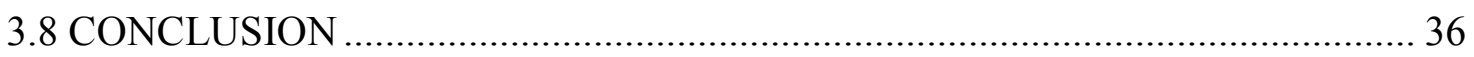

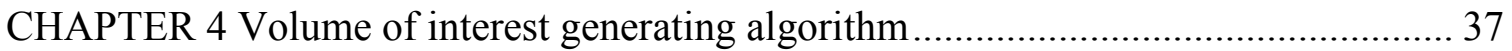

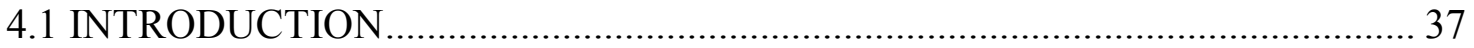

4.2 PROBLEM WITH DRAWING ONE REGION OF INTEREST ............................ 37

4.3 VOLUME OF INTEREST GENERATING ALGORITHM ................................ 41

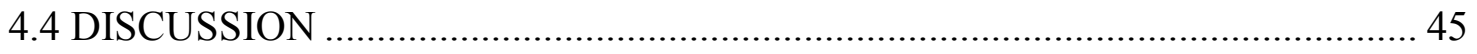

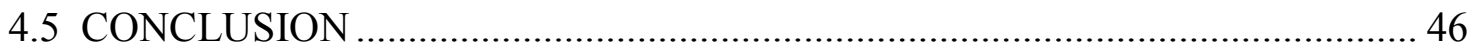




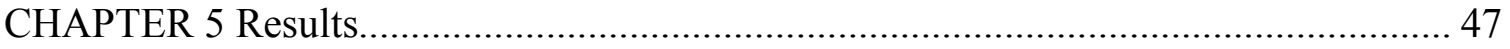

5.1 PHYSICAL PHANTOM STUDIES (HOMOGENEOUS TUMORS) ................... 47

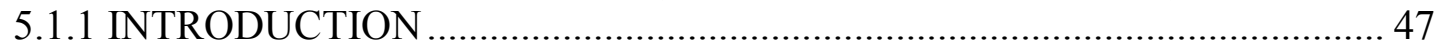

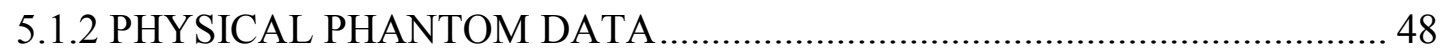

5.1.3 TESTING THE IMPACT OF MANUAL DRAWING OF REGION OF

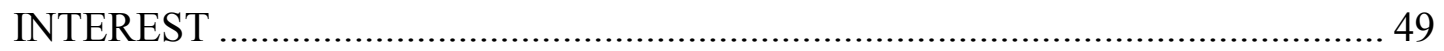

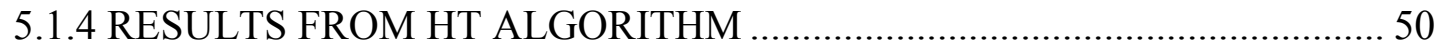

5.1.5 THE FINAL FV AND RC AND ROBUSTNESS OF THE IDTS

ALGORITHM .............................................................................................. 52

5.1.6 STATASTICAL ANALYSIS OF PHANTOM STUDIES ..............................59

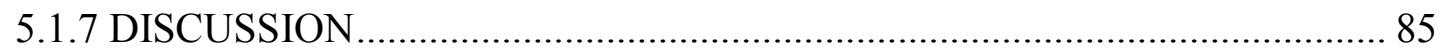

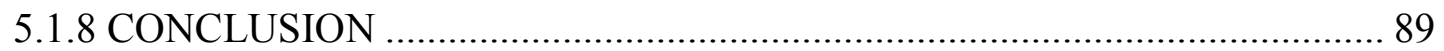

5.2 PHYSICAL AND SIMULATED PHANTOM STUDIES (HETEROGENEOUS

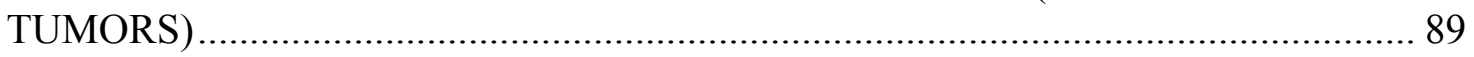

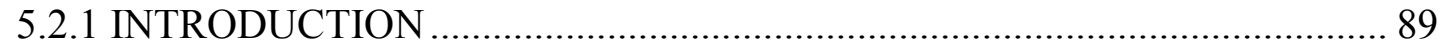

5.2.2 FV OF IRREGULAR SHAPED HETEROGENEOUS PHYSICAL TUMORS

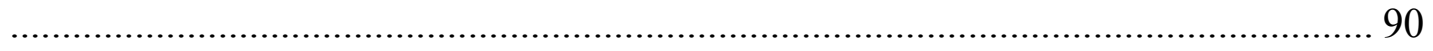

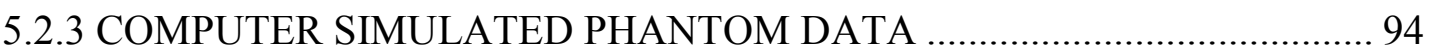

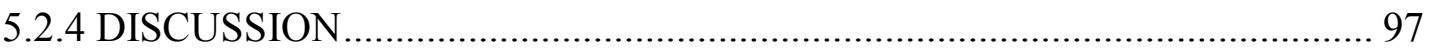

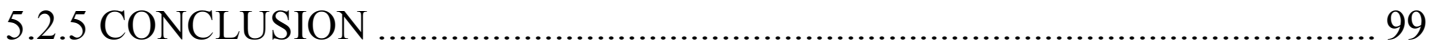

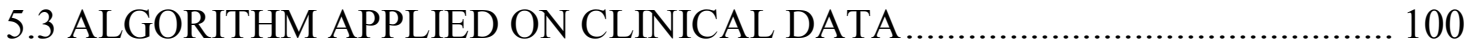

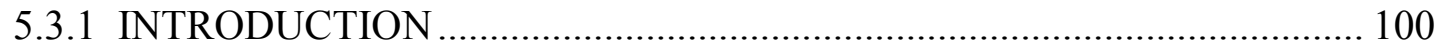

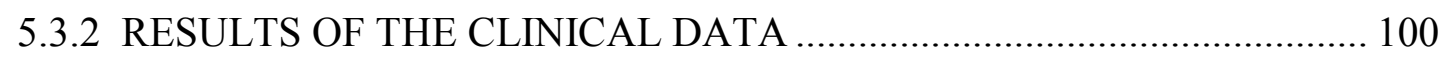

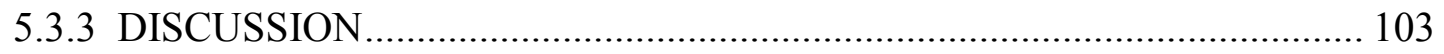

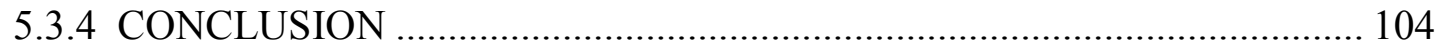

5.4 PHANTOM DATA WITH DIFFERENT RECONSTRUCTION PARAMETERS

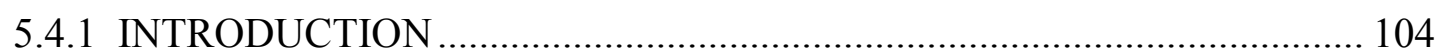

5.4.2 RESULTS OF DIFFERENT RECONSTRUCTION PARAMETER ........... 105

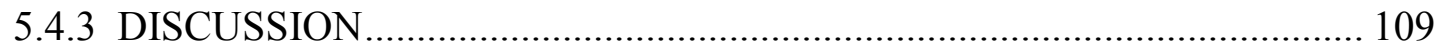

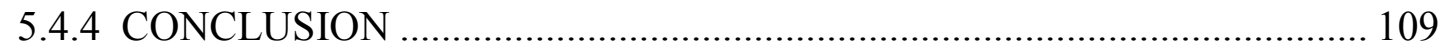

CHAPTER 6 Summary........................................................................................ 110

6.1 OVERALL DISCUSSION AND SIGNIFICANCE OF THE WORK ................ 110

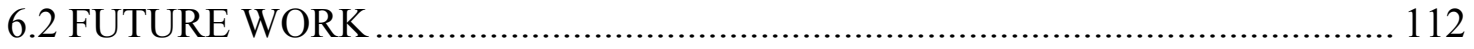

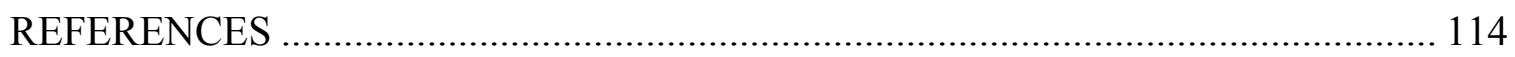

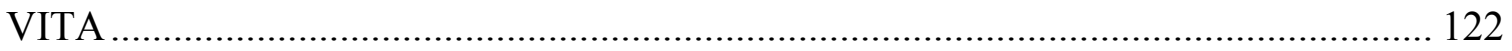




\section{LIST OF FIGURES}

FIGURE

PAGE

Figure 1.1 (left) a CT image of a patient with liver tumor, (right) PET/CT image of the same patient but in this image necrotic core within the tumor is visible. 2

Figure 1.2 we see the effect of partial volume effect (PVE) on an 2D object, where the size of the measured object (right) is larger and activity is smaller than the actual object (left) 4

Figure 1.3 Flow chart of the organization of the complete dissertation ......................... 7

Figure 1.4 Illustrated representation showing the particles injected inside a tumor.......... 8

Figure 1.5 Conceptual illustration of how the external radiation therapy works, where different colors represent different energy beams 10

Figure 1.6 Dose painting. Different colors represent different amount dose given in centigray (cGy). 10

Figure 3.1 The complete outline of the tumor quantification algorithm 18

Figure 3.3 The deconvolution applied on the line signal (solid line) from a $4 \mathrm{ml}$ tumor (a) and $16 \mathrm{ml}$ tumor (b) after 8 iterations. $D S$ (dotted line) is the segmented deconvolved signal. (a) The value of $D S$ increases from the center and approaches zero at the edge. (b) The value of $D S$ approaches zero at the center and the edge.

Figure 3.4 Complete block diagram of maximum intensity correction (MIC) algorithm. The output from the MIC is used as the stopping condition in the main IDTS algorithm.

Figure 3.5 The progression of HT algorithm after (a) iteration 1, (b) iteration 5 and (c) iteration 30 .

Figure 3.6 The progression of altered HT algorithm with at (a) iteration 1, (b) iteration 5 and (c) iteration 30.

Figure 3.7 (a) Original image with ROI. (b) The segmentation of the tumor fails with the application of the original HT algorithm. (c) The altered HT algorithm was able to successfully segment the tumors...... 35

Figure 4.1 Example of clincial data where the activity of the kidney is similar to the tumor (a) transverse slice (b) coronal slice (c) sagittal slice. 39 
Figure 4.2 Example of clincial data where the activity of the heart is similar to the tumor (a) transverse slice (b) coronal slice (c) sagittal slice.

Figure 4.3 Example of clincial data where the activity of the spine is similar to the tumor (a) transverse slice (b) coronal slice (c) sagittal slice.

Figure 4.4 Outline of the VOI generating algorithm (a) Original image (b) ROI on MIP in coronal view (c) ROI on MIP in sagittal view (d) ROI on MIP image in transverse view and (e) showing 3D rendering of the VOI (red). 42

Figure 4.5 Transverse view of slices of patient 1 where the VOI (red) only surround the region in the liver and avoids the kidney and spine.

Figure 4.6 Transverse view of slices of patient 2 where the VOI (red) only surround the region in the liver and avoids the kidney and spine 45

Figure 5.1 Three types of manually drawn ROI (a) The ROI is drawn on every slice close to the tumor. (c) The ROI is drawn on one slice and drawn bigger than the tumor. (e) The ROI drawn includes multiple tumors. (g) A very large ROI is drawn on one slice. (b, d, $f$ and $\mathrm{h}$ ) Result after segmentation, where in h, the segmentation completely fails. 49

Figure 5.2 The ratio of SG volume/True volume after HT algorithm for different SBR. 51

Figure 5.3 FV for different SBR by changing FWHM $(4 \mathrm{~mm}, 6 \mathrm{~mm}, 8 \mathrm{~mm}$ and $10 \mathrm{~mm})$. The FV in (a), (b) and (c) is for pixel size $5.46 \mathrm{~mm}$ and in (d), (e) and (f) pixel size is $3.64 \mathrm{~mm}$. The scan time is $5 \mathrm{~min}$ and matrix size is $5 \times 5$. Error bars correspond to standard deviation of the number of data sets $(\mathrm{n}=2)$. 53

Figure 5.4 RC for different SBR by changing FWHM (4 mm, $6 \mathrm{~mm}, 8 \mathrm{~mm}$ and $10 \mathrm{~mm})$. The RC in (a), (b) and (c) is for pixel size $5.46 \mathrm{~mm}$ and in (d), (e) and (f) pixel size is $3.64 \mathrm{~mm}$. The scan time is $5 \mathrm{~min}$ and matrix size is $5 \times 5$. Error bars correspond to

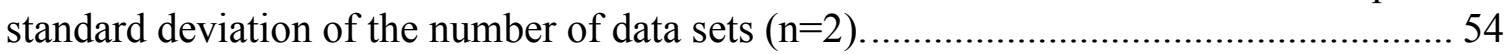

Figure 5.5 FV for different SBR by changing matrix size $(3 \times 3,5 \times 5$ and $7 \times 7)$. The FV in (a), (b) and (c) is for pixel size $5.46 \mathrm{~mm}$ and in (d), (e) and (f) pixel size is $3.64 \mathrm{~mm}$. The scan time is $5 \mathrm{~min}$ and FWHM is $6 \mathrm{~mm}$. Error bars correspond to standard deviation of the number of data sets $(n=2)$. 55

Figure 5.6 RC for different SBR by changing matrix size $(3 \times 3,5 \times 5$ and $7 \times 7)$. The $\mathrm{RC}$ in (a), (b) and (c) is for pixel size $5.46 \mathrm{~mm}$ and in (d), (e) and (f) pixel size is $3.64 \mathrm{~mm}$. The scan time is $5 \mathrm{~min}$ and FWHM is $6 \mathrm{~mm}$. Error bars correspond to standard deviation of the number of data sets $(n=2)$. 56 
Figure 5.7 FV for different SBR by changing scan time (1 min, $3 \mathrm{~min}$ and $5 \mathrm{~min}$ ). The FV in (a), (b) and (c) is for pixel size $5.46 \mathrm{~mm}$ and in (d), (e) and (f) pixel size is $3.64 \mathrm{~mm}$. The FWHM is kept at $6 \mathrm{~mm}$ and matrix size is $5 \times 5$. Error bars correspond to standard deviation of the number of data sets $(n=2)$. 57

Figure 5.8 $\mathrm{RC}$ for different SBR by changing scan time (1 $\mathrm{min}, 3 \mathrm{~min}$ and $5 \mathrm{~min}$ ). The $\mathrm{RC}$ in (a), (b) and (c) is for pixel size $5.46 \mathrm{~mm}$ and in (d), (e) and (f) pixel size is 3.64 $\mathrm{mm}$. The FWHM is kept at $6 \mathrm{~mm}$ and matrix size is $5 \times 5$. Error bars correspond to

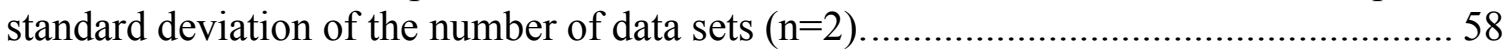
Figure 5.9 Contribution of different parameter in terms of effect values in measurement of (a) FV and (b) RC for different size tumors. 63

Figure 5.10 Contribution of different parameters in term of Effect values for measuring (a) FV and (b) RC considering true volume (TV) as one of the parameter. 76

Figure 5.11 Schematic of a phantom with an irregular shaped heterogeneous physic tumor (indicated by the red dotted circle). 90

Figure 5.12 Physical tumors with spherical cores of different activity concentrations to represent tumors with heterogeneous uptake distribution. (a) Three tumors with spherical cores, with different activity concentration between the tumors and their cores. The tumor

Figure 5.13 The percentage of volume from irregular shaped heterogeneous simulated tumors. (a) pixel size $5.46 \mathrm{~mm}$ and (b) pixel size $3.64 \mathrm{~mm}$. The FWHM is kept at $6 \mathrm{~mm}$ and matrix size is $5 \times 5$. Error bars correspond to the variation due to different image acquisition times or image noise ( $5 \mathrm{~min}, 3 \mathrm{~min}$ and $1 \mathrm{~min}$ ). 93

Figure 5.14 NCAT simulated phantom where a heterogeneous tumor is incorporated inside the liver, SUV of large tumor $(51 \mathrm{ml})$ is 6 and the SUV of the heterogeneous section $(6.8 \mathrm{ml})$ of the tumor is 9 . The SUV of the liver and kidney is kept at $1 . .$. 95

Figure 5.15 The results from three methods of segmentation on 5 out of total 10 patient data sets: column 1 shows the original image and column 2 shows the output of the IDTS algorithm, and columns 2 and 3 give results of the application of T50 and T60 segmentation methods, respectively. 101

Figure 5.16 Results of FV estimation with reconstruction parameter 8 subset $\times 1$ iterations, for different signal to background ratio (SBR) (a) 2.74 (b) 4.4 and (c) 7.3. The FWHM of the point spread function was kept at $6 \mathrm{~mm}$ and matrix size $5 \times 5$ and the acquisition time was 5 min. 105

Figure 5.17 Results of FV estimation with reconstruction parameter 8 subset $\times 2$ iterations, for different signal to background ratio (SBR) (a) 2.74 (b) 4.4 and (c) 7.3. The 
FWHM of the point spread function was kept at $6 \mathrm{~mm}$ and matrix size $5 \times 5$ and the acquisition time was 5 min. 106

Figure 5.18 Results of FV estimation with reconstruction parameter 8 subset $\times 8$ iterations, for different signal to background ratio (SBR) (a) 2.74 (b) 4.4 and (c) 7.3. The FWHM of the point spread function was kept at $6 \mathrm{~mm}$ and matrix size $5 \times 5$ and the acquisition time was 5 min. 107

Figure 5.19 Results of FV estimation with reconstruction parameter 18 subset $\times 4$ iterations, for different signal to background ratio (SBR) (a) 2.74 (b) 4.4 and (c) 7.3. The FWHM of the point spread function was kept at $6 \mathrm{~mm}$ and matrix size $5 \times 5$ and the acquisition time was 5 min. 107

Figure 5.20 FV and mAC estimation after of altered histogram thresholding (HT) algorithm. 


\section{LIST OF TABLES}

TABLE

PAGE

Table 5.1 Parameters (factors) and their values (levels) used in the factorial analysis. All factor levels are treated as nominal data.

Table 5.2 Effect values of each of the parameters (factors) and their corresponding interaction for the response variable FV. (TV = tumor volume, IS = image size, AT = aqusition time, $\mathrm{SBR}=$ signal to background ratio, $\mathrm{MS}=$ matrix size and $\mathrm{FWHM}=$ full width half maximum). 77

Table 5.3 Effect values of each of the parameters (factors) and their corresponding interaction for the response variable RC. (TV = tumor volume, IS = image size, AT = aqusition time, $\mathrm{SBR}=$ signal to background ratio, $\mathrm{MS}=$ matrix size and $\mathrm{FWHM}=$ full width half maximum). 81

Table 5.4. Specifications of the irregular shaped heterogeneous physical tumor phantom experiments. 91

Table 5.5. Percentage of true volume (\% of True Volume) and classification error (CE) of the algorithm in segmenting NCAT simulated heterogeneous tumors. The algorithm is applied by changing FWHM from $4 \mathrm{~mm}, 6 \mathrm{~mm}, 8 \mathrm{~mm}$ to $10 \mathrm{~mm}$. The matrix size of PSF is kept at $5 \times 5$.

Table 5.6 Results showing percentage of volume error and percentage of mean activity concentration (mAC) error of IDTS algorithm, T50 and T60 when compared to manual segmentation 102 


\section{LIST OF SYMBOLS}

\begin{tabular}{|c|c|}
\hline$x$ & $\mathrm{X}$-directional axis \\
\hline$y$ & Y-directional axis \\
\hline$z$ & Z-directional axis \\
\hline$X$ & Size in X-direction \\
\hline$Y$ & Size in Y-direction \\
\hline$Z$ & Size in Z-direction \\
\hline$B K$ & Background \\
\hline$S G$ & Segment \\
\hline$k$ & Number of iteration \\
\hline$T$ & Bin value \\
\hline$Q$ & Bin number \\
\hline$P$ & Total number of bin \\
\hline$H_{B K}(T)$ & Histogram of background \\
\hline$H_{S G}(T)$ & Histogram of segment \\
\hline$I(x, y, z)$ & Image \\
\hline$R O I$ & Region of interest \\
\hline$T_{z}$ & Threshold \\
\hline$B I$ & Binary image \\
\hline$T U$ & Binary mask \\
\hline$D E$ & Deconvolved image \\
\hline$D S$ & Segment of the deconvolved image \\
\hline$V O$ & Total number of voxels segment \\
\hline$M V$ & Value of maximum intensity of tumor \\
\hline$j$ & Number of iteration \\
\hline$B R$ & Blur pixels \\
\hline$M B V$ & Mean value of blur pixels \\
\hline$M B$ & Mean background \\
\hline
\end{tabular}




$D L$
$I_{\text {coronal }}$
$I_{\text {sagittal }}$
$I_{\text {transversel }}$
$R O I_{\text {coronal }}$
$R O I_{\text {coronal }}$
$R O I_{\text {coronal }}$

Pixels in the dilated image

Maximum intensity projected image in coronal view

Maximum intensity projected image in sagittal view

Maximum intensity projected image in transverse view

Region of interest drawn on $I_{\text {coronal }}$

Region of interest drawn on $I_{\text {sagittal }}$

Region of interest drawn on $I_{\text {transversel }}$ 


\section{LIST OF ABBREVIATIONS AND ACRONYMS}

\begin{tabular}{|c|c|}
\hline PET & Positron emission tomography \\
\hline $\mathrm{CT}$ & Computed tomography \\
\hline MRI & Magnetic resonance imaging \\
\hline $\mathrm{HCC}$ & Hepatocellular carcinoma \\
\hline SIRT & Selective internal radiation therapy \\
\hline $3 \mathrm{D}$ & Three dimensional \\
\hline $2 \mathrm{D}$ & Two dimensional \\
\hline BD-CRT & Three dimensional conformal radiation therapy \\
\hline IMRT & Image modulated radiation therapy \\
\hline IGRT & Image guided radiation therapy \\
\hline SUV & Standard uptake value \\
\hline ROI & Region of interest \\
\hline $\mathrm{AC}$ & Activity concentration \\
\hline $\mathrm{mAC}$ & Mean activity concentration \\
\hline FV & Functional volume \\
\hline TLG & Total lesion glycolysis \\
\hline Mbq & Megabacquerel \\
\hline Gbq & Gigabacquerel \\
\hline gm & gram \\
\hline PSF & Point spread function \\
\hline FWHM & Full width half maximum \\
\hline IDTS & $\begin{array}{l}\text { Iterative deconvolution thresholding } \\
\text { segmentation }\end{array}$ \\
\hline NCAT & NURBS based Cardio-torso \\
\hline VOI & Volume of interest \\
\hline BSA & Body surface area \\
\hline cGy & centigray \\
\hline HT & Histogram thresholding \\
\hline
\end{tabular}




\begin{tabular}{|c|c|}
\hline PVE & Partial volume effect \\
\hline MHT & Modified histogram thresholding \\
\hline MIC & Maximum intensity correction \\
\hline $\mathrm{mm}$ & millimeter \\
\hline $\min$ & minute \\
\hline SBR & Signal to background ratio \\
\hline MIP & Maximum intensity projected image \\
\hline SD & Standard deviation \\
\hline $\mathrm{RC}$ & Recovery coefficient \\
\hline $\mathrm{ml}$ & milliliter \\
\hline 1 & liter \\
\hline $\mathrm{CE}$ & Classification error \\
\hline PCE & Positive classification error \\
\hline NCE & Negative classification error \\
\hline SUVmean & Mean standard uptake value \\
\hline SUVmax & Maximum standard uptake value \\
\hline SUVpeak & Peak standard uptake value \\
\hline GUI & Graphical user interface \\
\hline${ }^{18} \mathrm{~F}$ & Fluoride-18 \\
\hline FDG & Fluorodeoxyglucose \\
\hline TV & True volume \\
\hline
\end{tabular}




\section{CHAPTER 1}

\section{Introduction}

Liver cancer is one of the major causes of death due to cancer; it is the fifth most common among men (13,980 deaths) and ninth most common among women (6,570 deaths), with 21,370 and 7,350 new cases annually in men and women, respectively [1]. Radiation therapy is one of the promising treatment methods for patients with unresectable ${ }^{1}$ hepatocellular carcinoma $(\mathrm{HCC})^{2}$. There are two main types of radiation therapy. The first is internal radiation therapy, an example of whichis selective internal radiation therapy (SIRT). The second is external radiation therapy, and examples of it are three-dimensional conformal radiation therapy (3D-CRT), image modulated radiation therapy (IMRT) and image guided radiation therapy (IGRT). The explanation of the different radiation therapies will be provided in the later section.

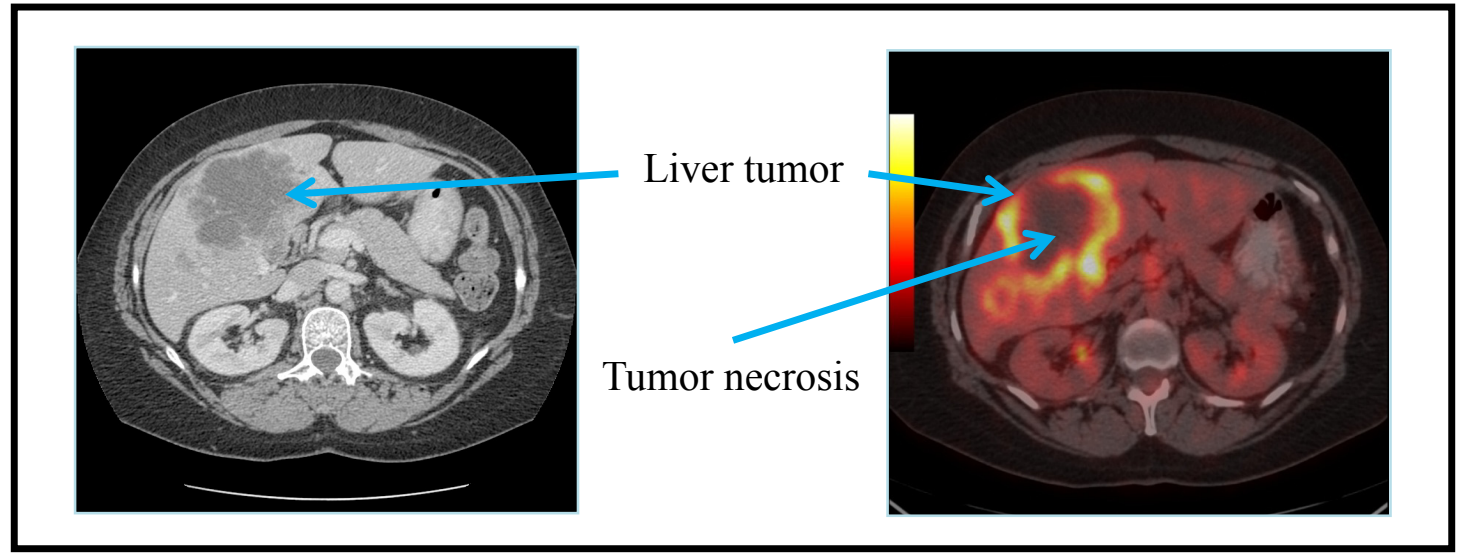

\footnotetext{
${ }^{1}$ Tumors which cannot be surgically removed for variety of reasons which include size, location and the stage of the tumors

${ }^{2}$ Most common type of liver cancer
} 
Figure 1.1 (left) a CT image of a patient with liver tumor, (right) PET/CT image of the same patient but in this image necrotic core within the tumor is visible.

For all of the above mentioned radiation therapies, the most important starting point is determining the accurate tumor volume and its contour ${ }^{3}$. Tumor volume is used to determine the dose for a particular therapy. Most of the radiation therapies use computed tomography (CT) for calculating tumor volume because of the low cost compared to MRI and PET. CT and MRI give only anatomical information of a tumor, whereas positron emission tomography (PET) provides functional information. By providing functional information, PET is able to distinguish between tissues which are metabolically active and which are necrotic. In Figure 1.1 (right) we can see a PET/CT image with a liver tumor having necrotic core but in Figure 1.1 (left) CT image shows liver tumor as a large uniform mass without the necrotic core. Using anatomical information provided by the CT and MRI can sometimes lead to error in the dose estimation.

Utilizing tumor volume for dose measurement is just one application, another important use of tumor volume is for prognostic value, i.e. for predicting the progression or outcome of a tumor upon a treatment [2-9]. Keeping track of the tumor volume over the lifetime of the treatment can lead to a better understanding of how effective a given treatment is for a given tumor type.

Another important aspect of tumor quantification is estimating metabolic activity ${ }^{4}$ of the tumor from PET. If a tumor shows more metabolic activity the chances of it being malignant is much higher.

\footnotetext{
${ }^{3}$ Location and the margins of a tumor

${ }^{4}$ Amount of glucose uptake by a tumor
} 
For a better comparison of metabolic activity between different patients or the same patient at different times, the term standard uptake value (SUV) is widely used. SUV is the ratio of the mean activity in a region of interest (ROI) times the body weight to the injected dose (Equation 1.1). The mean activity in a ROI can also be called the mean activity concentration (mAC) of tumors if the ROI includes only tumors. The ROI can include both tumors and organs depending on what the SUV is being measured for. The quantity $\mathrm{mAC}^{5}$ is dependent on the functional volume ${ }^{6}(\mathrm{FV})$. Another term that is used as a prognostic value is called total lesion glycolysis (TLG), which is the product of FV and SUV [10-13]. From this definition we can see that the accuracy of TLG is also dependent on tumor FV.

$$
S U V=\frac{\text { Mean ROI concentration }(\mathrm{Mbq} / \mathrm{gm})}{\text { Injected dose }(\mathrm{Mbq}) / \text { Body weight }(\mathrm{gm})}
$$

One limitation of PET is that the resolution of a PET image is very low compared to CT and MRI images. The low resolution of PET is due to the physics of the imaging modality (positron range and scatter) and also due to the number of detector crystals on a camera's gantry. The PET images are also noisier compared to CT and MRI images; this noise is due to scatter and low sensitivity of PET. There are many methods by which the noise in a PET image can be reduced; one is by using the right kind of filter, but too much filtering can also reduce the contrast; second is to use cameras having time of flight (TOF) capability, even though it is a very promising method it is expensive and provides limited gain compared to a standard PET camera.

Another limitation of PET is that PET images suffer from partial volume effect (PVE). PVE is equivalent to the convolution of the actual image with the camera's point spread function

\footnotetext{
${ }^{5}$ Mean activity is the ratio of sum of all the intensity values of pixels segmented as tumor times the number of pixels segmented

${ }^{6}$ It's the metabolically active volume measured from PET
} 
(PSF). The spatial resolution of the camera is equal to the full width half maximum (FWHM) of the Gaussian PSF. The PSF is not spatially uniform, so retrieving the original image is an illposed problem [14]. In Figure 1.2 we observe that PVE makes the margins of the actual object blurry, and this blur is what makes it difficult to quantify the tumor FV.

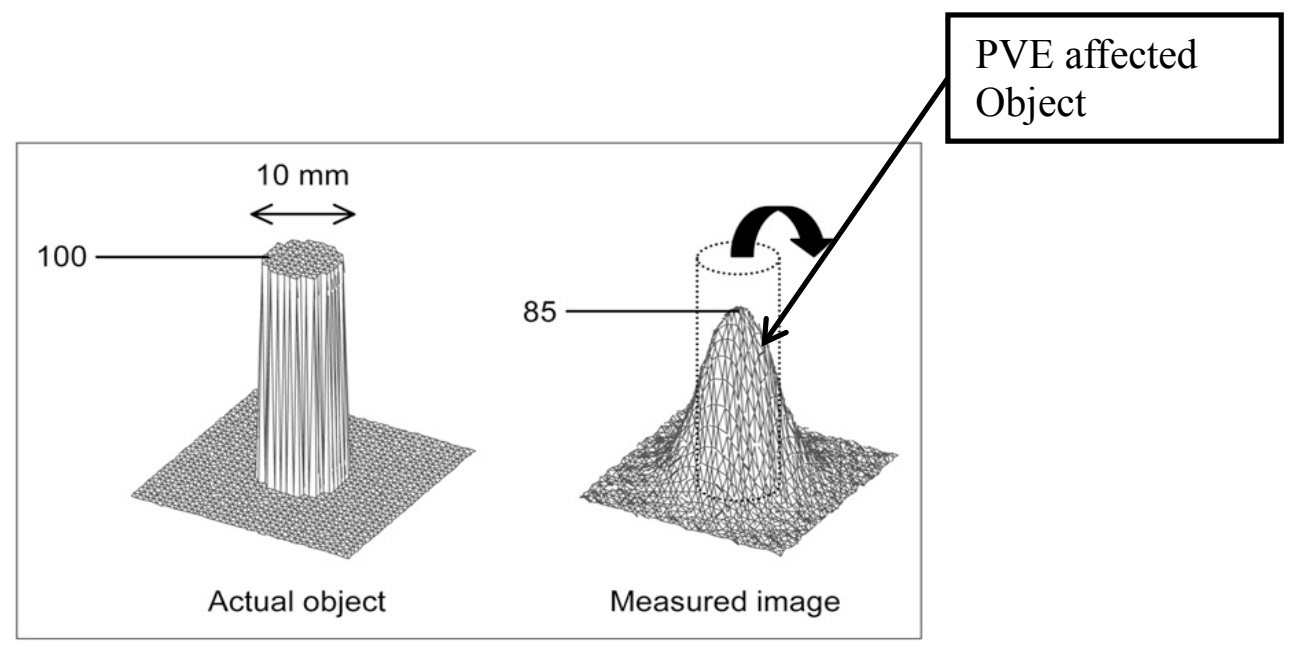

Figure 1.2 we see the effect of partial volume effect (PVE) on an 2D object, where the size of the measured object (right) is larger and activity is smaller than the actual object (left) (image modified from Soret et al 2007) [15].

To date, manual segmentation is still considered the gold standard for segmenting a tumor. The manual segmentation of tumors in PET images is time consuming, which can lead to attrition on the part of radiologist, affecting the result. Additionally, the results from manual segmentation are very hard to reproduce making it difficult to use if large sets of data are required to be processed in the clinic. The importance of the tumor FV and the impracticality of manual segmentation are two of the motivations behind developing an automatic tumor segmentation and quantification algorithm for PET images.

Currently, there is no standard protocol in which PET images are reconstructed, as they vary with different manufacturers. Each manufacturer of a PET camera uses slightly different 
geometries, detectors size and reconstruction algorithms; this makes it imperative to develop an algorithm which does not need to be optimized for a particular camera. I have developed iterative deconvolution thresholding segmentation (IDTS) algorithm which is the combination of the histogram thresholding and Van-Cittert deconvolution methods. The algorithm is unique as it segments; measures FV, and also measures PVE corrected mAC. I have formulated the stopping conditions within the algorithm in such a way that the algorithm does not need to be optimized for a particular camera. This feature will enable the algorithm to be useful in analysis of multicenter PET data. The algorithm, as an initiation step, requires user input in generating a volume of interest (VOI), after which the algorithm is automatic. Herein, the IDTS algorithm is tested on a physical tumor phantom with homogeneous and heterogeneous activity, simulated phantom data-NCAT phantom with added Gaussian blur and noise to mimic real data and clinical data which is validated with comparison to manual segmentation by a radiologist.

\subsection{ORGANISATION OF THE DISSERTATION}

The dissertation is organized as follows and the flow of the dissertation is given in Figure 1.3:

Chapter: 1 gives Introduction and the description of different radiation therapies, SIRT, 3DCRT, IMRT and IGRT. The chapter also gives information on how the tumor FV is used to calculate prescribed dose.

Chapter: 2 provides background on different segmentation and FV estimation algorithms and how they vary from each other. And also gives information about different PVE correction algorithms which are used to calculate corrected activity concentration. 
Chapter: 3 mathematically describes the iterative deconvolution thresholding segmentation (IDTS) algorithm. The chapter also contains information about alterations made to the IDTS algorithm so that it can be successfully applied on clinical data.

Chapter: 4 describes the algorithm to generate volume of interest (VOI) from user drawn ROI.

Chapter: 5 provides results of estimated $\mathrm{FV}$ and $\mathrm{mAC}$ from physical phantoms with homogeneous activity profile and on tumors with heterogeneous activity profile, generated through physical phantom and simulated phantom studies. The chapter also provides the results of the impact of tumor FV and $\mathrm{mAC}$ with the change in reconstruction algorithm parameters. The chapter provides result of the IDTS algorithm when applied on clinical data. The result from the IDTS algorithm and fixed percentage thresholding are compared to the manual segmentation (gold standard). In this chapter we apply the altered IDTS on phantom data and compare the variation in the results to the IDTS algorithm.

Chapter: 6 provides general discussion and significance of the work and also gives an outline of possible future work. 


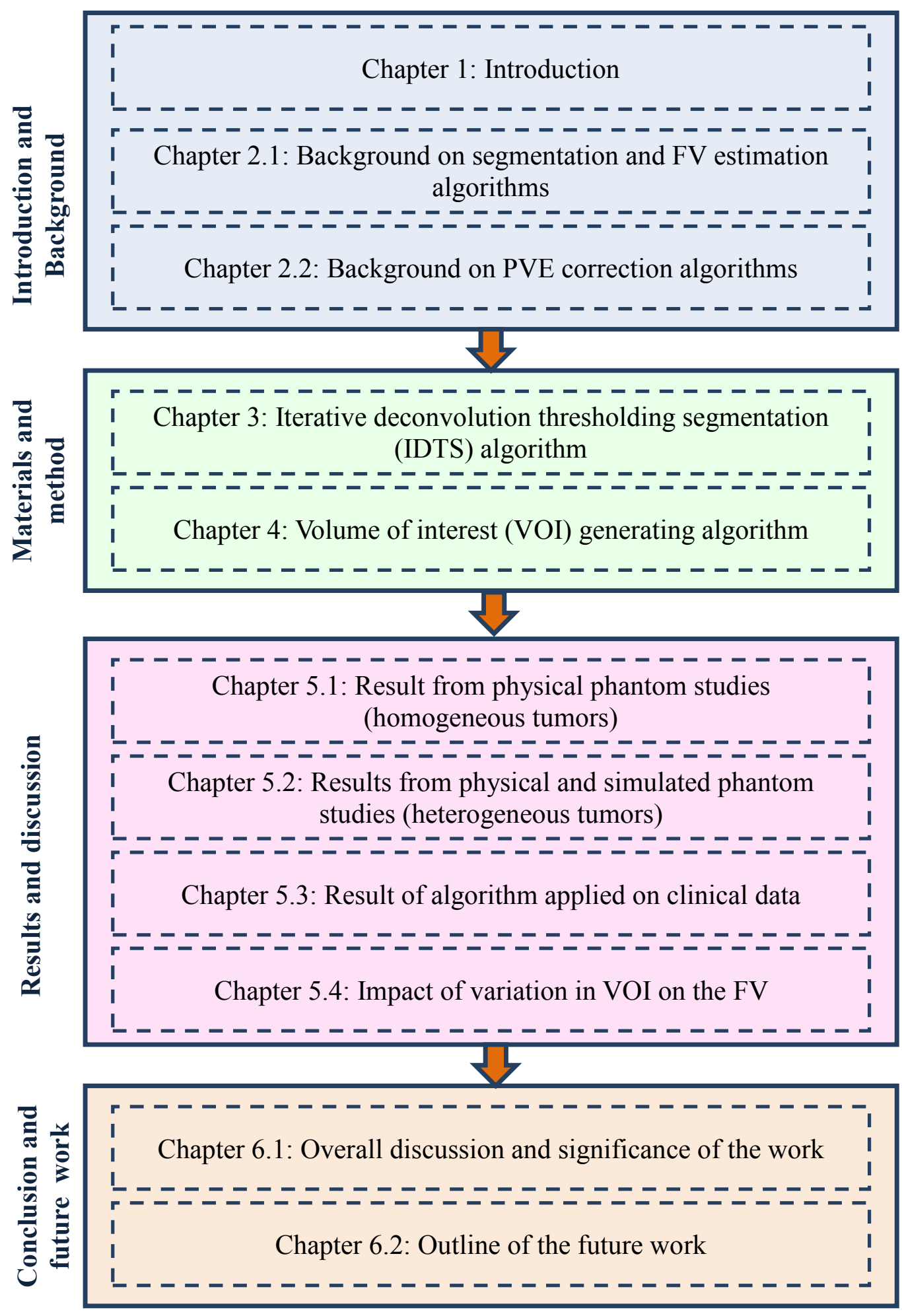

Figure 1.3 Flow chart of the organization of the complete dissertation 


\subsection{RADIATION THERAPY}

\subsubsection{INTRODUCTION}

Radiation therapy is a potential treatment for patients who have unrespectable hepatocellular carcinoma. There are two types of radiation therapy: external radiation therapy and internal radiotherapy. This chapter provides a brief description of the different radiation therapy methods and how dose is calculated.

\subsubsection{SELECTIVE INTERNAL RADIATION THERAPY}

In selective internal radiation therapy (SIRT) a micro-particle is tagged with a beta emitting radioisotope (e.g. Yattrium-90). The SIRT therapy is also termed as radioemboization therapy. The SIRT treatment kills the tumor in two ways: 1) micro-particles are lodged and get stuck in the tumor where they stop the supply of oxygen and thereby starve the tumor cells, 2) the radioisotope which is tagged gives out beta radiation thereby killing the tumor cells. The range of the beta emitter is not high so the normal tissues are spared from radiation. Figure 1.4 shows an illustration of how the particle gets lodged into the tumor through the arteries.

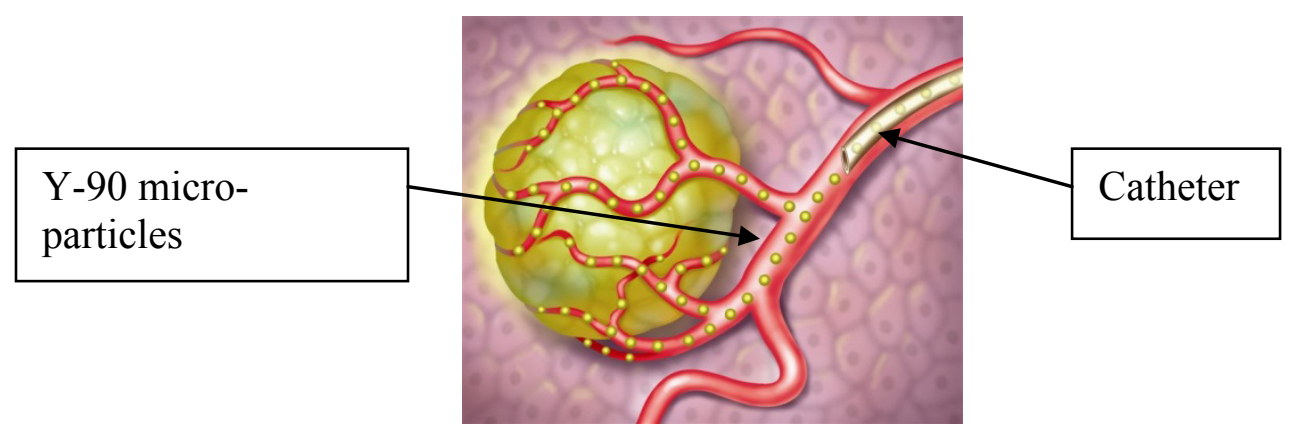

Figure 1.4 Illustrated representation showing the particles injected inside a tumor.

There are two types of particle mostly used in the clinics Sir-spheres and TheraSphere. Sir-spheres are micro resin particles and TheraSpheres are glass particles. This particle are 
tagged with yattrium-90 (Y-90) radioisotope and injected through the hepatic artery. There have been many literature publications comparing the performance of the two spheres with equal efficacy [16-19]. The dose for SIRT treatment has been mostly estimated using computed tomography (CT) images [20-23]. PET has also been recently used for estimating dose and where liver tumor volume and liver tumor contour are combined to estimate dose [24]. The formulation of the dose calculation is given in the Equation 1.2 below

$$
\operatorname{Dose}(\mathrm{GBq})=\mathrm{BSA}-0.2 \frac{\text { Volume }_{\text {tumor }}}{\text { Volume }_{\text {tumor }}-\text { Volume }_{\text {liver }}}
$$

where BSA is body surface area. The role of PET in the SIRT treatment has been steadily increasing, not just for treatment planning, but also for prognostic evaluation [25-27]. Both tumor volume and standard uptake value are used for prognostic evaluation $[22,25,26]$.

\subsubsection{EXTERNAL RADIATION THERAPY}

There are many forms external radiation therapy, including the ones called three dimensional conformal radiation therapy (3D-CRT). In this therapy a topographic image is take like computed tomography (CT), magnetic resonance imaging (MRI) or positron emission tomography (PET) to construct a 3D stereoscopic image and through computer software the radiation beam is conformed around the tumor. Figure 1.5 gives an example of how an external beam is applied on tumors in the abdomen. 


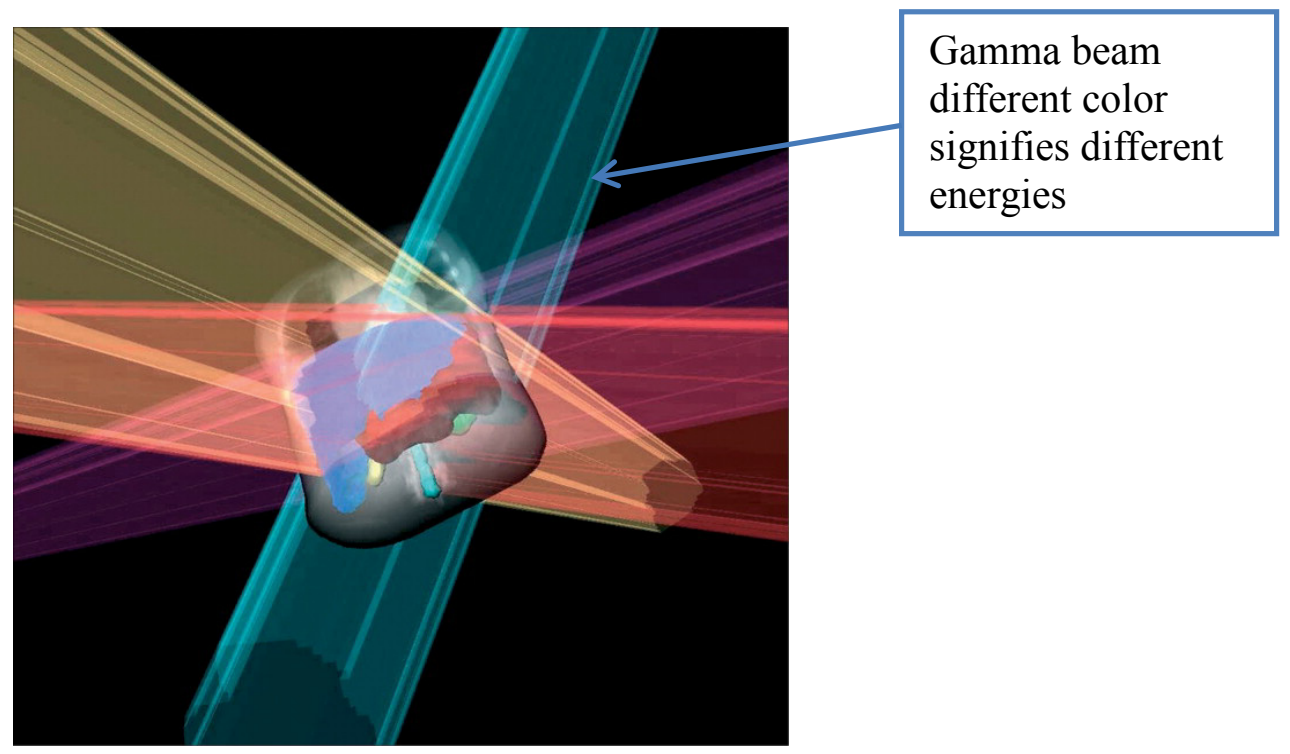

Figure 1.5 Conceptual illustration of how the external radiation therapy works, where different colors represent different energy beams (Image modified from Hartgrink et al) [28]

The 3D-CRT only provides uniform intensity over the volume and the next evolution of the treatment is called intensity modulated radiation therapy (IMRT). The IMRT differs from 3D-CRT such that IMRT uses a modulated radiation beam which can apply precise dose based on the tissue type. IMRT treatment is mostly applied on head and neck tumors and the dose is calculated using CT images. The dose applied is calculated by software based on the image; this concept is called dose painting (Figure 1.6).

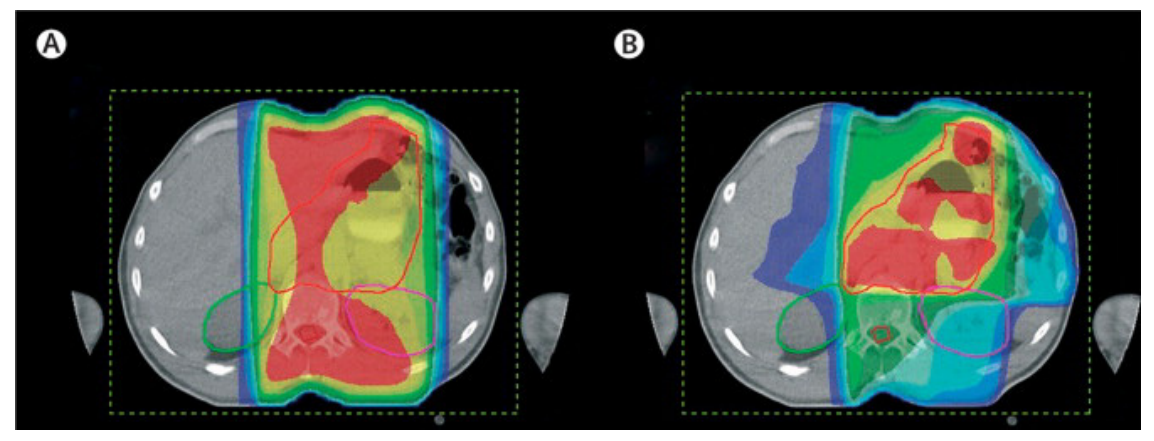

Figure 1.6 Dose painting. Different colors represent different amount dose given in centigray (cGy). (Image modified from Hartgrink et al) [28]. 
Recently PET images are used for head and neck tumor contour volume since CT images cannot provide information if the region is necrotic or hypoxic. If the regions within the tumor are hypoxic it requires higher dose than the rest of the tumor $[29,30]$.

The next evolution of the external radiation is called image guided radiation therapy (IGRT) where the treatment is adapted based on the movement of the tumor. More than IMRT, IGRT provides better treatment protocol when applied in the liver as the tumors in liver move due to breathing. 


\title{
CHAPTER 2
}

\author{
Background
}

\subsection{FUNCTIONAL VOLUME ESTIMATION ALGORITHM}

\subsubsection{INTRODUCTION}

The functional volume (FV) estimation starts with tumor segmentation. There are many algorithms which find FV directly or apply segmentation and FV estimation in two steps [31, 32]. The major kind of segmentation algorithms are adaptive thresholding, active contouring (also termed as snakes), gradient based segmentation, clustering algorithm and fuzzy logic based segmentation.

\subsubsection{ADAPTIVE THRESHOLDING}

Adaptive thresholding uses features from the image by fitting it to the actual volume. The parameters are derived from the curve and used for segmentation [33-39]. The adaptive threshold needs to be optimized for each camera as the fitting curve for one camera does not apply to other cameras. The features are mostly found by doing phantom studies, which makes it difficult to apply if any of the features changed from the phantom studies. It is difficult to fit a curve and find parameters for all cases as the phantom data is very simple and cannot correlate well with a clinical image.

\subsubsection{ACTIVE CONTOUR SEGMENTAITON}

The active contour method (also termed as snakes) finds the point of low energy around the image [31, 40, 41]. The active contour algorithm requires a pre-stage, usually filtering or thresholding, as the performance of the active contour method is very limited in PET images. 
This algorithm has very limited scope as it requires tumors that are well separated. Li et al. used a region growing algorithm to segment all the tumors and then used an active contour method to find the FV [31]. The algorithm requires input parameters which must be optimized by performing phantom studies.

\subsubsection{GRADIENT BASED SEGMENTATION}

Gradient based segmentation uses a gradient or edge detection of tumor boundaries to determine the tumor volume. Geets et al. used a watershed algorithm for segmenting tumors. The algorithm is applied in two steps where the deconvolution is applied to make the boundaries sharper. In PET images the boundaries of the tumor are very blurry and require some enhancement. They tested the algorithm with phantom data with spherical tumors but only using one signal to background ratio. The algorithm performed better in the clinical images but they are difficult to validate.

\subsubsection{CLUSTERING ALGORITHM}

The clustering algorithm is the machine learning algorithm which iteratively isolates the tumor from background. The algorithm uses fuzzy C-mean clustering which segment the pixels based on their location with respect to each other in order to remove the blur. A wavelet transform is used so that the algorithm could converge to the right volume [42]. Belhassen et al. used NCAT simulated phantom only to validate their algorithm and did not perform any physical phantom studies. The algorithm was able to segment tumors with heterogeneous activity profile [42]. Yang et al. also tested their algorithm on simulated phantom data and were able to segment heterogeneous tumors as well as the heterogeneity within the tumor [43]. 


\subsubsection{FUZZY BASED SEGMENTATION}

Fuzzy based segmentation uses fuzzy logic where the stochastic statistics are used to determine if a pixel belongs to the tumor or background [44]. Hatt et al. have developed a fuzzy logic based Bayesian segmentation algorithm. The algorithm is computationally expansive as it has to determine for each pixels if it belongs to background or tumor. The algorithm was tested on physical phantom data and also on simulated phantom data. The algorithm failed to segment tumors less than $1 \mathrm{ml}$ in volume. The algorithm was later modified to make it able to segment heterogeneous region within the tumor [45]. 


\subsection{PVE CORRECTION ALGORITHM}

\subsubsection{INTRODUCTION}

Partial volume effect (PVE) is the major hurdle in the quantification of the tumors activity. The activity of the tumor is used to determine if the tumor is malignant or benign. The partial volume effect can make malignant tumor look benign [15]. There have been many methods of PVE correction but not all methods are applied for tumor quantification. One of the major applications of PVE correction is in brain studies [46]. The brain is a compact organ where activity from the desired sites spill out into neighboring regions. Algorithms used for PVE correction in the brain are beyond the scope of the research. The PVE correction algorithm changes from its application. PVE correction can be applied to quantify tumors or it can be applied to measure the volume of a compartment in kinetic studies [47]. There are two major methods of PVE correction; one uses anatomical data like computed tomography (CT) and magnetic resonance imaging (MRI) and another method uses deconvolution to correct for PVE.

\subsubsection{ANATOMICAL DATA BASED PARTIAL VOLUME EFFECT CORRECTION}

In this method the anatomical information provides the margin around the tumor and by blurring with the resolution of the camera and then subtracting and dividing with PET data we get the PVE corrected activity [48]. The problem with this method is that it requires co-

registration of anatomical data with the PET data for the PVE correction to be successful. This can be challenging if the patient moved during any of the scans. 


\subsubsection{DECONVOLUTION BASED PARTIAL VOLUME EFFECT CORRECTION}

The deconvolution method involves deconvolving the image with the point spread function (PSF) of the camera. Finding the right value of the PSF can be challenging as the PSF is not spatially uniform. Using a single value PSF in the deconvolution algorithm is not optimal for PVE correction. Moreover the deconvolution process adds noise within the image, and this noise can be controlled by adding a regularization term to the deconvolution algorithm [49]. Kirov et al. used the deconvolution method with regularization. The PSF they employed was not the cameras PSF but was custom made for their algorithm [49]. The PVE correction method only corrects the maximum intensity pixel and the mean value is not corrected. Teo et al. used VanCittert deconvolution but employed a slightly larger PSF function than the camera's [50]. On the other hand, Barbee et al. used spatially varying PSF which was acquired through phantom studies [14]. Boussion et al. used the Lucy-Richardson deconvolution method with wavelet transform [51]. They performed PVE correction on the entire image rather than on a single object. 


\section{CHAPTER 3}

Tumor Segmentation and Quantification Algorithm

\subsection{INTRODUCTION}

The whole tumor quantification algorithm is divided into four parts. The first part of the algorithm is called histogram thresholding (HT) where the tumor and the blur around the margins (due to PVE) of the tumor are segmented. The blur is included in order to measure the total activity of the tumor, since part of the activity from the tumor spills out to the background. The second part is called iterative deconvolution thresholding segmentation (IDTS), where the output from the HT algorithm is inputted and the blur around the tumor is segmented to derive FV. The third part of the algorithm is the stopping conditions; these stopping conditions enable the algorithm to be independent of the camera's PSF. The fourth and final part of the algorithm is called maximum intensity correction (MIC). An outline of the complete algorithm is shown in Figure 3.1. The second part of the method, the IDTS algorithm, utilizes a combination of deconvolution and the modified histogram thresholding (MHT) algorithm iteratively until the stopping condition is reached. The output of the IDTS algorithm is checked for volume. If the volume is $<1.8 \mathrm{ml}$ it is subjected to maximum intensity correction (MIC), which is shown in more detail in Figure 3.4 and will be discussed in a later section. The FV derived from the IDTS algorithm and the total activity from the HT algorithm is used in estimating the AC of the tumor. 


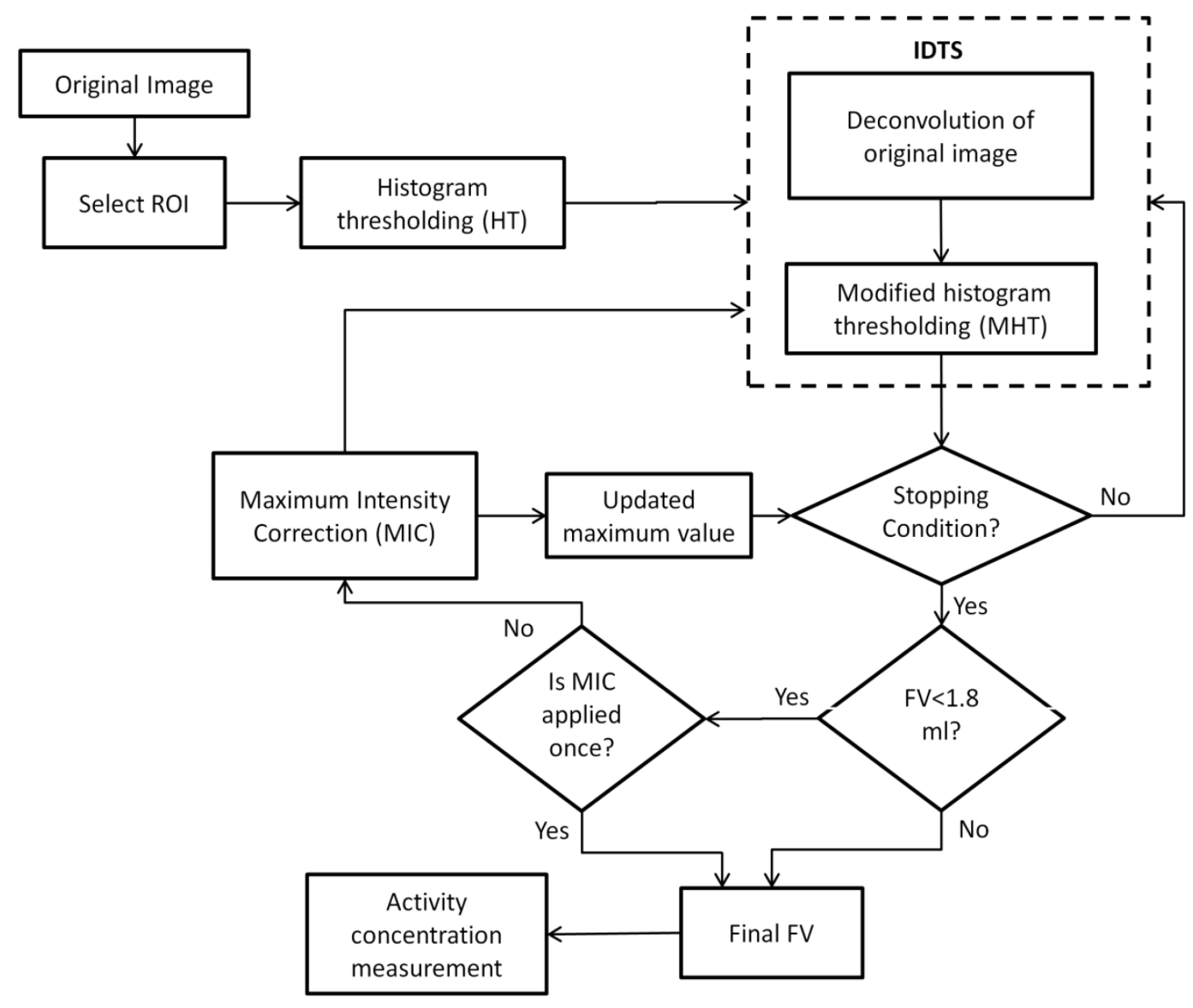

Figure 3.1 The complete outline of the tumor quantification algorithm.

The algorithm described in this paper was applied in two dimensions (2D) on each slice individually over the entire volume. Experimentally it was observed that the algorithm failed to segment small tumors $(<1 \mathrm{ml})$ when applied in three dimensions (3D). To avoid segmentation of local maxima due to the application of the algorithm in 2D, it was once applied on the transaxial plane and once on the sagittal plane and the intersection of the two volumes was considered as the FV. The variables considered in the paper are 3D functions of coordinates $(x, y, z)$; however, since operations are performed slice by slice, they will be written as $2 \mathrm{D}$ functions of $(x, y)$ with a subscript $z$ parameterizing the slice coordinate. The above formalism is correct for transaxial 
operations; for sagittal, $x$ is substituted for $z$ to obtain 2D functions of coordinates $(z, y)$ at different $x$ slices.

\subsection{HISTOGRAM THRESHOLDING ALGORITHM}

The PET image does not produce a bipeak (bimodal) histogram because of the huge intensity variation between tumor and background. This makes it impossible to find the point of maximum variance for the identification of optimal threshold, which would segment the tumor from the background. To overcome this limitation, a HT algorithm was developed which iteratively finds the optimal threshold. In Figure 3.2, the flow chart and different stages of the HT algorithm are shown. The HT algorithm is initialized by inputting the manually drawn ROI and ROI subtracted image called background $(B K)$. The HT algorithm gives out the segmented tumor $(S G)$ after $k$ iterations, and the value of $k=\{0,1,2, . ., k\}$. The HT algorithm works by iteratively finding the point of intersection between the histograms of background $H_{B K}^{k}(T)$ and the segment $H_{S G}^{k}(T) . T$ is the bin value, where $Q=\{0,1, . ., P\}$ is the bin number and $P$ is the total number of bins. The value of each bin is given by $T(Q)=(\max (I(x, y, z)) / P) \times Q$, where ' $\times$ ' is the multiplication operator. In both the histograms, the initial 10 bins are discarded since they primarily contain the pixels outside the phantom (or outside the body for clinical images), where the intensity is as much as 100 times lower than the intensity in the phantom. The histogram was divided into 256 bins, since increasing the number of bins made the histogram noisy and reducing the bin number below 256 can lead to sampling error where a wide range of intensities can be assigned to a single bin. For the initial segment in the HT algorithm the observer has to draw a 3D ROI around a tumor (Figure 3.2.b) in image $I(x, y, z)$ (Figure 3.2.a), where $x, y$ and $z$ indicate the $3 \mathrm{D}$ coordinate space and $x=\{1,2, . ., X\}, y=\{1,2, . ., Y\}$ and $z=\{1,2, . ., Z\}$ indicate the size of each dimension. 


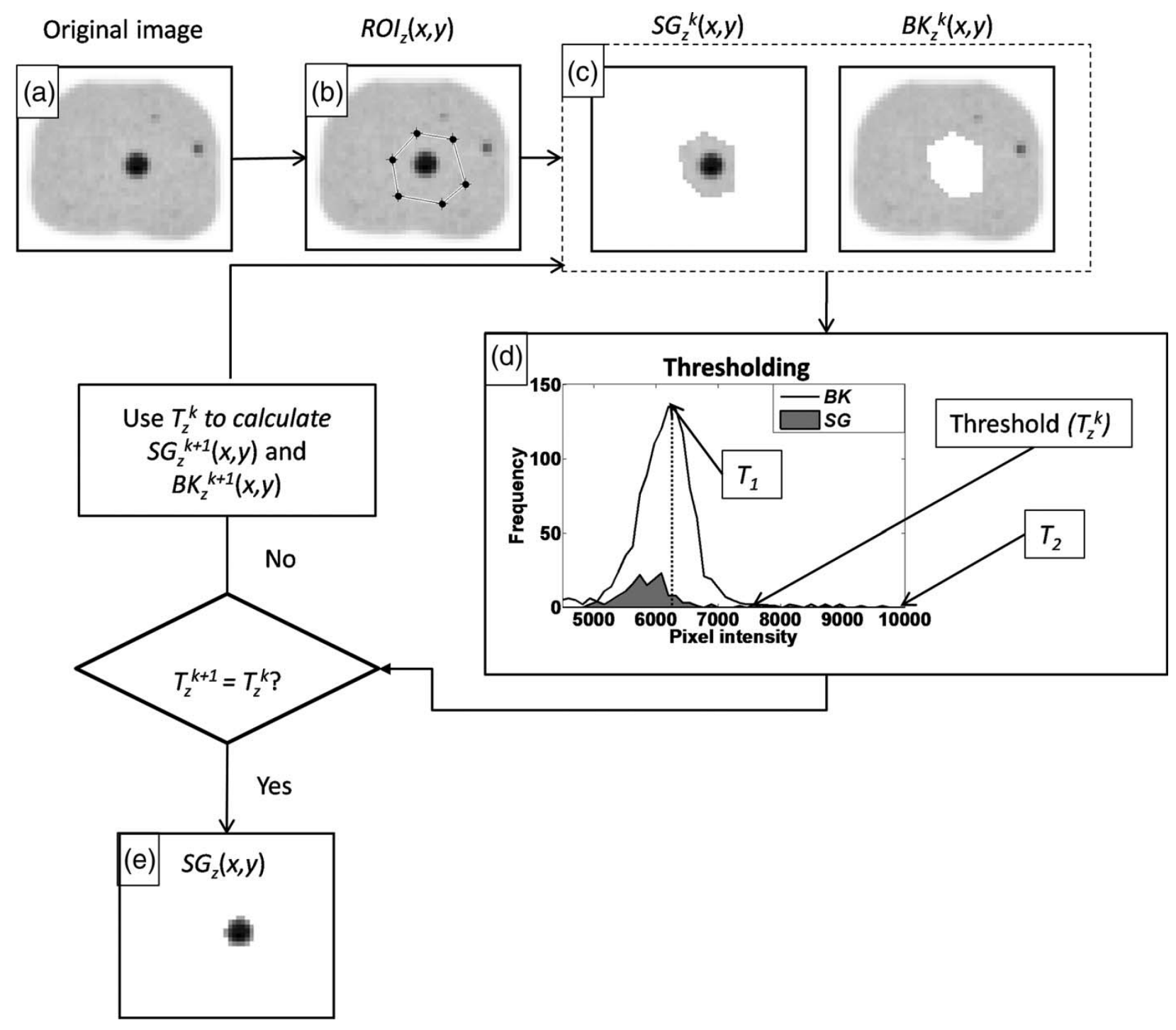

Figure 3.2 Block diagram of the HT algorithm with different components. (a) The original image on which the observer would draw a ROI. (b) Manually drawn ROI encompassing the selected tumor. (c) Initial segment $(S G)$ and background $(B K)$ obtained from the ROI. (d) Histogram of the background and the $S G$ are plotted on the same scale and $T_{z}^{k}$ is the threshold calculated between bins $T_{1}$ and $T_{2}$. (e) Once the stopping condition is satisfied the $S G$ derived includes the blur around the tumor. 
After attaining the subsequent binary mask by drawing the 3D ROI (called ' $R O I$ '), the initial value of $S G$ and $B K$ for $k=0$ is defined as $S G_{z}^{0}(x, y)=I_{z}(x, y) \bullet R O I_{z}(x, y)$ and $B K_{z}^{0}(x, y)=I_{z}(x, y)-S G_{z}^{0}(x, y)$, respectively (Figure 3.2.c), and ' $\bullet$ ' is the point wise multiplication.

The HT algorithm utilizes the histogram of $S G_{z}(x, y)$ and $B K_{z}(x, y)$ (Figure 3.2.d) to iteratively find the threshold value ( $\left.T_{z}^{k}\right)$ in the range between $T_{1}$ and $T_{2}$ (Equation 3.1). $T_{1}$ is the bin value, the value where $H_{B K}^{k}(T)$ is maximum and $T_{2}$ is the bin value of the last bin ' $P$ ' (Equation 3.1). The threshold is the point of intersection between the two histograms and Equations 3.1 and 3.2 shows how the value $T_{z}^{k}$ is calculated. As the algorithm is applied in $2 \mathrm{D}, T_{z}^{k}$ is calculated for each slice $(z)$.

$T_{z}^{k}=\left\{\begin{array}{lcc}\min \left(\left\{T: H_{B K}^{k}(T) \leq H_{S G}^{k}(T)\right\}\right) & \text { if } & \max \left(H_{B K}^{k}(T)\right) \geq \max \left(H_{S G}^{k}(T)\right) \\ \min \left(\left\{T: H_{B K}^{k}(T) \geq H_{S G}^{k}(T)\right\}\right) & \text { if } & \max \left(H_{B K}^{k}(T)\right) \leq \max \left(H_{S G}^{k}(T)\right) \\ 0 & \text { Otherwise }\end{array}\right.$

$T_{z}^{k} \in\left[T_{1} T_{2}\right] ;$ Where $T_{1}=\left\{T: \max \left\{H_{B K}^{k}(T)\right\}\right.$ and $T_{2}=T(P)$

The $T_{z}^{k}$ value achieved from Equations 3.1 and 3.2 is used to update $B K_{z}^{k}(x, y)$ and $S G_{z}^{k}(x, y)$ as described in Equations 3.3 and 3.4.

$$
\begin{aligned}
& B K_{z}^{k}(x, y)=I_{z}(x, y)-S G_{z}^{k}(x, y) \\
& S G_{z}^{k+1}(x, y)=\left\{\begin{array}{lll}
I_{z}(x, y) & \text { if } & S G_{z}^{k}(x, y) \geq T_{z}^{k} \\
0 & \text { Otherwise }
\end{array}\right.
\end{aligned}
$$


The algorithm is continued until the stopping condition $T_{z}^{k+1}=T_{z}^{k}$ is satisfied. Once the stopping condition is satisfied the segmented tumor $\left(S G^{k}\right)$ (Figure 3.2.e) achieved is not corrected for PVE and contains the blur pixels around the tumor.

In the case of multiple tumors in a homogeneous background the HT algorithm can be applied on all tumors simultaneously. Due to the inherent noisy nature of the PET image, the HT algorithm segments isolated small regions as tumors. These regions are just two dimensional noise regions and, based on their dimensionality, these noise regions are removed. The image derived after removal of the noise region is converted into a binary image $B I$ according to Equation 3.5 (below).

$$
B I(x, y, z)=\left\{\begin{array}{lll}
1 & \text { if } & S G(x, y, z)>0 \\
0 & & \text { Otherwise }
\end{array}\right.
$$

\subsection{THE IDTS ALGORITHM}

The $S G$ and $B I$ from the HT algorithm are now inputted to the second stage of the algorithm (in Figure 3.1 box called IDTS algorithm). The IDTS algorithm (after satisfaction of the stopping condition) outputs $T U$, where $T U$ is the binary mask of the PVE corrected segmented tumor. $T U$ is further used to calculate FV. The IDTS algorithm is a combination of two parts, deconvolution and the MHT algorithm. The IDTS algorithm is applied on each tumor individually. Henceforth the explanation of the algorithm is for finding the FV for a single tumor. The IDTS algorithm is the prime algorithm which corrects for the PVE, and the value of the $T U$ is dependent on the stopping conditions. The purpose of the stopping conditions is to automatically terminate the algorithm making it independent from arbitrarily choosing the number of iterations. A detailed explanation of the stopping conditions is given in section 3.4. 


\subsubsection{DECONVOLUTION METHOD}

PET images are always blurred by the camera's PSF, and deconvolution is often used to deblur the image. For accurate recovery of a non-blurred image, it is necessary to accurately estimate the PSF of the camera, which is quite challenging if the PSF is non-isotropic. The proposed algorithm uses the Van-Cittert's deconvolution method (Equation 3.6 below). In Equation 3.6, $\alpha$ is the convergence parameter which is set to value 1 and ' $\otimes$ ' is the convolution operator.

$D E_{z}^{k+1}(x, y)=D E_{z}^{k}(x, y)+\alpha\left(I_{z}(x, y)-P S F \otimes D E_{z}^{k}(x, y)\right)$

Where, $D E_{z}^{k+1}(x, y) \geq 0$ and $D E_{z}^{0}(x, y)=I_{z}(x, y)$

$P S F$ is the 2D Gaussian point spread function and $D E_{z}^{k}(x, y)$ is the deconvolved image. $D S_{z}^{k}(x, y)$ is the segment of a deconvolved image (given in Equation 3.7 below), which is used in MHT algorithm.

$D S_{z}^{k}(x, y)=D E_{z}^{k+1}(x, y) \bullet B I_{z}(x, y)$

To obtain $T U_{z}^{k}(x, y)$, the deconvolution algorithm is used at the onset to aid the MHT algorithm in segmentation of the blur around the tumor. The number of iterations $(k)$ required to obtain $T U_{z}^{k}(x, y)$ is dependent on the amount of blur present and the choice of PSF used in Equation 3.6.

\subsubsection{MODIFIED HISTOGRAM THRESHOLDING (MHT) ALGORITHM}

The MHT algorithm used is similar to the HT algorithm, where instead of $S G_{z}^{k}(x, y)$, $D S_{z}^{k}(x, y)$ is used to generate $H_{S G}^{k}(T)$. Unlike the HT algorithm, the value of $T(P)$ keeps 
updating with every iteration ' $k$ '. The value of each bin is $T(Q)=\left(\max \left(D S^{k} / P\right)\right) \times Q$. The $B K_{z}(x, y)$ is kept constant when applying the MHT algorithm (Equation 3.8). The histogram derived from $D S_{z}^{k}(x, y)$ and $B K_{z}(x, y), \quad H_{S G}^{k}(T)$ and $H_{B K}(T)$, respectively, is inputted in Equations 3.2 and 3.3 to obtain the threshold $\left(T_{z}^{k}\right)$.

$B K_{z}(x, y)=I_{z}(x, y)-S G_{z}(x, y)$

The background is not updated since now we are segmenting blur from the $S G_{z}^{k}(x, y)$, and the intensity of the blur is found to be mostly greater than the intensity of the background. When the tumor has heterogeneous activity distribution there is a possibility that the intensity of the blur on the edges could be greater than the intensity of some pixels within the tumor. So making the blur pixels part of the background can lead to segmentation of those pixels within the tumor.

The $D S_{z}^{k}(x, y)$ and $T_{z}^{k}$ are used to derive a binary image $T U_{z}^{k}(x, y)$, as given in Equation 3.9. $T U_{z}^{k}(x, y)$ includes the tumor whose blur is segmented.

$T U_{z}^{k}(x, y)= \begin{cases}1 & \text { if } \quad D S_{z}^{k}(x, y) \geq T_{z}^{k} \\ 0 & \text { Otherwise }\end{cases}$

$V O_{z}^{k}$ is the total number of voxels calculated from $T U_{z}^{k}(x, y)$ (Equation 3.10), and the product of total $\mathrm{VO}_{z}$ and volume of each voxel provides the $\mathrm{FV}$ (Equation 3.11) of a tumor, $V O_{z}^{k}=\sum_{x, y=1}^{X, Y} T U_{z}^{k}(x, y)$

$\mathrm{FV}=\sum_{z=1}^{Z} V O_{z} \times$ Volume of a voxel. 


\subsection{STOPPING CONDITION}

The main challenge in the development of this algorithm was in determining the most appropriate stopping condition which could lead to accurate volume estimations. As a result, two stopping conditions were deemed necessary: one that is applicable to tumors of all sizes having homogeneous activity uptake and another that is applicable when the size of the tumors is large $(>8 \mathrm{ml})$ and on tumors with heterogeneous activity uptake. The IDTS algorithm does not determine the applicability of the specific stopping condition depending on the nature of the tumor, and the algorithm is terminated when either one of the two stopping conditions is satisfied.

\subsubsection{FIRST STOPPING CONDITION}

In an ideal image which is not affected by blur and noise, the activity concentration (AC) is close to the maximum intensity for an object. For large tumors in PET images, the maximum intensity is close to the actual AC.

The value of maximum intensity ( $M V$ ) of $S G$ (Equation 3.12 below) reduces as the size of the tumor decreases due to PVE. The $M V$ of a tumor is still the value that is least affected by PVE.

$M V=\max (S G(x, y, z))$

Due to the application of the whole algorithm in 2D the algorithm calculates AC (Equation 3.13 below) of tumor on each slice (z) and compares it with $M V$. The first stopping condition is induced when $\mathrm{AC}$ is greater than or equal to $M V$ (Equation 3.14 below); this condition is checked for each iteration $(k)$ and each slice $(z)$. It is important to remember that the first stopping condition is applicable on tumors with homogeneous activity uptake, 
$\mathrm{AC}_{z}^{k}=\frac{\sum_{x, y, z=1}^{X, Y, Z} S G(x, y, z)}{V O_{z}^{k}}$

$\mathrm{AC}_{z}^{k} \geq M V$

\subsubsection{SECOND STOPPING CONDITION}

The second stopping condition is based on the nature of the deconvolution algorithm. It is most useful in segmenting tumors with heterogeneous activity uptake. The outcome of the deconvolution process is different for tumors whose volume is $<8 \mathrm{ml}$ in comparison to tumors whose volume is $\geq 8 \mathrm{ml}$. In Figure 3.3 (below) the value $D S_{z}^{k}(x, y)$ increases from the center and decreases towards the edges, whereas in Figure 3.3.b (below) the value of $D S_{z}^{k}(x, y)$ tends to zero at the center as well as towards the edges. It should be noted that the value of $D S_{z}^{k}(x, y)$ approaches zero towards the edges regardless of the size of the tumor. 

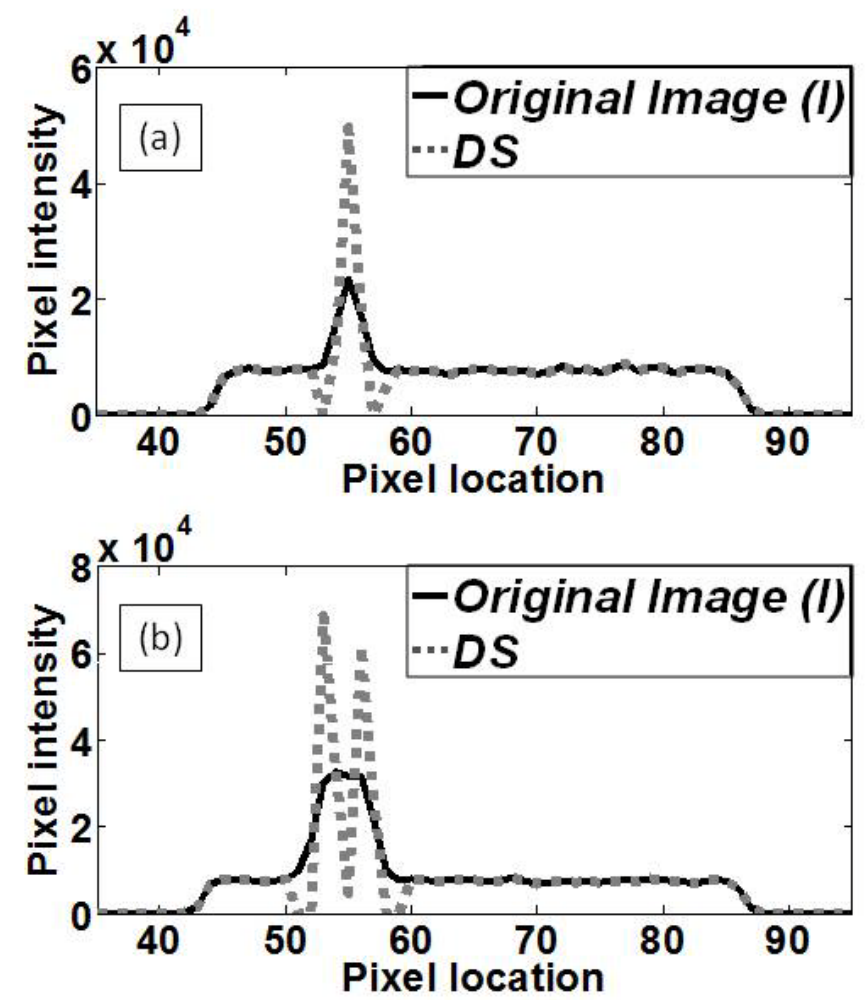

Figure 3.3 The deconvolution applied on the line signal (solid line) from a $4 \mathrm{ml}$ tumor (a) and 16 $\mathrm{ml}$ tumor (b) after 8 iterations. DS (dotted line) is the segmented deconvolved signal. (a) The value of $D S$ increases from the center and approaches zero at the edge. (b) The value of $D S$ approaches zero at the center and the edge.

This information should be considered to avoid segmentation of pixels from the central part of the tumor. The tendency of the value of $D S_{z}^{k}(x, y)$ to go to zero at the center for large and heterogeneous tumors is used to formulate the second stopping condition. The second stopping condition is actuated when a value of $D S_{z}^{k}(x, y)$ at the center goes below the threshold $\left(T_{z}^{k}\right)$ gained from the MHT algorithm, at which point the IDTS algorithm is terminated. For 
tumors with heterogeneous uptake, the second stopping condition is usually reached at a lower number of iterations compared to the first stopping condition. This property helps the algorithm from over or under estimating the FV. For the identification of the pixels belonging to the center region of $D S_{z}^{k}(x, y)$, a morphological operation of erosion is used.

\subsection{MAXIMUM INTENSITY CORRECTION}

At this stage of the process, the IDTS algorithm will return an overestimated FV for small tumors but a more accurately estimated FV for large tumors. The overestimation of FV hampers the performance of $\mathrm{AC}$ estimation as it is a function of FV (Equations 3.11 and 3.13). In order to get FV closer to the actual volume, it is necessary to compensate for the reduction of maximum intensity due to PVE, especially in small tumors $(<1.8 \mathrm{ml})$. Hence, it is necessary to develop a method by which a correction for the maximum intensity value $(M V)$ is possible. The main idea of MIC is to iteratively segment the blur pixels from $S G$ and add the mean value of the blur pixels $(M B V)$ to the $M V$. The algorithm is iteratively progressed until it arrives at the updated maximum value $(u M V)$. In Figure 3.4 (below), the MIC algorithm is implemented outside the original IDTS algorithm hence we use a different variable for the iteration number ' $j$ '. The variable with index ' $j$ ' would be identified as a variable derived from the MIC algorithm and value of $j=\{0,1,2 \ldots j\}$. 


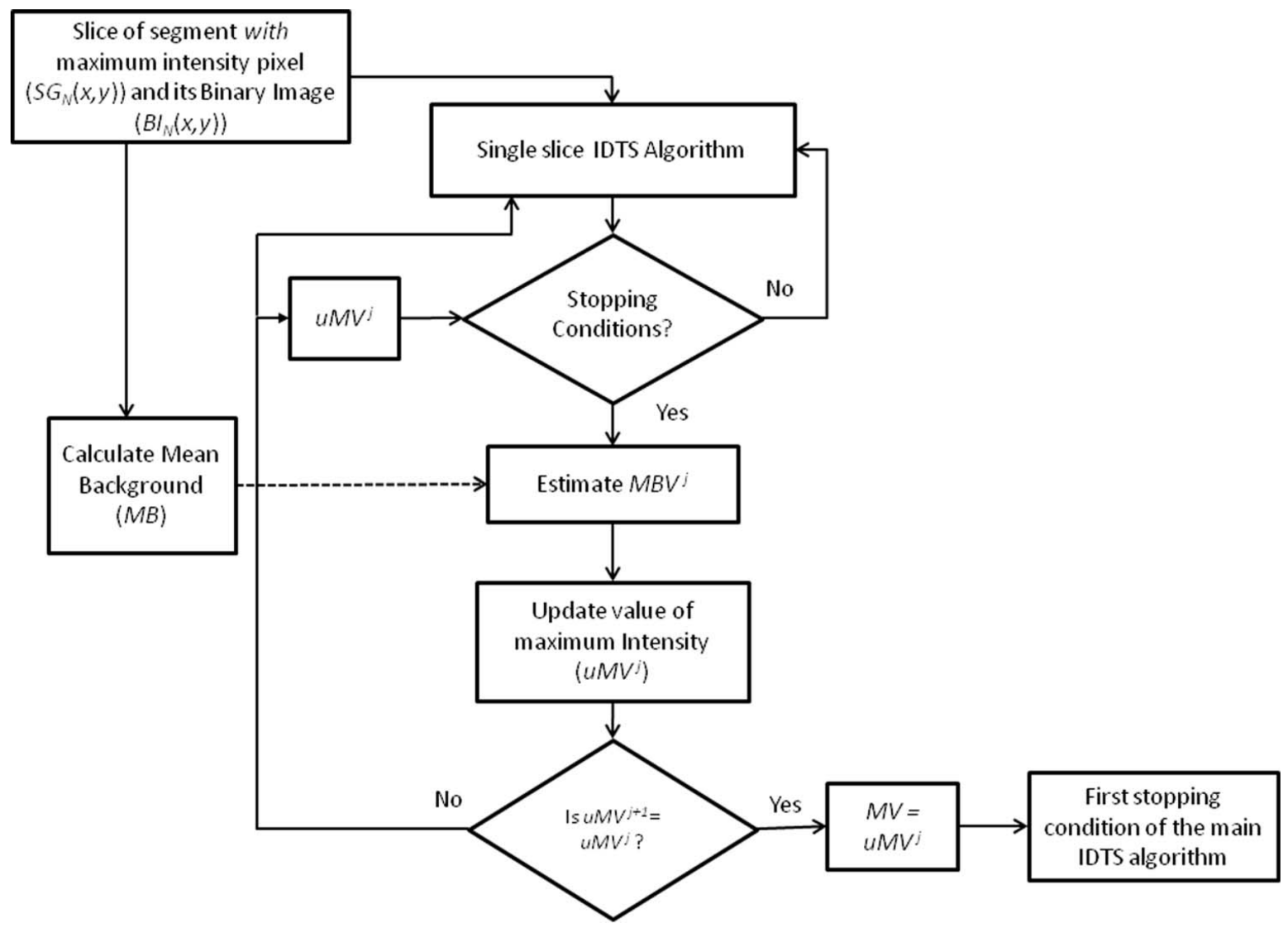

Figure 3.4 Complete block diagram of maximum intensity correction (MIC) algorithm. The output from the MIC is used as the stopping condition in the main IDTS algorithm.

After calculation of $u M V^{j}$, the IDTS algorithm will be re-applied and FV would be recalculated for a tumor. During the re-application of the IDTS algorithm the $u M V^{j}$ would be used in the first stopping condition instead of $M V$ (Equation 3.14). The MIC is only applied once on a tumor and an outline of the algorithm is shown in Figure 3.1.

It is observed from experiments that the greatest overestimation of FV occurred for $\mathrm{FV}<1.8 \mathrm{ml}$. Therefore, $1.8 \mathrm{ml}$ was chosen as a threshold to determine which tumor should be subjected to the MIC algorithm. The MIC algorithm is a combination of IDTS algorithm and 
stopping condition applied on one slice of $S G$, where ' $N$ ' is the slice number. The goal of the MIC algorithm is to calculate $u M V^{j}$, so the slice which contains the $M V$ pixel (the number of that slice is ' $N$ ') is selected to be subjected to the MIC algorithm. We choose one slice in the MIC algorithm because applying MIC in the whole 3D tumor leads to an overestimation of maximum intensity, which in turn leads to an underestimation of FV.

After identification of the qualified tumors for the MIC algorithm, the parameters $S G$ and the binary image $B I$ gained from HT algorithm are inputted into MIC algorithm. Once the slice number $N$ is identified from $S G, S G_{N}(x, y)$ and $B I_{N}(x, y)$ are subjected to the IDTS algorithm (within the MIC algorithm) until the stopping conditions are satisfied. Next $T U_{N}^{j}(x, y)$ and $V O_{N}^{j}$ are derived from the above step and used to identify the spill-out or blur pixels which in turn would be used to measure mean value of blur pixels $(M B V)$. From Equation 3.15 below, the $B R^{j}(x, y)$ is the binary $2 \mathrm{D}$ image displaying the location of the blur pixels.

$B R^{j}(x, y)=B I_{N}(x, y)-T U_{N}^{j}(x, y)$

The $B R^{j}(x, y)$ would be used to measure the $M B V^{j}$; however, for small tumors the spill-in is significant and must be accounted for. This is achieved by measuring the mean background $(M B)$ through application of dilation operation around $B I_{N}(x, y)$ as expressed in the following equation.

$D L(x, y)=\left(B I_{N}(x, y) \oplus S T(x, y)\right)-B I_{N}(x, y)$

$D L(x, y)$ contains all of the pixels that are outside of $B I_{N}(x, y)$ after application of the morphological operation of dilation (Equation 3.16), designated by the symbol ' $\oplus$ '. $S T(x, y)$ is the structuring element, which in this case is a unitary matrix of size $3 \times 3$. The dilation operation 
is used to estimate $M B$ because the pixels around the blur are most responsible for spill-in. The dilation for such a small tumor is chosen to be safe as there is less chance of including the intensity of neighboring tumors in the calculation of $M B$. In Figure 3.4, it is shown that $M B$ is calculated only once at the start.

$D L(x, y)$ measured from Equation 3.16 and $I_{N}(x, y)$ (where, $I_{N}(x, y)$ is a $2 \mathrm{D}$ image of the original input data $(I(x, y, z)))$ is used in the measurement of $M B$ (Equation 3.17).

$M B=\frac{\sum_{x, y=1}^{X, Y}\left(D L(x, y) \bullet I_{N}(x, y)\right)}{\sum_{x, y=1}^{X, Y} D L(x, y)}$

Furthermore, $M B$ would be used to measure $M B V^{j}$ (Equation 3.18 below). $M B$ is used in $M B V^{j}$ measurement because with small sized tumors with low SBR $(<5)$ the effect of spill-in is strong and the intensity of blur pixels is highly influenced by the intensity of the background. If the SBR is high, Equation 3.19 (given below) can be used instead to yield better results. Identifying the true SBR in any clinical image presents a challenge, thus the algorithm becomes impractical if it is dependent on the SBR. Hence, Equation 3.18 is used in the proposed algorithm in order to make this algorithm applicable under any condition.

$$
\begin{aligned}
M B V^{j} & =\frac{\sum_{x, y=1}^{X, Y}\left(B R^{j}(x, y) \bullet S G_{N}(x, y)\right)-\left(M B \times \sum_{x, y=1}^{X, Y} B R^{j}(x, y)\right)}{V O_{N}^{j}} \\
M B V^{j} & =\frac{\sum_{x, y=1}^{X, Y}\left(B R^{j}(x, y) \bullet S G_{N}(x, y)\right)}{V O_{N}^{j}}
\end{aligned}
$$


The $M B V^{j}$ gained from Equation 3.18 or Equation 3.19 is used to calculate $u M V^{j}$ (Equation 3.20) and the $u M V^{j}$ is be inputted in the stopping condition as shown in Figure 3.4. The algorithm continues till the condition $u M V^{j+1}=u M V^{j}$ is satisfied, then the $u M V$ is injected in the stopping condition of the main algorithm (Figures 3.1 and 3. 4).

$u M V^{j}=M V+M B V^{j}$ 


\subsection{ALTERED HISTOGRAM THRESHOLDING (FOR CLINICAL IMAGES)}

The HT algorithm, when applied on some clinical images, fails to find the correct threshold and thereby fails to segment the tumor. The clinical images, unlike phantom images, have high heterogeneous activity profile in the background; this causes the histogram to be noisy. The noisy histogram is the main reason why the HT algorithm fails The noisy histogram affects the HT algorithmbecause it uses Equations 3.1 and 3.2 to find the threshold, where the threshold is the point of intersection between the histogram of the background $\left(H_{B K}^{k}(T)\right)$ and the histogram of the segment $\left(H_{S G}^{k}(T)\right)$, where $T$ is the bin value. With threshold $\left(T^{k}\right)$ at every iteration $\mathrm{k}$, the algorithm updates the background image $(B K)$ and segment image $(S G)$, given in Equations 3.3 and 3.4 .

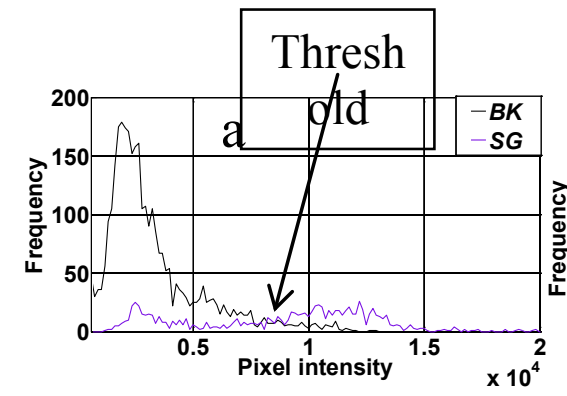

Iteration 1

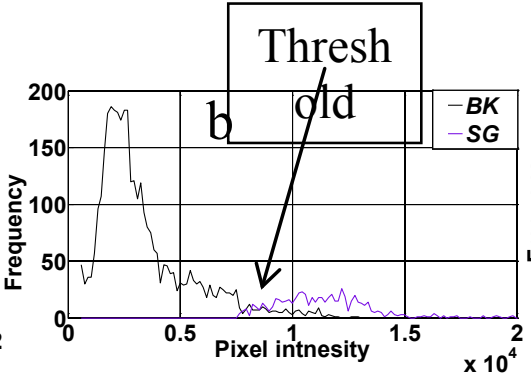

Iterations

5

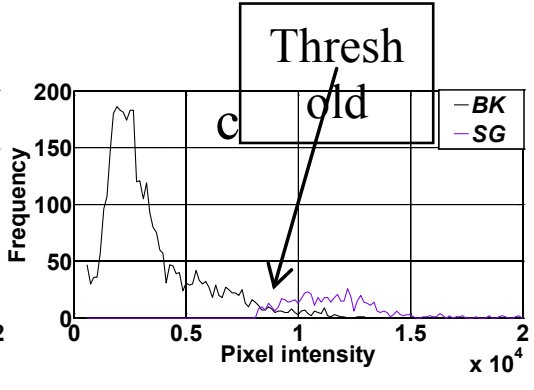

Iterations

30

Figure 3.5 The progression of HT algorithm after (a) iteration 1, (b) iteration 5 and (c) iteration 30.

Figure 3.5 shows histograms of $B K$ and $S G$ after the application of HT algorithm at iterations 1,5 and 30 . The threshold fails to moves forward after 5 iterations and its value remains the same after 30 iterations. In Figure 3.5 we see that there are many bins still overlapping on each other. This is the feature which shows that the HT algorithm has failed. The 
overlapping features of the histograms were similar for all clinical cases on which the algorithm was applied and failed. Hence, the overlapping feature was used to solve the failing of the HT algorithm in the clinical image by altering the method for calculating the threshold. In the original HT algorithm, the algorithm was stopped when the threshold $\left(T^{k}\right)$ equals $T^{k-1}$ with $T^{k}$ the final threshold after $k$ iterations. From section 3.2 we know that $T$ is the bin value, $Q$ is the bin number $\{0,1,2, . ., P\}$ and $P$ is the total number of bins. We also showed how bin value $(T)$ is the function of bin number $(Q)$ and how its calculation is done using the equation $T(Q)=(\max (I(x, y, z)) / P) \times Q$.

$T_{z}^{k}=\max (I(x, y, z) / P) \times Q_{z}^{k}+1 \quad$ if $\quad H_{S G}^{k}(T)>0$

We observed that if the threshold was moved by one bin the algorithm was able to segment the tumor. After identifying if the HT algorithm has failed we moved the threshold by one bin thereby altering the HT algorithm. The formulation of new threshold in the altered HT algorithm is given in Equation 3.21, which is applied after two conditions are satisfied, one is that stopping condition $T_{z}^{k}=T_{z}^{k-1}$ is reached and other is the condition when the value of the histogram of the segment is greater than zero at the threshold $T_{z}^{k}$ and it is given by $H_{S G}^{k}\left(T_{z}^{k}\right)>$ 0.

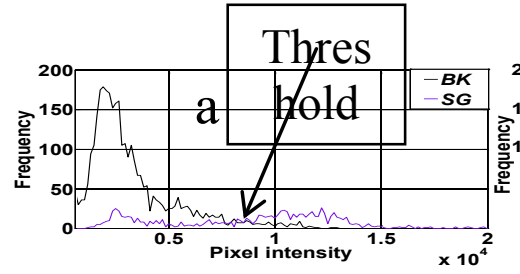

Iteration

1

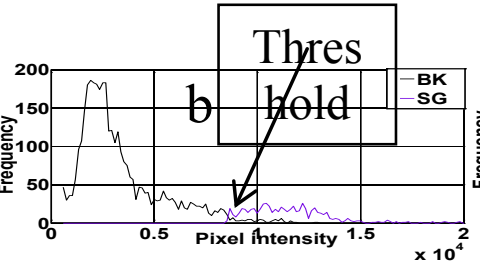

Iterations

5

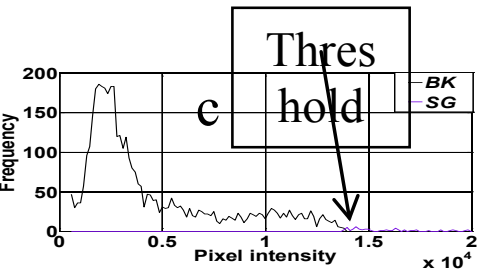

Iterations

30 
Figure 3.6 The progression of altered HT algorithm with at (a) iteration 1, (b) iteration 5 and (c) iteration 30 .
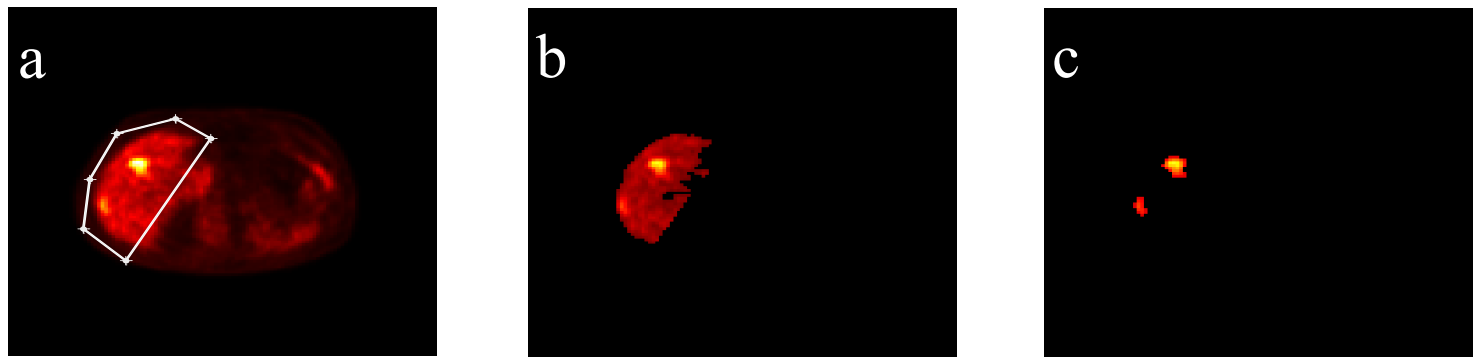

Figure 3.7 (a) Original image with ROI. (b) The segmentation of the tumor fails with the application of the original HT algorithm. (c) The altered HT algorithm was able to successfully segment the tumors.

From Figure 3.6 we can see that the histogram of BK and SG get well separated by the time the altered HT algorithm reaches 30 iterations. Figure 3.7 (a) shows the original image on which both the HT and altered HT algorithm was applied and its results are shown in Figure 3.7 (b) and Figure 3.7 (c), respectively. The altered HT algorithm was successful in separating background and tumor, whereas the original HT algorithm completely failed.

\subsection{DISCUSSION}

During the formulation of the HT algorithm the fact that data in the clinical images would have more heterogeneous activity profile compared to the phantom data was considered. That is the reason why the algorithm was applied in $2 \mathrm{D}$ instead of $3 \mathrm{D}$. The effectiveness of the 2D compared to $3 \mathrm{D}$ was never tested in phantom data because it is very difficult to create heterogeneous activity profile in the physical phantom, but it is possible to mimic the condition in the simulated phantom. The altered HT algorithm is applied only once the stopping condition is satisfied; this pre-condition increases the computational time, but not by much. 


\subsection{CONCLUSION}

The altered HT algorithm performance can be further tested on simulated phantom data with heterogeneous activity profile in the background. If the altered HT algorithm works on clinical data it should also work in the phantom data. The new values FV and $\mathrm{mAC}$ in phantom data is calculated by application of the altered HT algorithm and the new results are compared with results acquired from the original HT algorithm. The altered HT will change the FV and mAC values but it should not be significant. 


\section{CHAPTER 4}

Volume of interest generating algorithm

\subsection{INTRODUCTION}

The iterative deconvolution thresholding segmentation (IDTS) algorithm is semiautomatic because it requires the user to draw a region of interest (ROI) at the first step. The user has to decide which tumor is to be segmented or the user can segment multiple tumors within a specific organ. In phantom data the ROI was drawn on one transverse slice, which the user had to select, and that one ROI was used for all the slices. This approach is impractical in clinical images where many organs have activity profiles similar to the tumors. The algorithm should also be capable of segmenting tumors from a specific organ as there are therapies like selective internal radiation therapy (SIRT) which would only require volume of tumors in liver. As the efficicy of a therapy is dependent on segmenting tumors from the right organ the single slice ROI drawing is modified into a volume of interest (VOI) generating algorithm. The VOI generating algorithm makes the application of the IDTS algorithm in the clinical data less time consuming. The VOI still requires the user to draw the ROI but instead of drawing on one transverse slice, three ROIs have to be drawn, one in the coronal slice followed by the sagittal slice and than last in the transverse slice.

\subsection{PROBLEM WITH DRAWING ONE REGION OF INTEREST}

There are organs like kidney, spine, heart, bladder and many others which can sometimes show activity similar to the tumor. An algorithm which attempts to automatically segment tumors without the information of the organ in which the tumor is located may result in 
segmenting any of the above mentioned organs unintentionally. Segmenting organs together with the tumor would render any algorithm useless. It will also reduce the confidence of the radiologist towards the results obtained. If the algorithm is not able to differentiate between tumor and organ, the process would be very time consuming as the radiologist would have reanalyze results and manully remove all the organs before applying the segmentation algorithm.

Figures 4.1-4.3 shows examples of clinical images where the activity of the kidney, heart, and spine, respectively are similar to the activity of the tumor. In Figure 4.1.a we observe that the kidney has a similar activity profile as the tumor and hence a ROI on that slice, avoiding the kidney, will not ensure that the same ROI will not include the kidney on another slice. To avoid this problem we might want to draw the ROI in the sagittal view as the kidney location is clearly visible. However, that choice cannot be universally applied to all images as can be seen in Figure 4.2, which shows that in the sagittal view we would fail to see the heart clearly. A similar problem is demonstrated in the coronal view as seen in Figure 4.3, where it is difficult to draw a ROI without avoiding the spine. In order to overcome this problem we have developed a VOI generating algorithm. 

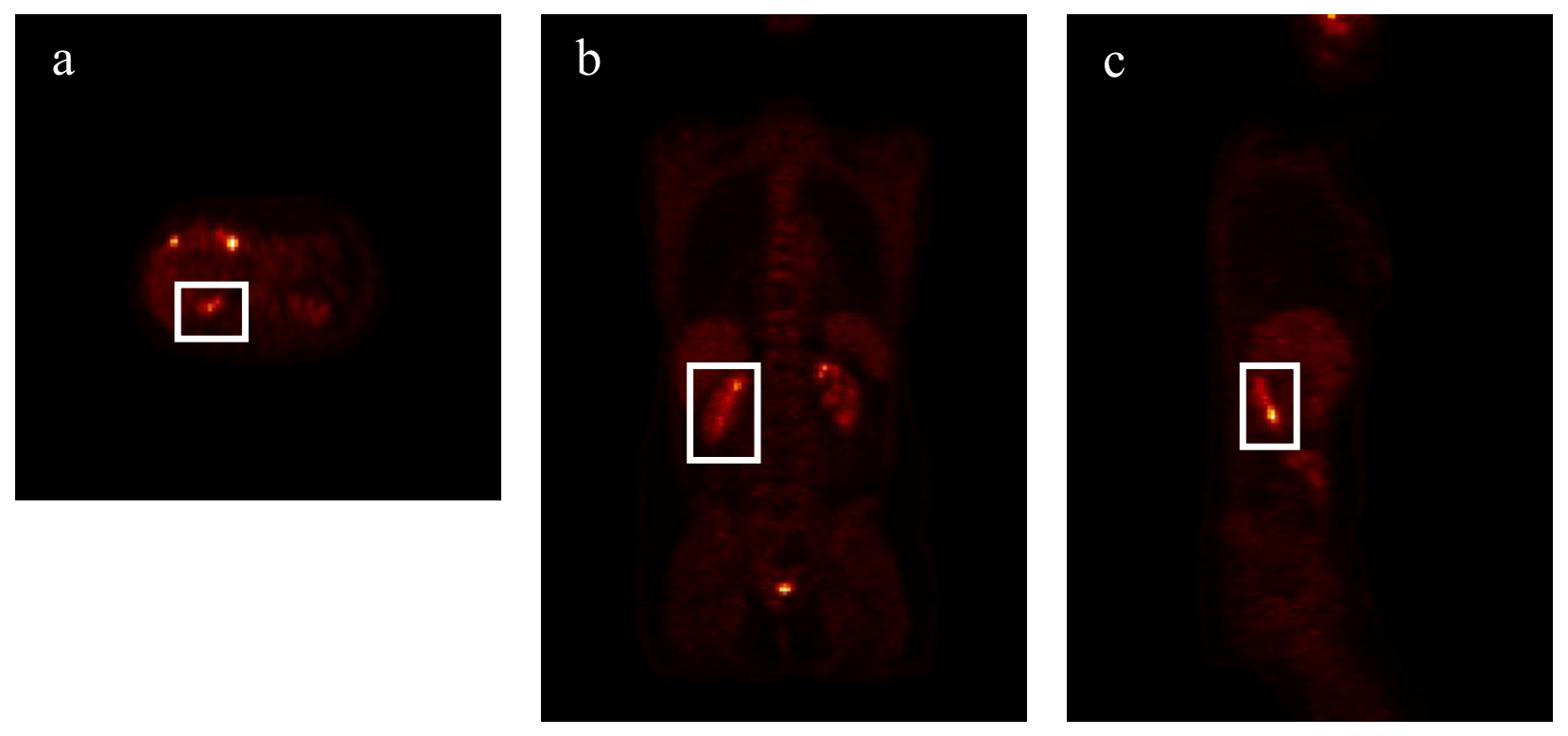

\section{Transverse Sagittal}

\section{Coronal}

Figure 4.1 Example of clincial data where the activity of the kidney is similar to the tumor (a) transverse slice (b) coronal slice (c) sagittal slice. 


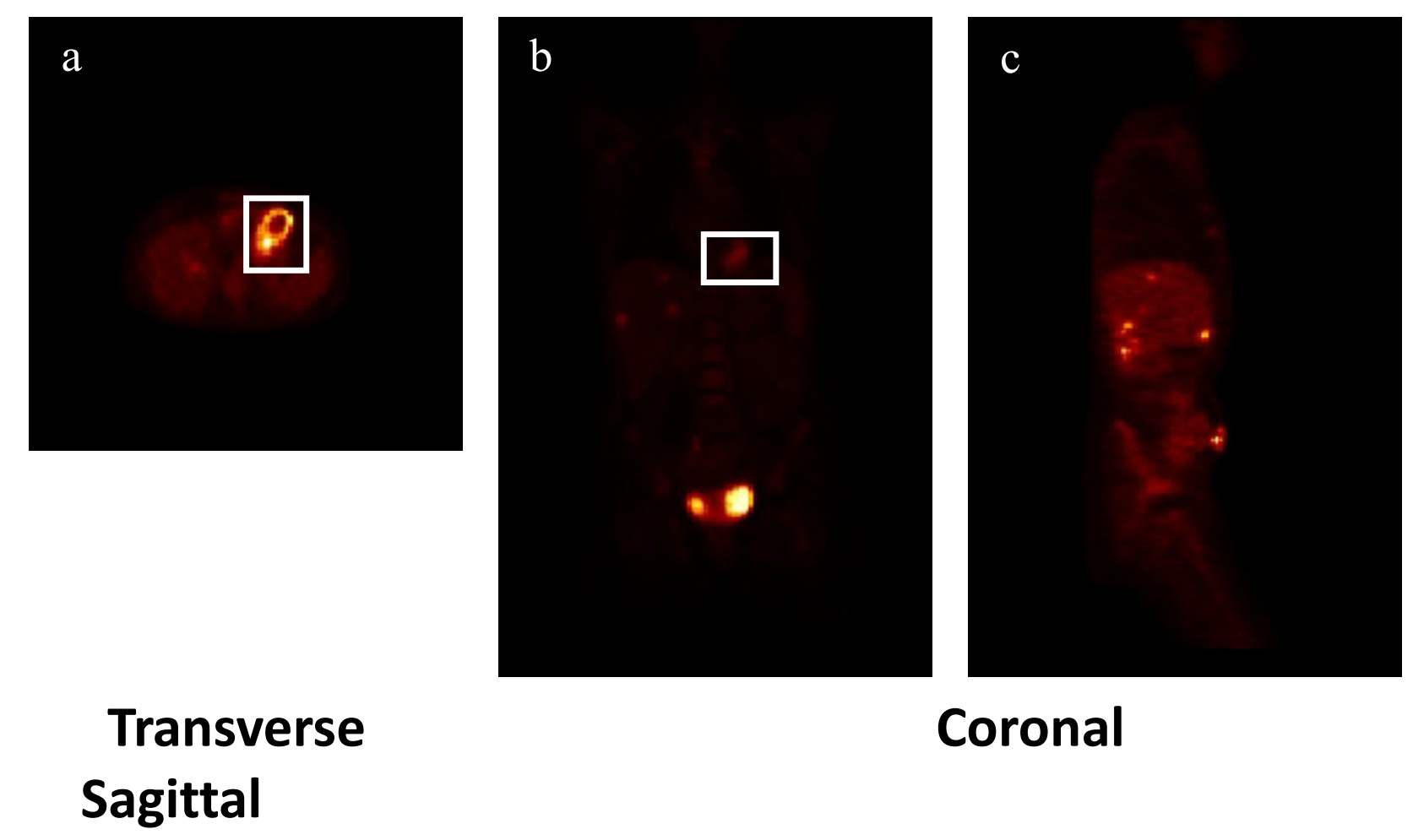

Figure 4.2 Example of clincial data where the activity of the heart is similar to the tumor (a) transverse slice (b) coronal slice (c) sagittal slice. 

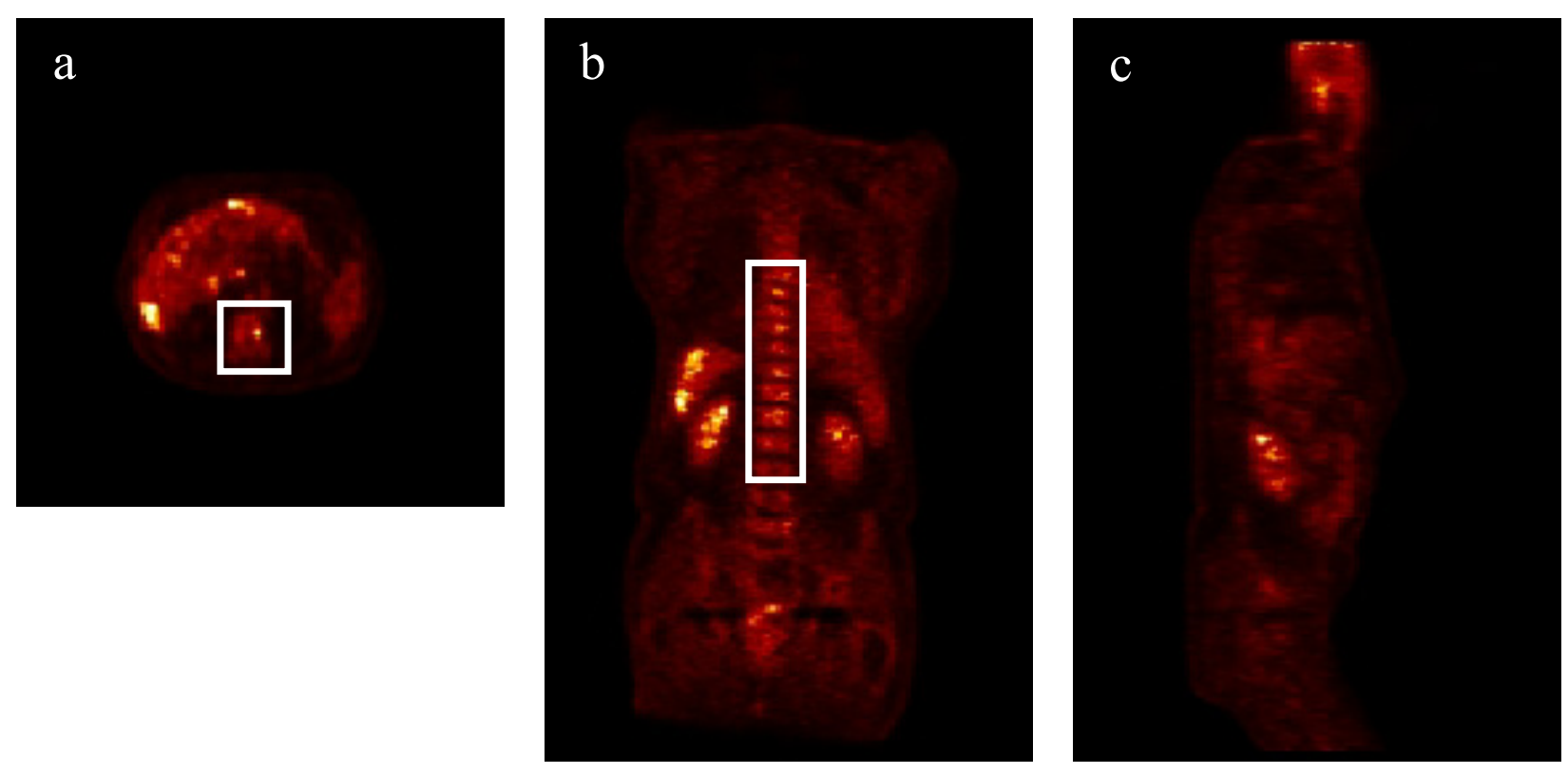

\section{Transverse Sagittal}

\section{Coronal}

Figure 4.3 Example of clincial data where the activity of the spine is similar to the tumor (a) transverse slice (b) coronal slice (c) sagittal slice.

\subsection{VOLUME OF INTEREST GENERATING ALGORITHM}

The VOI generating algorithm first genarates a maximum intensity projected (MIP) image in the coronal view of a 3D image $I(x, y, z)$ where $x=[1,2, . ., X], y=[1,2, . ., Y]$ and $z=[1,2, . ., Z]$. The MIP in the coronal view is the maximum of image $I(x, y, z)$ in the $y$ direction and is givien by $I_{\text {coronal }}(x, z)=\max _{y} I(x, y . z)$. Similarly MIP in sagittal and transverse are the maximum of image $I(x, y, z)$ in $x$ and $z$ direction and are given by $I_{\text {sagittal }}(y, z)=\max _{x} I(x, y \cdot z)$ and $I_{\text {transverse }}(x, y)=\max _{z} I(x, y . z)$, respectively. 


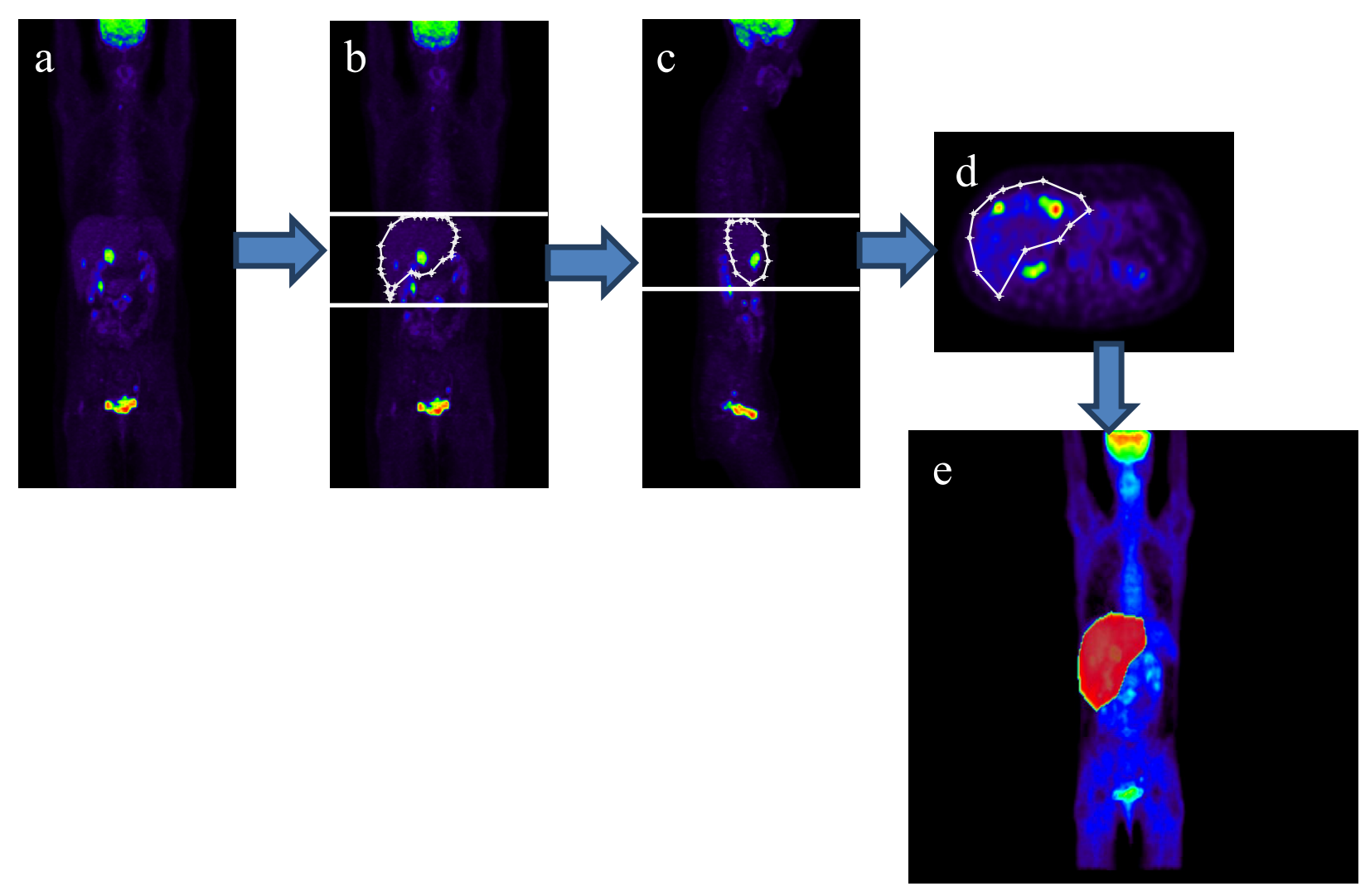

Figure 4.4 Outline of the VOI generating algorithm (a) Original image (b) ROI on MIP in coronal view (c) ROI on MIP in sagittal view (d) ROI on MIP image in transverse view and (e) showing 3D rendering of the VOI (red).

Figure 4.4 provides the outline of the overall algorithm. First a ROI is drawn in the coronal MIP image $I_{\text {coronal }}(x, z)$ and given by $R O I_{\text {coronal }}(x, z)$. It is followed by drawing ROI in sagittal MIP image given by $I_{\text {sagittal }}(x, z)$ called $R O I_{\text {sagittal }}(x, z)$ the formulation of transverse MIP is given below 
$I_{\text {transverse }}(x, y)=\max _{z}\left(I(x, y, z) \bullet R O I_{\text {coronal }}(x, y)\right)$

Equation 4.1 provides the coronal MIP in a transverse slice generated between the ROI in the coronal view. In Figure 4.4.b, the limits of the $R O I_{\text {coronal }}(x, z)$ in $z$ direction are used to genrate $\operatorname{ROI}_{\text {transverse }}(x, z)$. The intersection between three ROI is the VOI and is given in Equation 4.2 below.

$V O I=R O I_{\text {coronal }}(x, z) \cap R O I_{\text {sagittal }}(y, z) \cap R O I_{\text {transverse }}(x, y)$

Figures 4.5 and 4.6 show clinical data from two patients. In both cases, the VOI was able to successfully surround the region in the liver and avoid the kidney and other organs. 


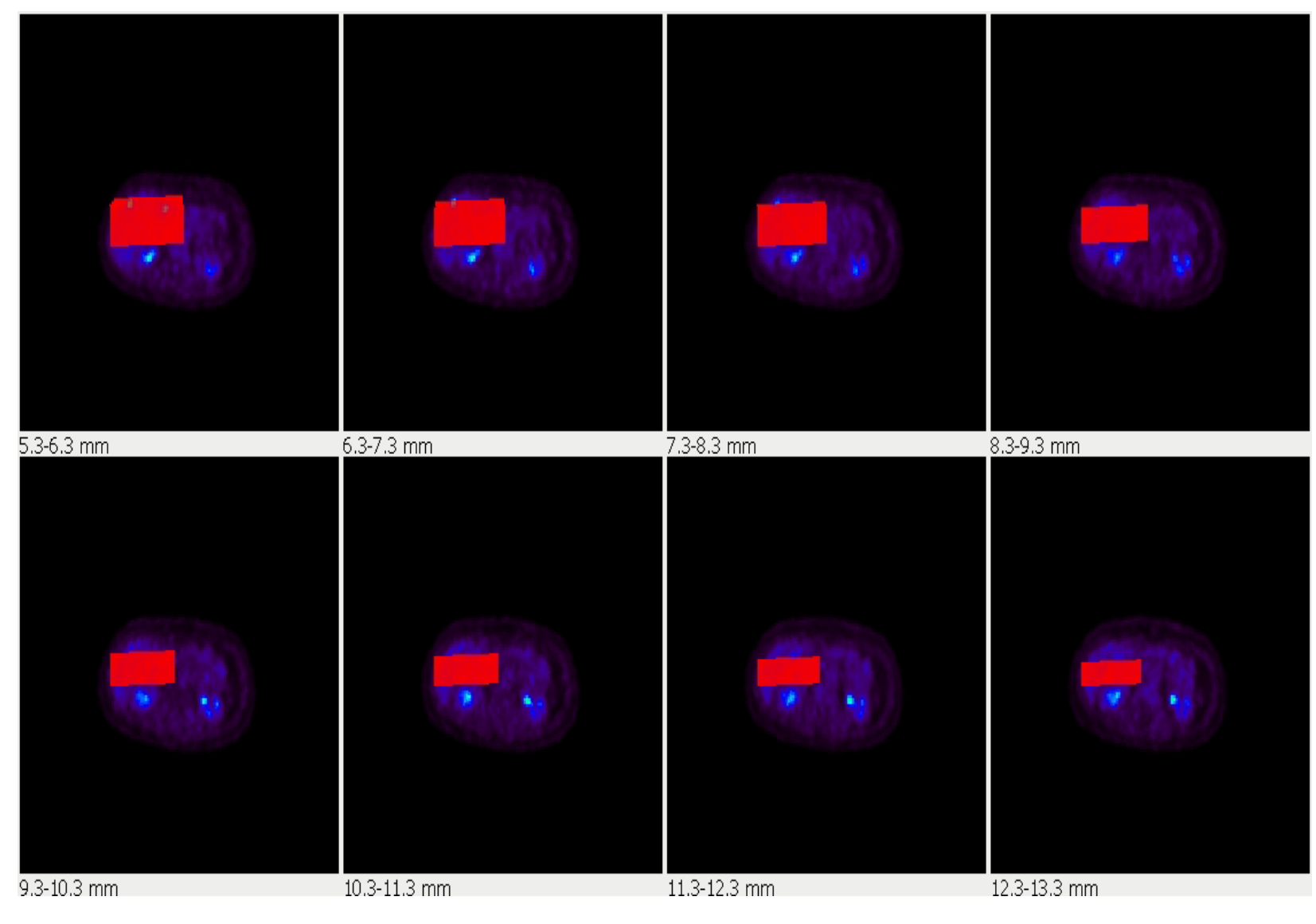

Figure 4.5 Transverse view of slices of patient 1 where the VOI (red) only surround the region in the liver and avoids the kidney and spine. 


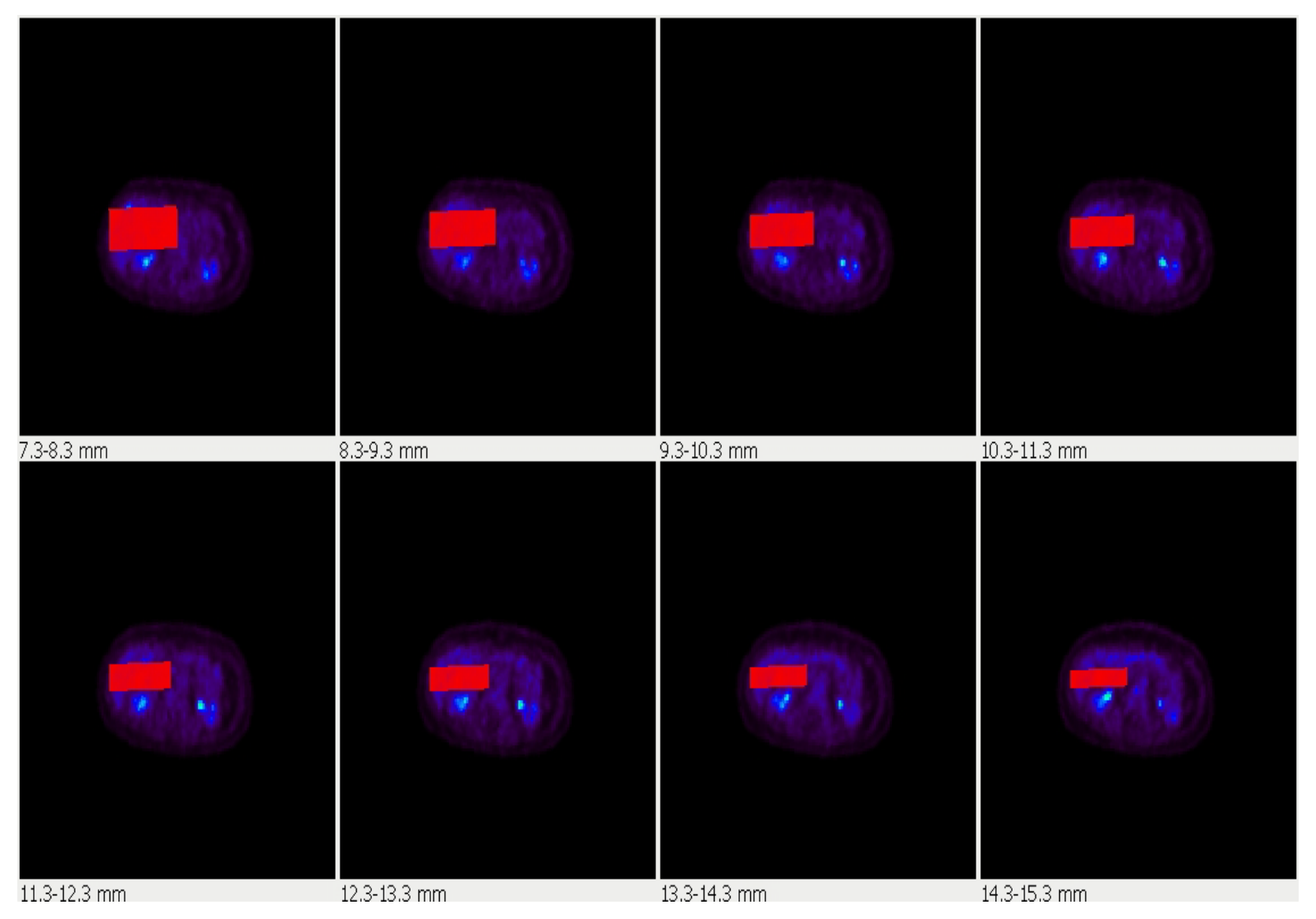

Figure 4.6 Transverse view of slices of patient 2 where the VOI (red) only surround the region in the liver and avoids the kidney and spine.

\subsection{DISCUSSION}

The method proposed herein has increased the user input from drawing the ROI on one slice to drawing the ROI on three different views. Li, et al and Dewalle-Vignion, et al have generated VOIs where in the case of Li, et al the VOI was just a 3D box and in the case of DewalleVignion, et al the VOI was generated using MIP images [31, 52]. But in Dewalle-Vignion et al the VOI was only applied to tumors in head and neck where the occurrences of multiple organs and tumors in the neighboring region are not visible. 


\subsection{CONCLUSION}

The proposed algorithm of VOI generation is simple and fast and would lend confidence to the results obtained. The usefulness of the algorithm still needs to be tested in the clinical environment where the input of the users and their learning curve have to be considered. Some knowledge about how optimally a person can draw VOI will need to be obtained. 


\title{
CHAPTER 5
}

\author{
Results
}

\subsection{PHYSICAL PHANTOM STUDIES (HOMOGENEOUS TUMORS)}

\subsubsection{INTRODUCTION}

The partial volume effect (PVE) causes the activity in the tumor to spill out into the background; the counts are preserved but spread over a larger area. Definitively validating tumor volume in clinical data presents a challenge, making it important to validate the performance of the algorithm first in the phantom data. The phantom data represents simpler cases compared to clinical data, but if an algorithm fails to perform on phantom data it is unlikely to work on clinical data. Validation of clinical data is presented in later section.

The phantom data are categorized into two types: physical and simulated. Physical phantom data is generated by taking images of a plastic phantom that is filled with water and radio-isotope using a PET camera. Most of the manufacturers of physical phantoms provide spherical inserts which also can be filled with water and radio-isotope in order to simulate a tumor. The anthropomorphic mathematical models like NCAT and ZUBAL are used to generate simulated phantoms $[53,54]$. The simulated phantoms are further sub-divided into simple and complex. A simple simulated phantom is generated by convolving Tthe image with a point spread function (PSF) and adding Gaussian noise. The PSF is a Gaussian function whose full width half maximum (FWHM) is equal to the camera's spatial resolution [42]. The standard deviation of Gaussian noise is inversely proportional to the scan time. A complex simulated phantom data is generated by using the Monte-Carlo method to simulate the physical process and 
instrumentation of a PET camera. The software packages that can be used to generate MonteCarlo based projection data are GATE, SimSET and PET-SORETO [55-57]. Once the projection data is achieved they are reconstructed and an open source software that is widely used is called software for topographic imaging reconstruction (STIR) [58].

\subsubsection{PHYSICAL PHANTOM DATA}

In order to validate our algorithm, physical phantom studies were performed using the LiquiPhil Organ Scanning phantom manufactured by the Phantom Laboratory. The volume of the phantom is $11 \mathrm{~L}$. The tumors were represented by hollow spheres of different sizes $(0.5,1,2$, $4,8$ and $16 \mathrm{ml})$. They were filled with water and ${ }^{18} \mathrm{~F}-\mathrm{FDG}^{7}(1$ or $2 \mathrm{mCi} / \mathrm{L})$ and inserted into the phantom which was filled with water and ${ }^{18} \mathrm{~F}-\mathrm{FDG}(0.13-0.45 \mathrm{mCi} / \mathrm{L})$ for different SBRs $(2.75$, 4.4, 5.5, 7.3 and 8.8).

The imaging experiments were performed using a GE Discovery Light Speed scanner at the Baptist Hospital of Miami. The size of the entire image was 128 x 128 × 83 or 192 x $192 \times 83$, and the dimensions of each voxel were $5.46 \times 5.46 \times 3.27 \mathrm{~mm}$ or $3.64 \times 3.64 \times 3.27 \mathrm{~mm}$ respectively. The acquisition was performed at two bed positions using list-mode and the time for each scan was five minutes. To compare the effects of count statistics, scans for three-minute and one minute durations were derived from the five-minute list-mode data. The image reconstructions were performed using the camera manufacturer's built-in VUE point algorithm with two iterations and 18 subsets; the cut-off filter was $6.4 \mathrm{~mm}$. The VUE point algorithm is a maximum likelihood ordered subset expectation maximization (ML-OS-EM) iterative reconstruction algorithm which incorporates the random and scatter correction in the ML-OSEM equation [59].

\footnotetext{
${ }^{7}$ Floride-18-Fluro-deoxy-glucose
} 
The algorithm was tested by varying the parameters in the algorithm, as well as the SBR and image acquisition time, which affects the overall image counts. Within the algorithm, the parameters that were changed were the shape and size of the manual ROI, the FWHM of the Gaussian PSF used in the deconvolution algorithm, and the size of the Gaussian PSF.

\subsubsection{TESTING THE IMPACT OF MANUAL DRAWING OF REGION OF INTEREST}

The drawing of region of interest (ROI) is the only manual input required to start the algorithm. As this is the starting step, it is critical that the shape and size of the ROI does not impact the final result.

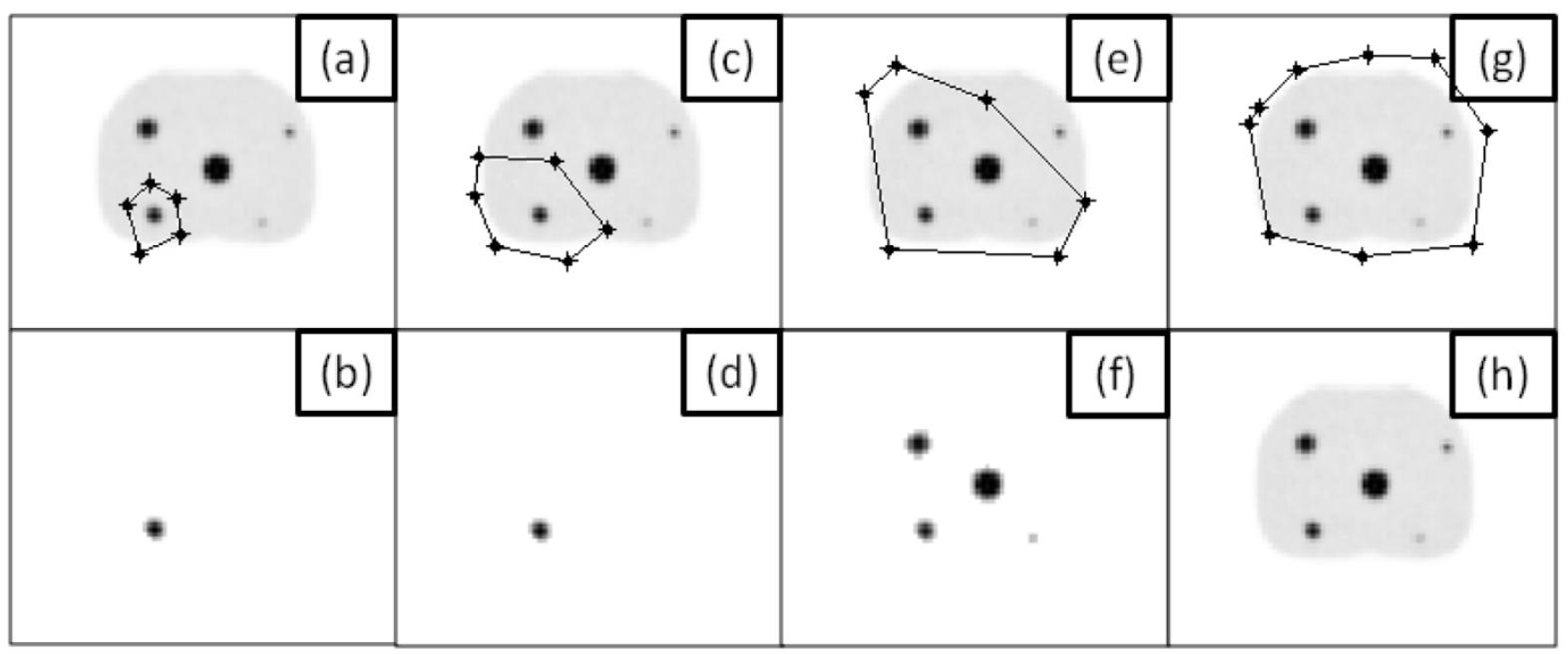

Figure 5.1 Three types of manually drawn ROI (a) The ROI is drawn on every slice close to the tumor. (c) The ROI is drawn on one slice and drawn bigger than the tumor. (e) The ROI drawn includes multiple tumors. (g) A very large ROI is drawn on one slice. (b, d, f and h) Result after segmentation, where in $\mathrm{h}$, the segmentation completely fails. 
To explore the limits of what shape and size of ROI we can draw, four methods of ROI drawing were tested (Figure 5.1). In the first method the ROI was drawn on every slice where the tumor was visible. As this method is considered the most accurate, it is compared with the other three methods (Figure 5.1. a). The second method involved drawing the ROI only on one slice and projecting that ROI onto every other slice (Figure 5.1. c). The third method involved drawing the ROI over multiple tumors in one slice (Figure 5.1. e). And the fourth method involved drawing a very large ROI with only a few pixels outside the ROI (Figure 5.1. g). The volume obtained from the second and third method of drawing the ROI was the same as with the first method. The segmented tumors in the first three methods were highly correlated spatially (Figures $5.1 \mathrm{~b}, \mathrm{~d}$ and f). But with the fourth method (Figure $5.1 \mathrm{~h}$ ) the algorithm completely failed to segment tumors from the background.

\subsubsection{RESULTS FROM HT ALGORITHM}

For the algorithm to successfully estimate FV it is necessary to segment the tumors with their entire associated blur from the background. Moreover, the SG volume (SG volume is the volume of segment $S G$ ) should always be greater than the true volume. The FV is highly dependent on the SG volume, and any change in the SG volume greatly affects the final FV. 


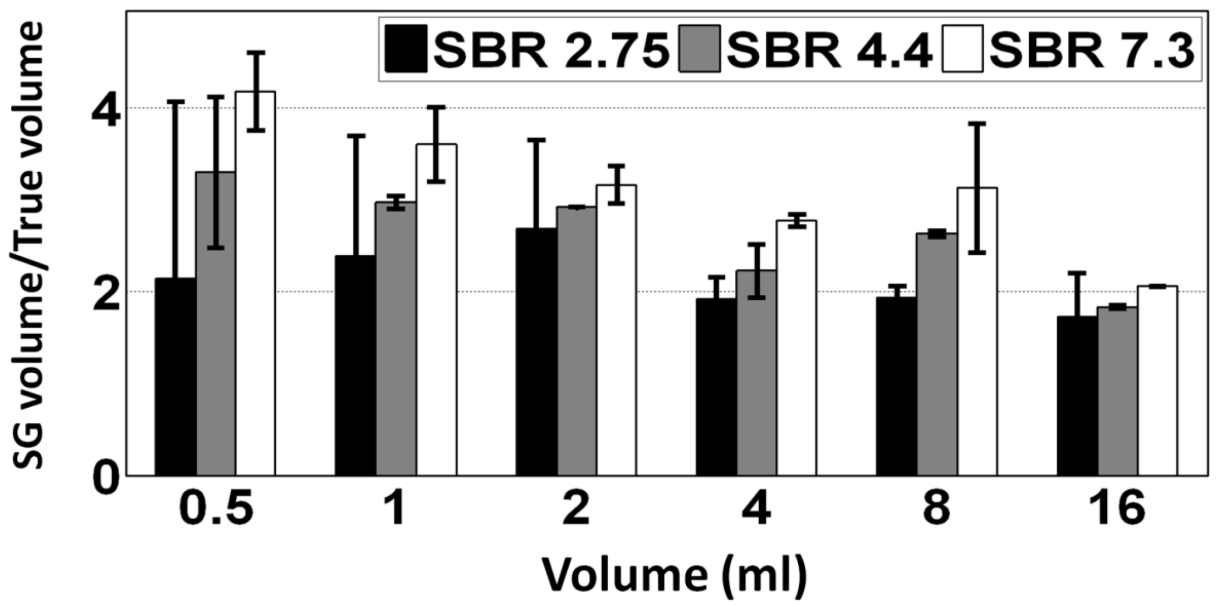

Figure 5.2 The ratio of SG volume/True volume after HT algorithm for different SBR.

Figure 5.2 shows a plot of the ratio of SG volume to true volume for different SBR and for different tumor sizes. The plot shows SG volume for a 5 minute scan time and pixel size of 5.46 $\mathrm{mm}$; SG volume for 1 and 3 minute scan times and $3.64 \mathrm{~mm}$ pixel sizes are not shown because they all show a similar pattern. SG volume is almost double the true volume for all sizes of tumors and the ratio of SG volume to true volume increases as the size of the tumor decreases. The amount of blur increases as the SBR increases and (SG volume)/(true volume) ratio shows that blur is dependent on SBR. The ratio of the SG volume to true volume is lowest at SBR of 2.75, and the ratio increases as the SBR increases (Figure 5.2). In Figure 5.2 it can be seen that there is a large variation in the ratio of SG volume to true volume in small tumors $(<2 \mathrm{ml})$ for SBR of 2.75 compared to SBR of 7.3. The increase in tumor size reduces the variation in the 
measurement of (SG volume)/(true volume). The variation is the standard deviation between two scans with the same SBR, acquisition time and reconstruction parameters.

\subsubsection{THE FINAL FV AND RC AND ROBUSTNESS OF THE IDTS ALGORITHM}

The SG volume was used as the input for the IDTS algorithm to calculate the final FV. This step was fully automated and the performance of the algorithm was tested by varying different parameters. The performance of the IDTS algorithm upon varying different parameters is demonstrated in Figures 5.3-5.8 for different pixel sizes of $5.46 \mathrm{~mm}$ and $3.64 \mathrm{~mm}$. The error bars in Figure 5.3-5.8 correspond to the standard deviation between two phantom data sets with same SBR and acquisition time. Figures 5.3, 5.5 and 5.7 are plots showing results of the FV estimation in terms of percentage of true volume ( $\%$ of true volume), which is given by Equation 5.1 below.

Figures 5.4, 5.6 and 5.8 are plots showing results of AC estimation and are plotted in terms of $\mathrm{RC}$, where $\mathrm{RC}$ is ration of measured $\mathrm{AC}$ by known $\mathrm{AC}$. (Equation 5.2 below).

$\%$ of true volume $=\frac{\mathrm{FV}}{\text { true volume }} \times 100$

$\mathrm{RC}=\frac{\mathrm{AC}}{\text { Known } \mathrm{AC}} \times 100$

Where FV is the measured functional volume of the segmented tumor (Equation 3.11) and the $\mathrm{AC}$ is measured using Equation 3.13. 


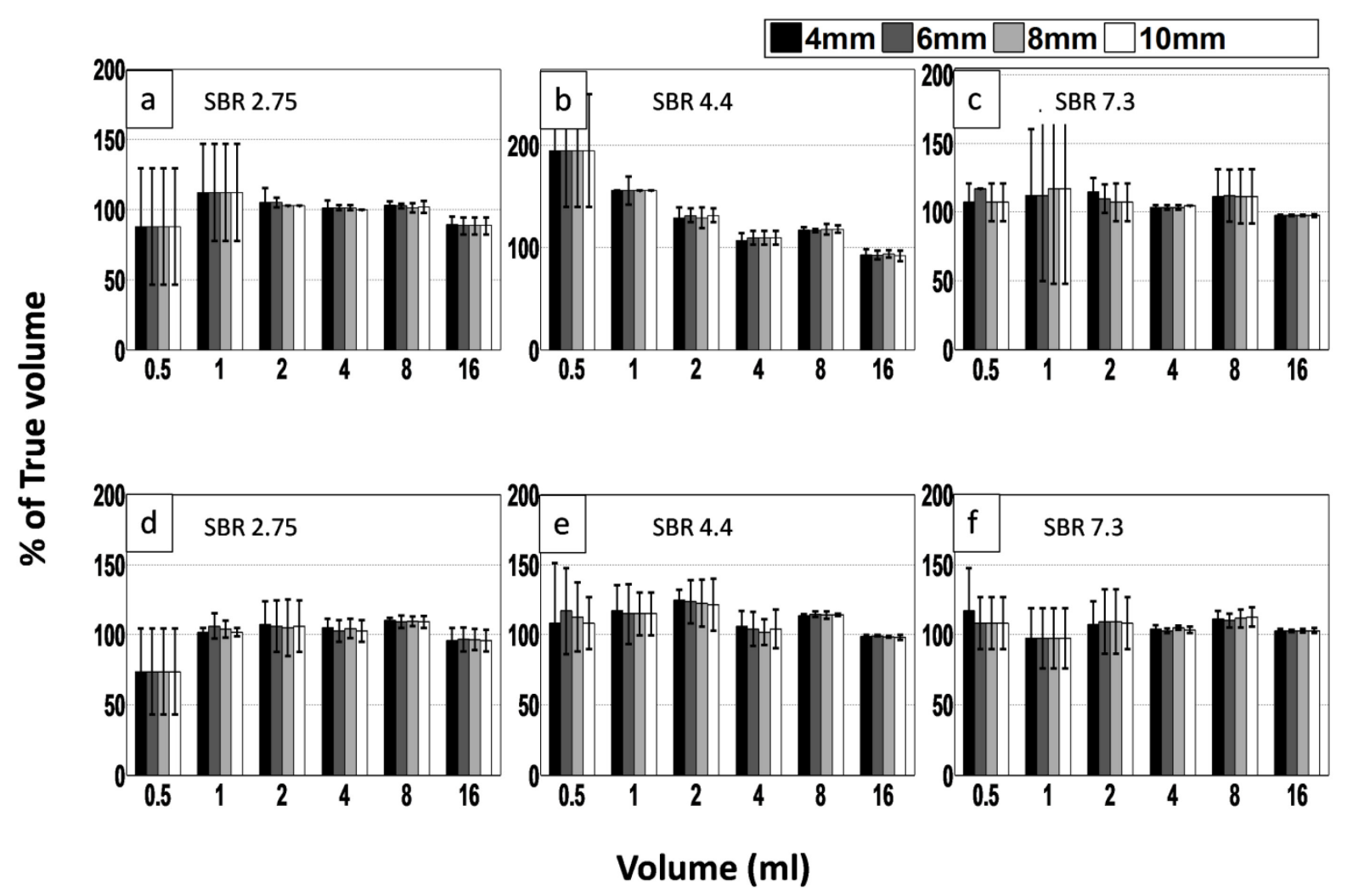

Figure $5.3 \mathrm{FV}$ for different SBR by changing FWHM ( $4 \mathrm{~mm}, 6 \mathrm{~mm}, 8 \mathrm{~mm}$ and $10 \mathrm{~mm})$. The FV in (a), (b) and (c) is for pixel size $5.46 \mathrm{~mm}$ and in (d), (e) and (f) pixel size is $3.64 \mathrm{~mm}$. The scan time is $5 \mathrm{~min}$ and matrix size is $5 \times 5$. Error bars correspond to standard deviation of the number of data sets $(\mathrm{n}=2)$. 


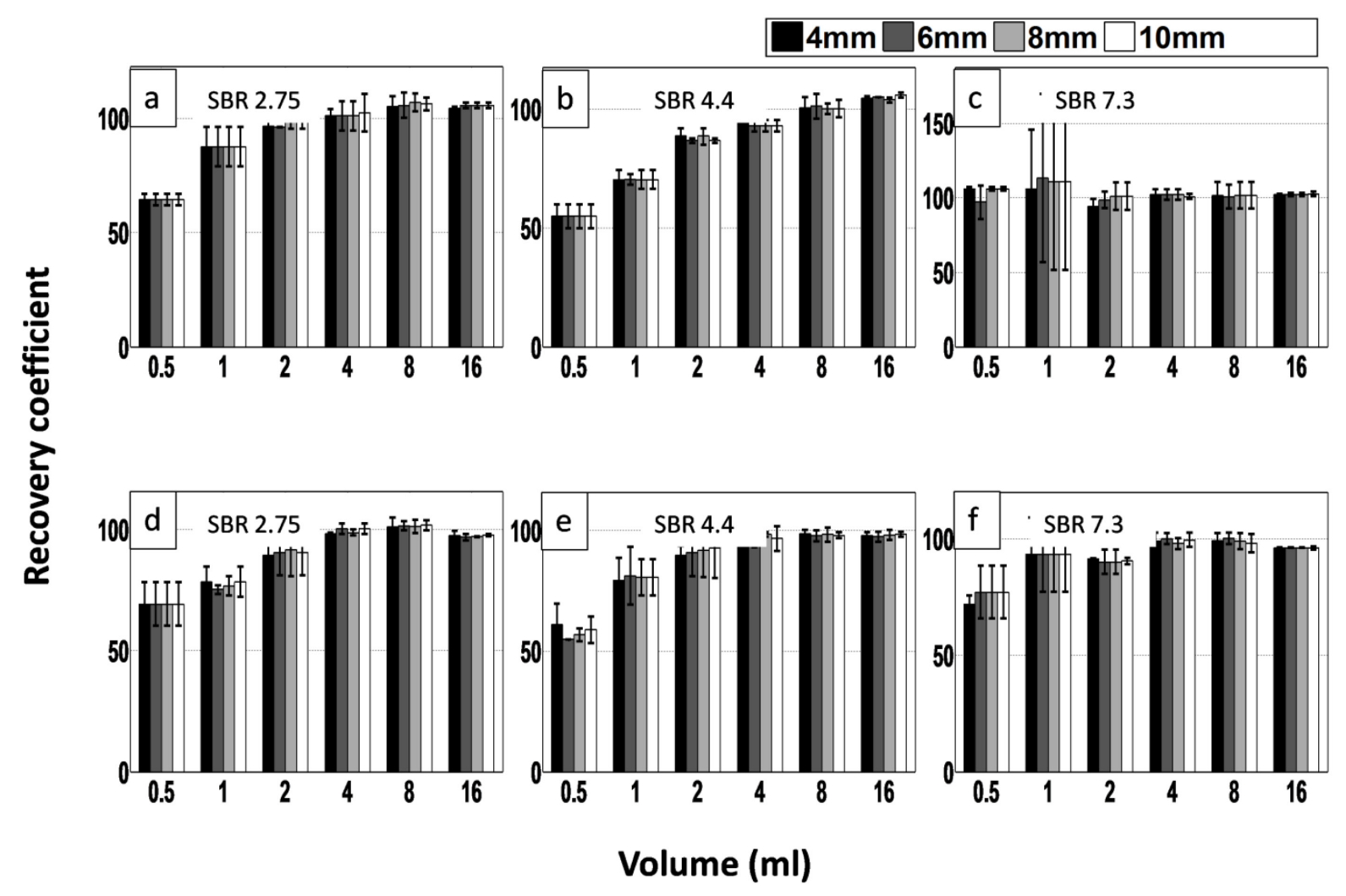

Figure 5.4 RC for different SBR by changing FWHM (4 mm, $6 \mathrm{~mm}, 8 \mathrm{~mm}$ and $10 \mathrm{~mm})$. The RC in (a), (b) and (c) is for pixel size $5.46 \mathrm{~mm}$ and in (d), (e) and (f) pixel size is $3.64 \mathrm{~mm}$. The scan time is $5 \mathrm{~min}$ and matrix size is $5 \times 5$. Error bars correspond to standard deviation of the number of data sets $(n=2)$. 


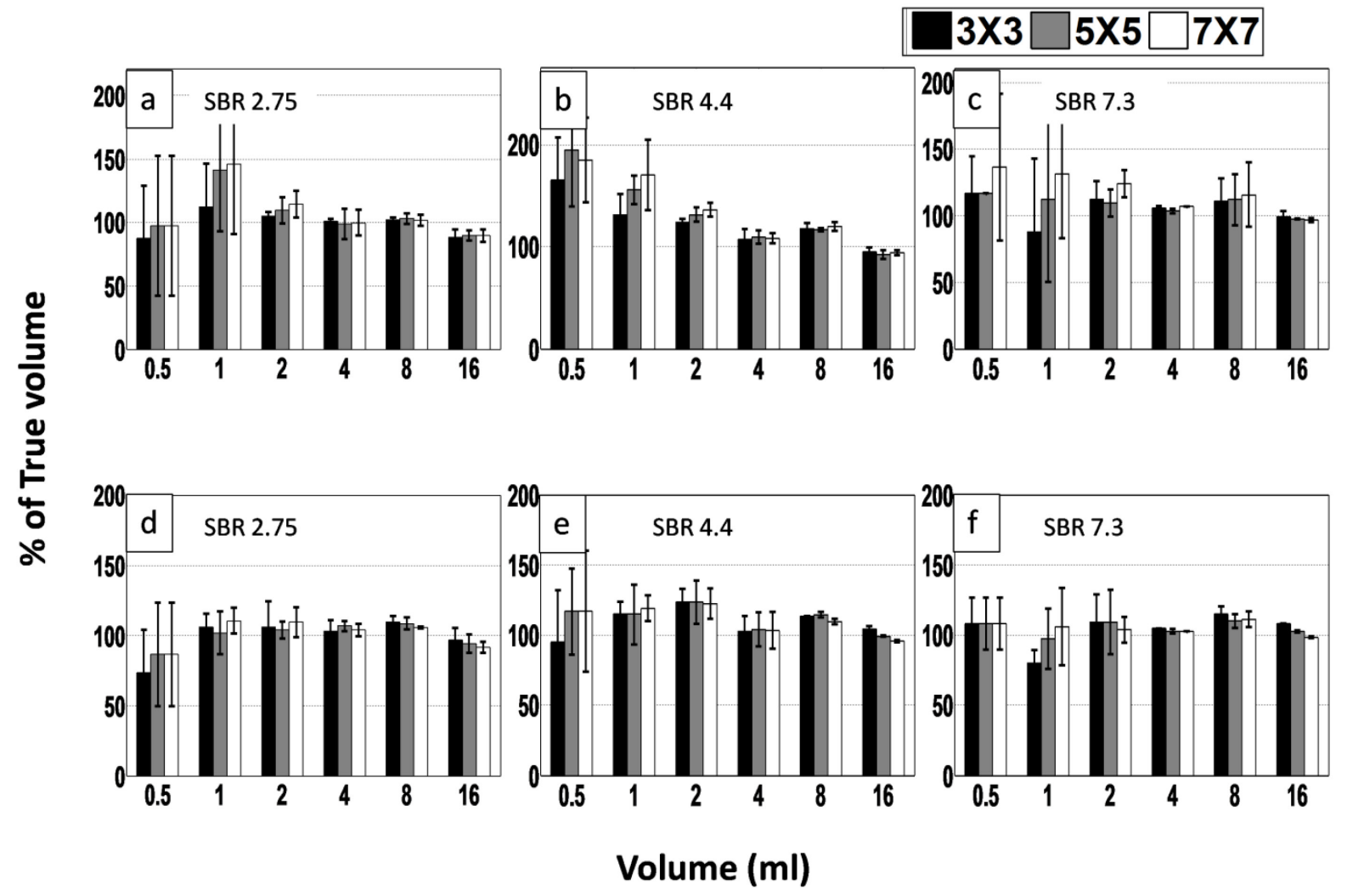

Figure 5.5 FV for different SBR by changing matrix size $(3 \times 3,5 \times 5$ and $7 \times 7)$. The FV in (a), (b) and (c) is for pixel size $5.46 \mathrm{~mm}$ and in (d), (e) and (f) pixel size is $3.64 \mathrm{~mm}$. The scan time is $5 \mathrm{~min}$ and FWHM is $6 \mathrm{~mm}$. Error bars correspond to standard deviation of the number of data sets $(n=2)$. 


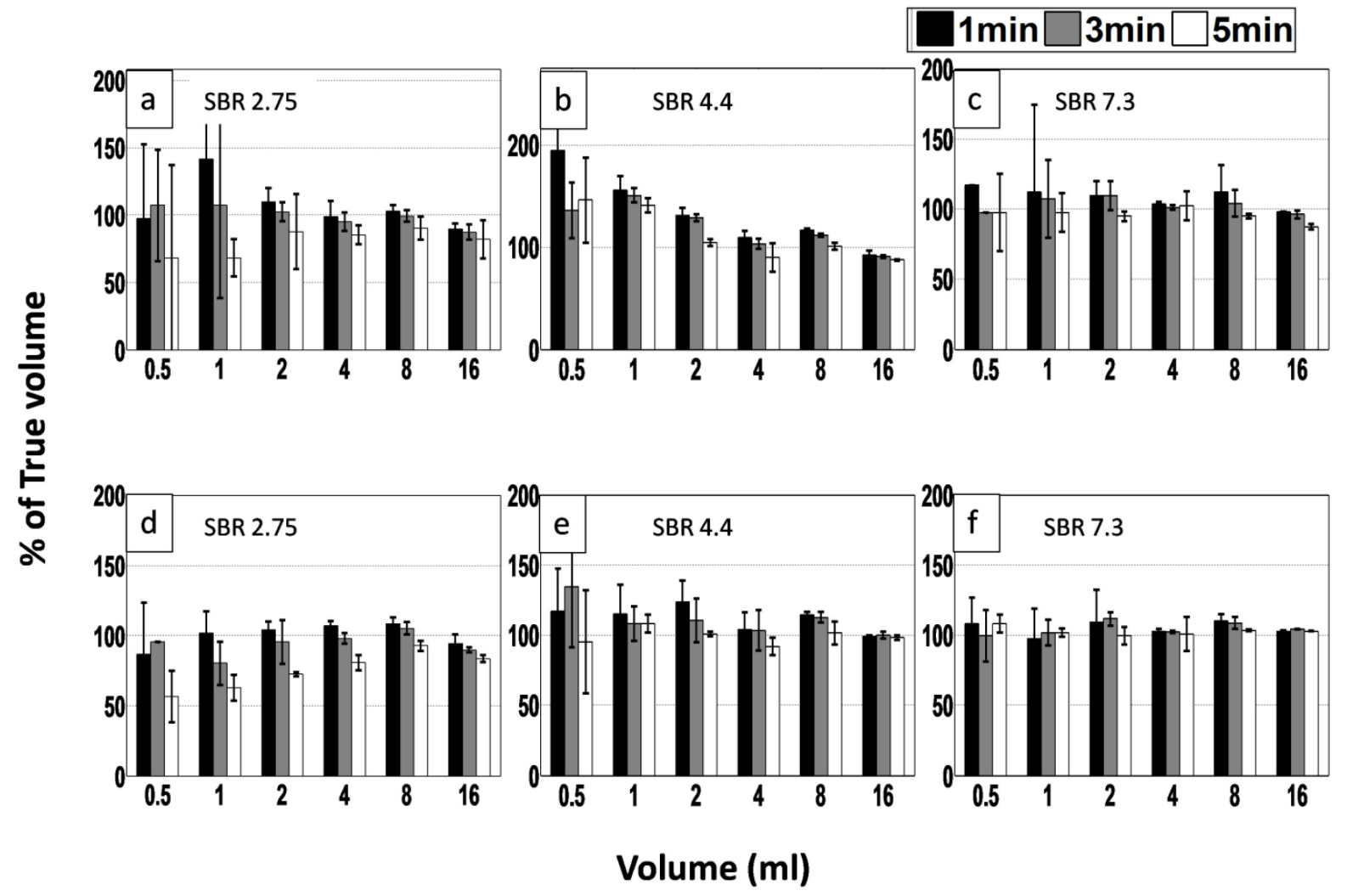

Figure 5.6 RC for different SBR by changing matrix size $(3 \times 3,5 \times 5$ and $7 \times 7)$. The $\mathrm{RC}$ in $(\mathrm{a})$, (b) and (c) is for pixel size $5.46 \mathrm{~mm}$ and in (d), (e) and (f) pixel size is $3.64 \mathrm{~mm}$. The scan time is $5 \mathrm{~min}$ and FWHM is $6 \mathrm{~mm}$. Error bars correspond to standard deviation of the number of data sets $(n=2)$. 


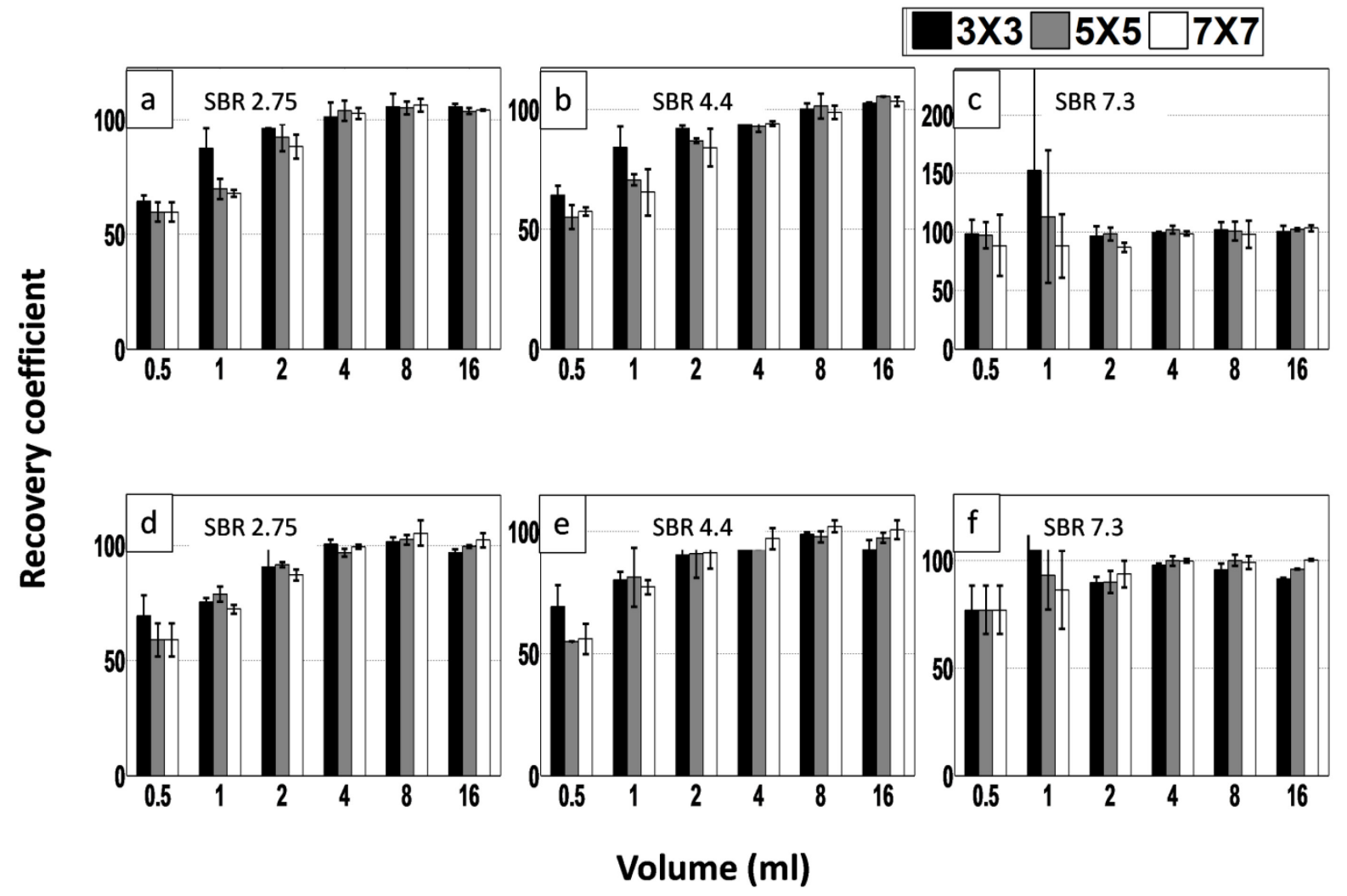

Figure 5.7 FV for different SBR by changing scan time (1 min, $3 \mathrm{~min}$ and $5 \mathrm{~min}$ ). The FV in (a), (b) and (c) is for pixel size $5.46 \mathrm{~mm}$ and in (d), (e) and (f) pixel size is $3.64 \mathrm{~mm}$. The FWHM is kept at $6 \mathrm{~mm}$ and matrix size is $5 \times 5$. Error bars correspond to standard deviation of the number of data sets $(n=2)$. 


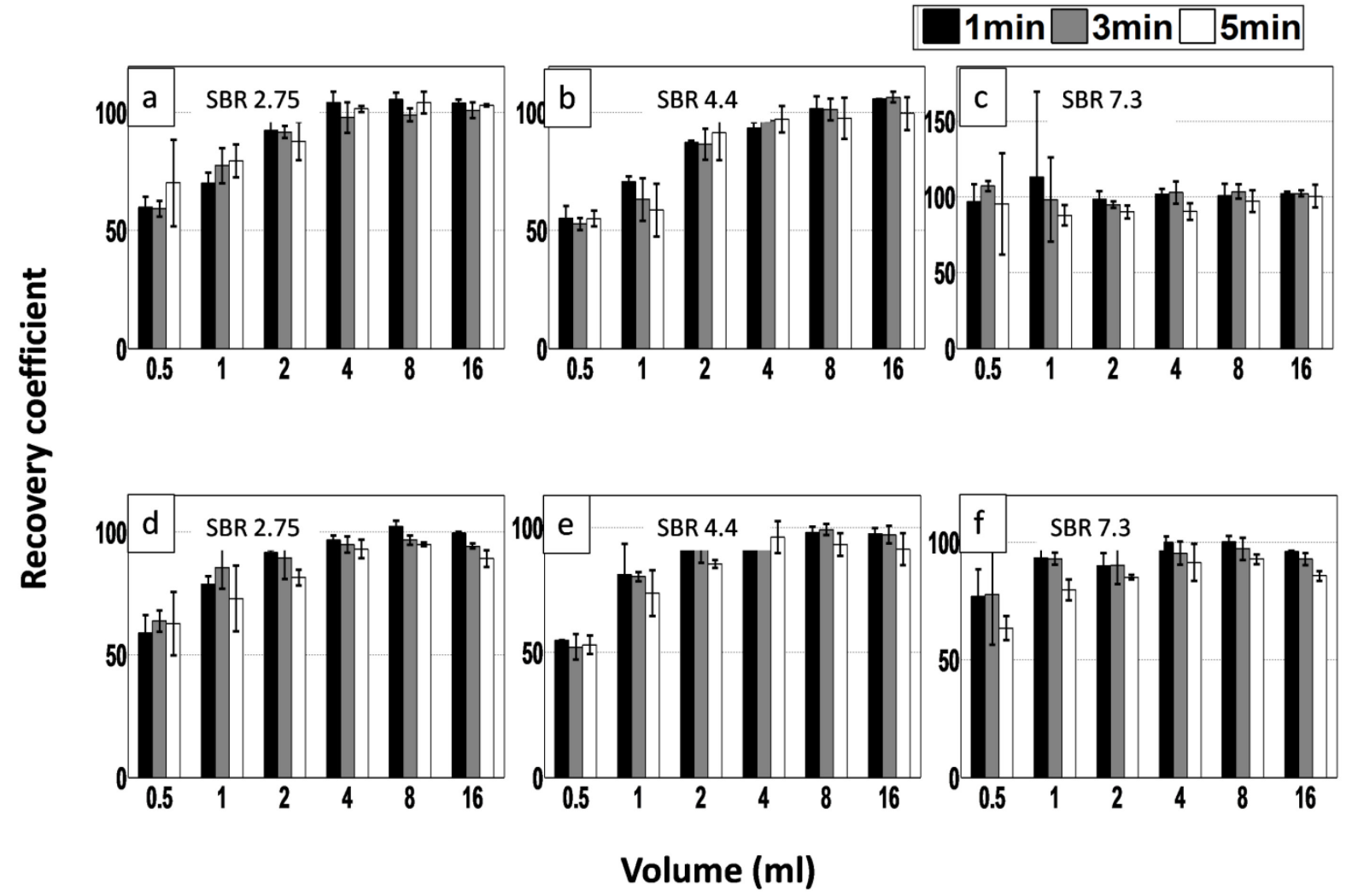

Figure 5.8 RC for different SBR by changing scan time (1 min, $3 \mathrm{~min}$ and $5 \mathrm{~min}$ ). The RC in (a), (b) and (c) is for pixel size $5.46 \mathrm{~mm}$ and in (d), (e) and (f) pixel size is $3.64 \mathrm{~mm}$. The FWHM is kept at $6 \mathrm{~mm}$ and matrix size is $5 \times 5$. Error bars correspond to standard deviation of the number of data sets $(n=2)$.

In Figure 5.3 the FWHM of the Gaussian PSF was changed to assume values of 4, 6, 8 and $10 \mathrm{~mm}$. The FWHM was not chosen lower than four because at the lower FWHM $(<4)$ the deconvolution algorithm is unable to remove the blur completely. For Figure 5.3, three levels of SBR were chosen $(2.75,4.4$ and 7.3$)$. The results shown are for a $5 \mathrm{~min}$ scan, and the matrix size of the PSF was kept at $5 \times 5$. The FV shown in Figure 5.3 was used to calculate the RC of the AC (Figure 5.4). The impact of the matrix size of the Gaussian PSF was tested by considering 
sizes of $3 \times 3,5 \times 5$ and $7 \times 7$. The FWHM and acquisition time were kept constant at $6 \mathrm{~mm}$ and 5 min, respectively. Figure 5.5 shows the FV and Figure 5.6 shows RC for different PSF matrix sizes. The algorithm performance was also tested by varying the acquisition time to observe the variation in FV and $\mathrm{RC}$ due to the different noise levels. FV and $\mathrm{RC}$ are shown in Figure 5.7 and Figure 5.8, respectively, with FWHM and matrix size of PSF kept constant. The approximate mid value among the range of FWHM and matrix size tested was chosen to be $6 \mathrm{~mm}$ and $5 \times 5$, respectively.

FV and RC results (Figures 5.3-5.8) were measured over multiple parameters. To make a better comparison of results from the proposed algorithm to those published in other literature, the FV and RC were plotted by keeping most of the parameters constant and varying only the tumor size and SBR. The parameters chosen to be kept constant were pixel size, FWHM, matrix size and acquisition time, whose values were $3.64 \mathrm{~mm}, 5 \times 5$ and $5 \mathrm{~min}$ respectively (Figures 5.8). The criterion for choosing the values of the above parameters was that they gave the best results for FV and RC.

\subsubsection{STATASTICAL ANALYSIS OF PHANTOM STUDIES}

The goal of this project was to develop a numerical algorithm that will provide accurate estimates of liver tumor Functional Volume (FV) and activity Recovery Coefficient (RC) with minimal input required from a physician or technologist, and further that it would be independent of the camera used to collect the images. To this end numerous parameters related to the quality of the image including noise (acquisition time), SBR (signal to background ratio), and parameters specific to the camera and reconstruction including FWHM (full width half max of the camera's point spread function), matrix size and image size were varied and their impact on FV and RC observed. From the graphs in Figures 5.3-5.8, 2 parameters were kept constant and 
the other 4 were changed so that the data could be more easily visualized on a 2-dimensional plot. However, this representation fails to provide a concise way to allow a clear understanding of which parameters are the major contributor to the variation in the results. To help in the understanding of the contribution of each parameter we designed a factorial analysis and measured the Effect value of each parameter. The analysis consisted of calculating the effects of each of the 6 factors in a statistical sense. The analysis is based on the physical phantom data of homogeneous tumors with two replicates and is presented in the next section.

\subsection{6.a Factorial analysis}

The statistical analysis allows the testing of the hypothesis that the performance of the algorithm is independent of the camera used to generate the images. There are in total six parameters (factors) which were changed, as listed in Table 5.1. The two outputs or response variables are FV and RC. The levels of the factors are also given in the table below.

Table 5.1 Parameters (factors) and their values (levels) used in the factorial analysis. All factor levels are treated as nominal data.

\begin{tabular}{|c|c|c|c|c|c|c|}
\hline Image size & $5.46 \mathrm{~mm}$ & $3.64 \mathrm{~mm}$ & & & & \\
\hline Acquisition time & $1 \mathrm{~min}$ & $3 \mathrm{~min}$ & $5 \mathrm{~min}$ & & & \\
\hline Signal to background ratio & 2.75 & 4.4 & 8.8 & & & \\
\hline Matrix size & $3 \times 3$ & $5 \times 5$ & $7 \times 7$ & & & \\
\hline FWHM & $4 \mathrm{~mm}$ & $6 \mathrm{~mm}$ & $8 \mathrm{~mm}$ & $10 \mathrm{~mm}$ & & \\
\hline Tumor volume & $0.5 \mathrm{ml}$ & $1 \mathrm{ml}$ & $2 \mathrm{ml}$ & $4 \mathrm{ml}$ & $8 \mathrm{ml}$ & $16 \mathrm{ml}$ \\
\hline
\end{tabular}


The Effect values were calculated using the software Design Expert 8.0. The Image size is the size of each pixel in the image. Acquisition time is related to noise and thus shorter scanning time equates to higher noise and longer scanning time equates to lower noise. Three levels of signal to background ratio (SBR) are used. Matrix size is the size of the point spread function (PSF) and full width half maximum (FWHM) of the PSF. Tumor volume is the known volume of spheres. The Effect values and p-values were calculated for each parameter and are listed in Table 5.2.

Figure 5.9 is a plot of the Effect values for each Tumor volume. In Figure 5.9.a the response variable is FV and in Figure 5.9.b the response variable is RC. Figure 5.9 shows that the FWHM has no significant impact on the FV and RC. It can also be observes that the Image Size has a significant impact on FV and RC for the $16 \mathrm{ml}$ Tumor. Image Size was changed post reconstruction and its impact on other parameters cannot be known from the current statistics. It is known from the literature that the tumor size has the most impact on the RC, which is also confirmed by our statistical analysis and shown in Figure 5.10.b. SBR and Acquisition Time are also known to have significant impact on the FV and RC measurement and is also confirmed by the analysis of our algorithm. These finding are not surprising, but the statistical analysis provides an appreciation of the degree of contribution of these factors on the algorithm's performance.

Figure 5.10 is a plot of the Effect values of all 6 parameters (factors), including tumor size, for the two response variables FV (Figure 5.10.a) and RC (Figure 5.10.b). The FWHM has no impact on the FV and RC, and Matrix Size has little effect, except for perhaps the largest tumor. This finding supports the hypothesis that the algorithm is independent of the camera used to collect the images and suggests that the algorithm can confidently be applied on images 
acquired from different cameras. Further, the statistics prove that the algorithm could accurately perform PVE correction without knowing the spatial resolution of the camera.

There is an interesting amplification of the effect of Image Size on FV and RC that is difficult to explain without further investigation. It could be that the accuracy and precision of the FV and $\mathrm{RC}$ estimates for the largest tumor was such that high statistical significance was obtained, while there may be little practical significance. A more in depth analysis of these results still must be done.

The Effect values due to the interaction of different parameters (factors) are shown in Table 5.2 and Table 5.3 for response variable FV and RC respectively. The primary purpose of doing this statistical analysis was to show that FWHM has no impact on the FV and RC. We also wanted to observe the best parameter value that would provide an accurate estimation of FV and RC. The current statistical study is unable to provide the best parameters because the factor level values are nominal rather than numerical. This precludes a quantitative model fit. To find the optimal parameters, the statistics would have to be done for each sub levels of the parameter and this could be further investigated. This was outside the scope of this dissertation. 


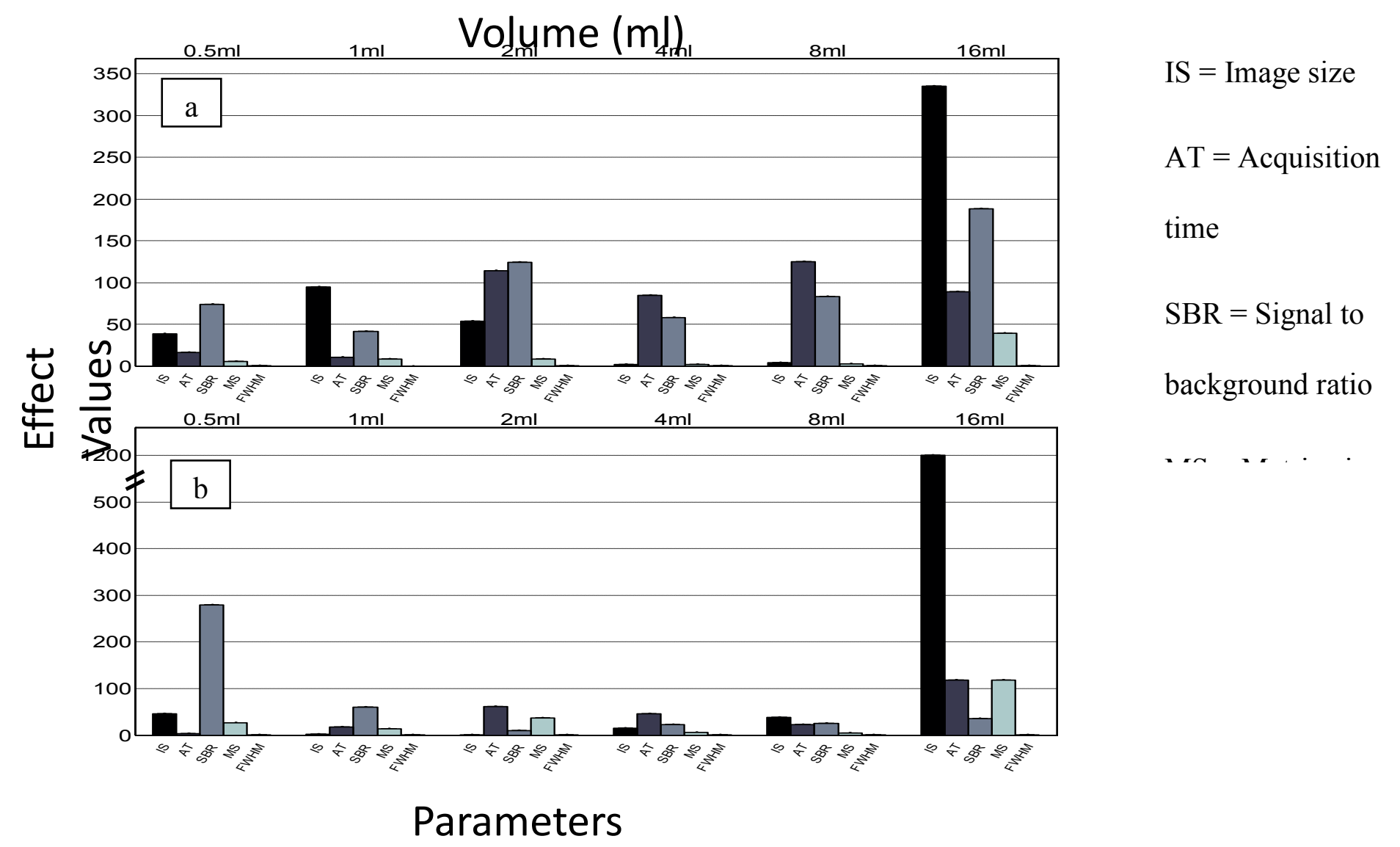

Figure 5.9 Contribution of different parameter in terms of effect values in measurement of (a) FV and (b) RC for different size tumors. 

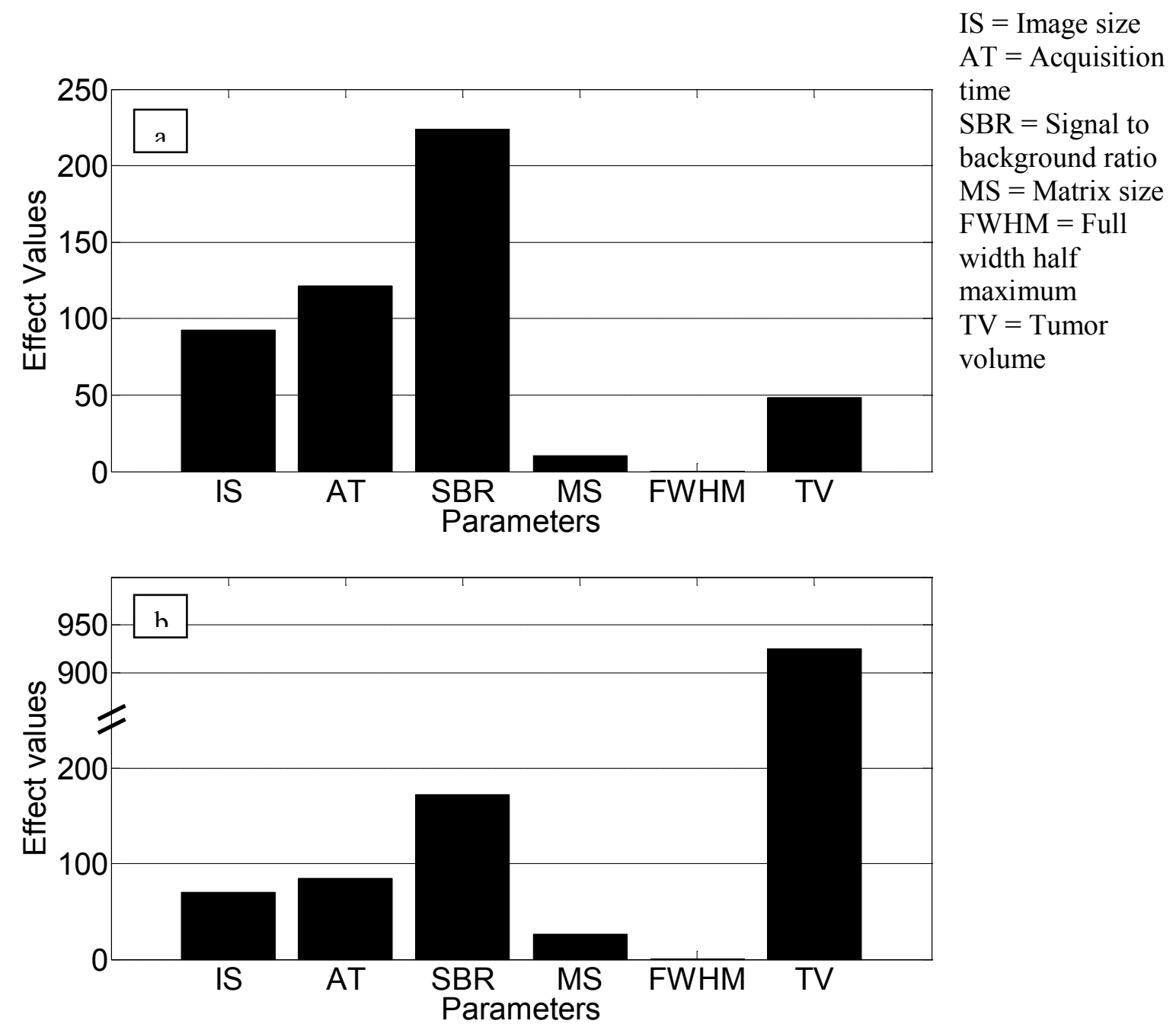

Figure 5.10 Contribution of different parameters in term of Effect values for measuring (a) FV and (b) RC considering true volume (TV) as one of the parameter. 
Table 5.2 Effect values of each of the parameters (factors) and their corresponding interaction for the response variable $\mathrm{FV}$. $(\mathrm{TV}=$ tumor volume, $\mathrm{IS}=$ image size, $\mathrm{AT}=$ aqusition time, $\mathrm{SBR}=$ signal to background ratio, $\mathrm{MS}=$ matrix size and $\mathrm{FWHM}=$ full width half maximum)

\begin{tabular}{|l|r|r|r|r|r|r|}
\hline \multicolumn{1}{|c|}{ Term } & \multicolumn{1}{|c|}{$\mathrm{df}$} & SumSqr & MeanSqr & Prob $>\mathrm{F}$ & $\mathrm{F}$ Value & \%Contribution \\
\hline A-TV & 5 & 10.36352 & 2.072704 & $<0.0001$ & 48.42853 & 7.290664 \\
\hline B-IS & 1 & 3.964439 & 3.964439 & $<0.0001$ & 92.62873 & 2.788955 \\
\hline C-AT & 2 & 10.40554 & 5.202771 & $<0.0001$ & 121.5622 & 7.320225 \\
\hline D-SBR & 2 & 19.17799 & 9.588996 & $<0.0001$ & 224.0459 & 13.49158 \\
\hline E-MS & 2 & 0.890347 & 0.445173 & $<0.0001$ & 10.40143 & 0.626352 \\
\hline F- & & & & & & \\
FWHM & 3 & 0.003339 & 0.001113 & 0.9943 & 0.026004 & 0.002349 \\
\hline AB & 5 & 9.30996 & 1.861992 & $<0.0001$ & 43.50526 & 6.54949 \\
\hline AC & 10 & 1.508862 & 0.150886 & 0.0001 & 3.525441 & 1.061474 \\
\hline AD & 10 & 10.81621 & 1.081621 & $<0.0001$ & 25.27197 & 7.609127 \\
\hline AE & 10 & 2.072835 & 0.207283 & $<0.0001$ & 4.843157 & 1.458224 \\
\hline AF & 15 & 0.006004 & 0.0004 & 1.0000 & 0.009352 & 0.004223 \\
\hline & 2 & 0.271572 & 0.135786 & 0.0422 & 3.172621 & 0.191049 \\
\hline & & & & & & \\
\hline & & & & & & \\
\hline
\end{tabular}




\begin{tabular}{|c|c|c|c|c|c|c|}
\hline$B D$ & 2 & 1.621757 & 0.810879 & $<0.0001$ & 18.9461 & 1.140895 \\
\hline $\mathrm{BE}$ & 2 & 0.515191 & 0.257595 & 0.0025 & 6.018691 & 0.362433 \\
\hline $\mathrm{BF}$ & 3 & 0.00019 & $6.34 \mathrm{E}-05$ & 0.9999 & 0.001482 & 0.000134 \\
\hline$C D$ & 4 & 1.604313 & 0.401078 & $<0.0001$ & 9.371155 & 1.128623 \\
\hline $\mathrm{CE}$ & 4 & 0.15191 & 0.037977 & 0.4707 & 0.887339 & 0.106867 \\
\hline CF & 6 & 0.003209 & 0.000535 & 1.0000 & 0.012496 & 0.002257 \\
\hline $\mathrm{DE}$ & 4 & 0.15433 & 0.038583 & 0.4623 & 0.901478 & 0.10857 \\
\hline DF & 6 & 0.002957 & 0.000493 & 1.0000 & 0.011515 & 0.00208 \\
\hline $\mathrm{EF}$ & 6 & 0.010082 & 0.00168 & 0.9997 & 0.039261 & 0.007093 \\
\hline$A B C$ & 10 & 1.302063 & 0.130206 & 0.0008 & 3.042257 & 0.915992 \\
\hline$A B D$ & 10 & 3.226012 & 0.322601 & $<0.0001$ & 7.537544 & 2.269476 \\
\hline$A B E$ & 10 & 0.401495 & 0.040149 & 0.4968 & 0.938089 & 0.282449 \\
\hline$A B F$ & 15 & 0.009914 & 0.000661 & 1.0000 & 0.015442 & 0.006974 \\
\hline ACD & 20 & 2.767085 & 0.138354 & $<0.0001$ & 3.232633 & 1.946624 \\
\hline ACE & 20 & 0.4242 & 0.02121 & 0.9692 & 0.49557 & 0.298422 \\
\hline ACF & 30 & 0.015153 & 0.000505 & 1.0000 & 0.011802 & 0.01066 \\
\hline ADE & 20 & 0.552446 & 0.027622 & 0.8802 & 0.645392 & 0.388641 \\
\hline
\end{tabular}




\begin{tabular}{|c|c|c|c|c|c|c|}
\hline ADF & 30 & 0.027352 & 0.000912 & 1.0000 & 0.021302 & 0.019242 \\
\hline AEF & 30 & 0.02769 & 0.000923 & 1.0000 & 0.021566 & 0.01948 \\
\hline $\mathrm{BCD}$ & 4 & 0.717984 & 0.179496 & 0.0022 & 4.193908 & 0.505097 \\
\hline BCE & 4 & 0.058014 & 0.014503 & 0.8518 & 0.338872 & 0.040812 \\
\hline $\mathrm{BCF}$ & 6 & 0.004734 & 0.000789 & 1.0000 & 0.018436 & 0.00333 \\
\hline $\mathrm{BDE}$ & 4 & 0.222685 & 0.055671 & 0.2677 & 1.300752 & 0.156657 \\
\hline BDF & 6 & 0.006575 & 0.001096 & 0.9999 & 0.025602 & 0.004625 \\
\hline BEF & 6 & 0.010331 & 0.001722 & 0.9997 & 0.040229 & 0.007268 \\
\hline CDE & 8 & 0.009491 & 0.001186 & 1.0000 & 0.027718 & 0.006677 \\
\hline CDF & 12 & 0.017419 & 0.001452 & 1.0000 & 0.033915 & 0.012254 \\
\hline CEF & 12 & 0.012921 & 0.001077 & 1.0000 & 0.025158 & 0.00909 \\
\hline DEF & 12 & 0.017133 & 0.001428 & 1.0000 & 0.033359 & 0.012053 \\
\hline$A B C D$ & 20 & 2.066249 & 0.103312 & 0.0005 & 2.413885 & 1.453591 \\
\hline ABCE & 20 & 0.224474 & 0.011224 & 0.9996 & 0.262241 & 0.157916 \\
\hline $\mathrm{ABCF}$ & 30 & 0.023025 & 0.000768 & 1.0000 & 0.017933 & 0.016198 \\
\hline$A B D E$ & 20 & 0.333605 & 0.01668 & 0.9930 & 0.389733 & 0.234689 \\
\hline ABDF & 30 & 0.031553 & 0.001052 & 1.0000 & 0.024575 & 0.022198 \\
\hline
\end{tabular}




\begin{tabular}{|c|c|c|c|c|c|c|}
\hline ABEF & 30 & 0.019473 & 0.000649 & 1.0000 & 0.015166 & 0.013699 \\
\hline ACDE & 40 & 0.165647 & 0.004141 & 1.0000 & 0.096758 & 0.116532 \\
\hline ACDF & 60 & 0.053631 & 0.000894 & 1.0000 & 0.020885 & 0.037729 \\
\hline ACEF & 60 & 0.099695 & 0.001662 & 1.0000 & 0.038823 & 0.070135 \\
\hline ADEF & 60 & 0.048004 & 0.0008 & 1.0000 & 0.018694 & 0.033771 \\
\hline $\mathrm{BCDE}$ & 8 & 0.074447 & 0.009306 & 0.9879 & 0.21743 & 0.052373 \\
\hline $\mathrm{BCDF}$ & 12 & 0.016833 & 0.001403 & 1.0000 & 0.032775 & 0.011842 \\
\hline BCEF & 12 & 0.007942 & 0.000662 & 1.0000 & 0.015464 & 0.005587 \\
\hline BDEF & 12 & 0.013409 & 0.001117 & 1.0000 & 0.026108 & 0.009433 \\
\hline CDEF & 24 & 0.028056 & 0.001169 & 1.0000 & 0.027314 & 0.019737 \\
\hline$A B C D E$ & 40 & 0.39272 & 0.009818 & 1.0000 & 0.229397 & 0.276276 \\
\hline$A B C D F$ & 60 & 0.06466 & 0.001078 & 1.0000 & 0.02518 & 0.045488 \\
\hline ABCEF & 60 & 0.043165 & 0.000719 & 1.0000 & 0.016809 & 0.030366 \\
\hline ABDEF & 60 & 0.049533 & 0.000826 & 1.0000 & 0.019289 & 0.034846 \\
\hline ACDEF & 120 & 0.140967 & 0.001175 & 1.0000 & 0.027447 & 0.099169 \\
\hline BCDEF & 24 & 0.027291 & 0.001137 & 1.0000 & 0.026569 & 0.019199 \\
\hline ABCDEF & 120 & 0.113198 & 0.000943 & 1.0000 & 0.022041 & 0.079634 \\
\hline
\end{tabular}


Table 5.3 Effect values of each of the parameters (factors) and their corresponding interaction for the response variable $\mathrm{RC}$. $(\mathrm{TV}=$ tumor volume, $\mathrm{IS}=$ image size, $\mathrm{AT}=$ aqusition time, $\mathrm{SBR}=$ signal to background ratio, $\mathrm{MS}=$ matrix size and $\mathrm{FWHM}=$ full width half maximum)

\begin{tabular}{|c|c|c|c|c|c|c|}
\hline Term & $d f$ & SumSqr & MeanSqr & Prob $>F$ & F Value & \% Contribution \\
\hline A-TV & 5 & 35.33211 & 7.066422 & $<0.0001$ & 958.7613 & 54.90496 \\
\hline B-IS & 1 & 0.517484 & 0.517484 & $<0.0001$ & 70.21148 & 0.804154 \\
\hline C-AT & 2 & 1.252574 & 0.626287 & $<0.0001$ & 84.97365 & 1.946459 \\
\hline D-SBR & 2 & 2.540063 & 1.270032 & $<0.0001$ & 172.3159 & 3.947176 \\
\hline E-MS & 2 & 0.385528 & 0.192764 & $<0.0001$ & 26.15396 & 0.599099 \\
\hline F-FWHM & 3 & 0.003377 & 0.001126 & 0.9280 & 0.152713 & 0.005247 \\
\hline$A B$ & 5 & 0.813919 & 0.162784 & $<0.0001$ & 22.08626 & 1.264804 \\
\hline$A C$ & 10 & 0.38386 & 0.038386 & $<0.0001$ & 5.20815 & 0.596506 \\
\hline$A D$ & 10 & 5.75199 & 0.575199 & $<0.0001$ & 78.04212 & 8.938407 \\
\hline $\mathrm{AE}$ & 10 & 1.142252 & 0.114225 & $<0.0001$ & 15.49791 & 1.775023 \\
\hline $\mathrm{AF}$ & 15 & 0.011033 & 0.000736 & 1.0000 & 0.099797 & 0.017145 \\
\hline$B C$ & 2 & 0.036251 & 0.018125 & 0.0859 & 2.459226 & 0.056333 \\
\hline
\end{tabular}




\begin{tabular}{|l|r|r|r|l|l|l|}
\hline BD & 2 & 0.770782 & 0.385391 & $<0.0001$ & 52.28929 & 1.197771 \\
\hline BE & 2 & 0.2767 & 0.13835 & $<0.0001$ & 18.7711 & 0.429983 \\
\hline BF & 3 & 0.000982 & 0.000327 & 0.9876 & 0.044424 & 0.001526 \\
\hline CD & 4 & 0.406171 & 0.101543 & $<0.0001$ & 13.77718 & 0.631177 \\
\hline CE & 4 & 0.062159 & 0.01554 & 0.0776 & 2.108421 & 0.096594 \\
\hline CF & 6 & 0.004955 & 0.000826 & 0.9951 & 0.11204 & 0.007699 \\
\hline DE & 4 & 0.098234 & 0.024558 & 0.0100 & 3.332052 & 0.152652 \\
\hline DF & 6 & 0.00456 & 0.00076 & 0.9961 & 0.103125 & 0.007087 \\
\hline EF & 6 & 0.010153 & 0.001692 & 0.9671 & 0.229579 & 0.015777 \\
\hline ABC & 10 & 0.272328 & 0.027233 & $<0.0001$ & 3.694905 & 0.423189 \\
\hline ABD & 10 & 0.813934 & 0.081393 & $<0.0001$ & 11.04333 & 1.264828 \\
\hline ABE & 10 & 0.33035 & 0.033035 & $<0.0001$ & 4.482143 & 0.513354 \\
\hline ABF & 15 & 0.007522 & 0.000501 & 1.0000 & 0.068036 & 0.011689 \\
\hline ACD & 20 & 0.628203 & 0.03141 & $<0.0001$ & 4.261679 & 0.976207 \\
\hline ACF & 20 & 0.222477 & 0.011124 & 0.0691 & 1.509269 & 0.345723 \\
\hline & 30 & 0.017588 & 0.000586 & 1.0000 & 0.079545 & 0.027331 \\
\hline & 20 & 0.40855 & 0.020427 & $<0.0001$ & 2.771571 & 0.634873 \\
\hline & & & & & & \\
\hline & & & & & & \\
\hline
\end{tabular}




\begin{tabular}{|c|c|c|c|c|c|c|}
\hline ADF & 30 & 0.030158 & 0.001005 & 1.0000 & 0.136393 & 0.046864 \\
\hline $\mathrm{AEF}$ & 30 & 0.035721 & 0.001191 & 1.0000 & 0.161554 & 0.05551 \\
\hline$B C D$ & 4 & 0.220091 & 0.055023 & $<0.0001$ & 7.465398 & 0.342014 \\
\hline BCE & 4 & 0.032009 & 0.008002 & 0.3621 & 1.085746 & 0.049742 \\
\hline$B C F$ & 6 & 0.001124 & 0.000187 & 0.9999 & 0.025415 & 0.001747 \\
\hline BDE & 4 & 0.175191 & 0.043798 & $<0.0001$ & 5.942414 & 0.272241 \\
\hline BDF & 6 & 0.005289 & 0.000882 & 0.9941 & 0.119606 & 0.008219 \\
\hline BEF & 6 & 0.014567 & 0.002428 & 0.9217 & 0.329406 & 0.022637 \\
\hline $\mathrm{CDE}$ & 8 & 0.028795 & 0.003599 & 0.8651 & 0.488359 & 0.044747 \\
\hline CDF & 12 & 0.008226 & 0.000686 & 1.0000 & 0.093008 & 0.012783 \\
\hline CEF & 12 & 0.009252 & 0.000771 & 0.9999 & 0.104608 & 0.014377 \\
\hline DEF & 12 & 0.02196 & 0.00183 & 0.9956 & 0.248291 & 0.034125 \\
\hline$A B C D$ & 20 & 0.299555 & 0.014978 & 0.0046 & 2.032155 & 0.465498 \\
\hline$A B C E$ & 20 & 0.144571 & 0.007229 & 0.4830 & 0.980761 & 0.224659 \\
\hline$A B C F$ & 30 & 0.015998 & 0.000533 & 1.0000 & 0.072352 & 0.02486 \\
\hline ABDE & 20 & 0.157992 & 0.0079 & 0.3735 & 1.071803 & 0.245514 \\
\hline ABDF & 30 & 0.020253 & 0.000675 & 1.0000 & 0.091598 & 0.031473 \\
\hline
\end{tabular}




\begin{tabular}{|c|c|c|c|c|c|c|}
\hline ABEF & 30 & 0.026842 & 0.000895 & 1.0000 & 0.121395 & 0.041711 \\
\hline ACDE & 40 & 0.188262 & 0.004707 & 0.9617 & 0.638579 & 0.292554 \\
\hline ACDF & 60 & 0.048117 & 0.000802 & 1.0000 & 0.108808 & 0.074773 \\
\hline ACEF & 60 & 0.055392 & 0.000923 & 1.0000 & 0.125259 & 0.086078 \\
\hline ADEF & 60 & 0.064556 & 0.001076 & 1.0000 & 0.145982 & 0.100319 \\
\hline $\mathrm{BCDE}$ & 8 & 0.059716 & 0.007464 & 0.4242 & 1.01277 & 0.092797 \\
\hline BCDF & 12 & 0.003856 & 0.000321 & 1.0000 & 0.043596 & 0.005992 \\
\hline BCEF & 12 & 0.009261 & 0.000772 & 0.9999 & 0.104709 & 0.014391 \\
\hline BDEF & 12 & 0.018181 & 0.001515 & 0.9983 & 0.205564 & 0.028253 \\
\hline CDEF & 24 & 0.021849 & 0.00091 & 1.0000 & 0.123518 & 0.033953 \\
\hline$A B C D E$ & 40 & 0.280387 & 0.00701 & 0.5587 & 0.951062 & 0.435713 \\
\hline$A B C D F$ & 60 & 0.033937 & 0.000566 & 1.0000 & 0.076743 & 0.052738 \\
\hline ABCEF & 60 & 0.036593 & 0.00061 & 1.0000 & 0.082747 & 0.056864 \\
\hline ABDEF & 60 & 0.042102 & 0.000702 & 1.0000 & 0.095205 & 0.065425 \\
\hline ACDEF & 120 & 0.088019 & 0.000733 & 1.0000 & 0.099519 & 0.136779 \\
\hline BCDEF & 24 & 0.026869 & 0.00112 & 1.0000 & 0.151897 & 0.041753 \\
\hline$A B C D E F$ & 120 & 0.07599 & 0.000633 & 1.0000 & 0.085918 & 0.118086 \\
\hline
\end{tabular}




\subsubsection{DISCUSSION}

The main purpose of the proposed algorithm is to give accurate FV estimates and 3D contour of tumors which would help in the determination of the appropriate radiation dose for radiotherapy (e.g Y-90 microspheres SIRT, IMRT and radio-immunotherapy). The AC and Total Lesion Glycolysis (TLG) are also very useful in monitoring response to therapy, and TLG relies on accurate FV estimates. The algorithm described in this study is robust in that the shape and size of the user-defined ROI has no impact on the accuracy of the FV, as long as the ROI is within the organ of interest. Similarly, for a phantom experiment, the ROI must be positioned within the phantom. The contour of the ROI must be smaller than the size of the body because the HT algorithm requires the generation of two histograms, one for ROI and another for background. If the ROI is too

large then there would be too few pixels in the background and a very small background region compared to the ROI which would not be adequate enough to sample the entire spectrum of the background (Figure 5.1. h). This would cause the HT algorithm to incorrectly calculate the threshold value.

Li et al. used a Region Growing algorithm to achieve the segment similar to the HT algorithm [31]. In the Li et al. algorithm the identification of maximum intensity was used as an initiation point for the algorithm. An advantage of the proposed algorithm compared to that of Li et al. is that it can segment multiple tumors within a given ROI since the HT algorithm is not based on maximum intensity. The HT algorithm, in some slices, segments isolated pixels or isolated clusters which are not part of any of the 
tumors, due to the noisy nature of PET images. These isolated pixels can be easily removed by using region labeling.

Blur is removed from the segment $(S G)$ using the IDTS algorithm. The maximum intensity value $(M V)$ within the tumor is used in one of the stopping conditions to reach accurate volume estimation (Equation 3.15). The maximum intensity has been typically used to measure $\mathrm{SUV}_{\max }$ and is least affected by PVE [15]. The maximum intensity of an image could be affected by noise, but the algorithm was tested for different noise levels by collecting images at different acquisition times (Figures 5.7 and 5.8). Additionally, the MIC algorithm was used to correct the maximum intensity for tumors smaller than $1.8 \mathrm{ml}$. The algorithm chooses the tumor on which the MIC algorithm can be applied automatically. To do this the algorithm measures the FV of each tumor and if it finds any tumor whose volume is less than $1.8 \mathrm{ml}$ the MIC algorithm is applied on that particular tumor and the FV is recalculated.

Hatt et al. have been able to show high accuracy in estimating FV but they are not able to identify FV of tumors smaller than a diameter of $10 \mathrm{~mm}$ [44]. In contrast, our algorithm is able to segment and calculate the FV of tumors less than $10 \mathrm{~mm}$. Apart from FV estimation; the algorithm is also able to measure the activity concentration of the tumor. The purpose of the development of this algorithm is to segment tumors, while at the same time estimate FV and measure PVE corrected activity concentration (AC).

Kirov et al. and Teo et al. have successfully corrected the PVE using deconvolution $[49,50]$. Both methods require estimation of the PSF of the camera. Barbee et al. used a non-isotropic PSF which was estimated by fitting the blur to a 
Gaussian function [14]. But the PSF was measured by doing phantom studies where a cylinder filled with ${ }^{18} \mathrm{~F}$-FDG within the phantom was used as a point source while no activity was in the remainder of the phantom resulting in a very favorable SBR. The amount of blur in a tumor is also dependent on the SBR so it would be difficult to predefine the PSF [15]. In the cases of Kirov et al. and Teo et al. the number of iterations of the deconvolution algorithm applied had to be optimized based on phantom studies [49, 50]. In our algorithm the FV and PVE corrected RC are independent of the PSF used in the deconvolution algorithm and thus there was no need to optimize the number of iterations to get the final FV.

The value of FV is dependent on SG volume. In Figure 5.2 the variation in SG volume at SBR 2.75 leads to high variation in FV, as seen in Figure 5.3.a. This variation in SG volume could be due to the noise level in the image, which affects the HT algorithm for small tumors $(<2 \mathrm{ml})$ the most. In Figure 5.3.c the FV is closer to the actual volume at SBR 7.3, with minimal variation compared at SBR 2.75. Accurate FV leads to accurate RC as seen in Figure 5.4.c. Figures 5.3 and 5.4 show that the variation in FV and RC due to the use of different FWHMs in the algorithm is minimal. However, the size of the matrix of the PSF shows variation in FV and RC for small tumors $(<2 \mathrm{ml})$. The matrix sizes of $3 \times 3$ and $5 \times 5$ give better FV and RC compared to matrix size of $7 \times$ 7. However, for large tumors the matrix size shows minimal variation (Figures 5.5 and 5.6). Hence in any further application of the IDTS algorithm, matrix sizes less than $7 \times 7$ should be chosen. The proposed algorithm is impacted by the acquisition time, where the acquisition time of $1 \mathrm{~min}$ showed the biggest error compared to $3 \mathrm{~min}$ and $5 \mathrm{~min}$ scan times (Figures 5.7 and 5.8). 
Furthermore, our algorithm as designed determines not only the $S G$ which contains the total activity compensated for spill-out, but also obtains the FV. By simply dividing the total activity by the total number of voxels belonging to FV (VO) we can obtain the mean activity concentration (mAC). This gives a better measurement of the RC of a tumor without the need to estimate the PSF of the camera; it was able to correct for the $\mathrm{RC}$ just by obtaining the $\mathrm{VO}$. The $\mathrm{RC}$ is usually measured using the maximum activity of a lesion [15, 49], but in this algorithm it is able to achieve the RC based on the mAC. RC is used to evaluate the accuracy of AC. The AC is used for measuring $\mathrm{SUV}_{\text {mean }}$ and $\mathrm{SUV}_{\max }$. The SUVmax is based on the one pixel with maximum intensity making it vulnerable to noise and it is erroneous to use it for heterogeneous tumors. On the other hand, the $\mathrm{SUV}_{\text {mean }}$ is based on average intensity of all the voxels belonging to tumor and its accuracy is dependent on FV. The $\mathrm{SUV}_{\text {mean }}$ measured by our algorithm is unique compared to any of the published algorithms because it uses PVE corrected FV and blur included total activity to measure mAC. The $\mathrm{SUV}_{\text {mean }}$ derived from our algorithm is a better method to calculate SUV in clinical images compared to $\mathrm{SUV}_{\max }$.

Our algorithm has shown that the RC value as well as an FV estimate can be obtained automatically. De Bernardi et al. presented an algorithm similar to our's in the sense that they were able to apply segmentation and PVE correction together, but the algorithm was applied during reconstruction of an image as compared to the proposed algorithm which is applied during post-processing [32], and therefore the algorithm could be applied on an image provided by a clinician that has already been reconstructed. De Bernardi et al. used the camera's measured PSF to account for the spill-out of activity outside of the tumor. The use of a camera's PSF or a user defined PSF sets a great 
limitation on these previously reported algorithms [14] since the PSF may not be known or not be obtainable by the clinician providing the reconstructed image set.

\subsubsection{CONCLUSION}

The algorithm developed has many unique features like the ability to measure mean activity concentration and the usage of deconvolution to segment the tumor in addition to correcting for PVE. The ability of the algorithm to generate consistent results upon varying multiple parameters demonstrates its robustness to withstand the complexity encountered in real clinical images.

\subsection{PHYSICAL AND SIMULATED PHANTOM STUDIES (HETEROGENEOUS TUMORS)}

\subsubsection{INTRODUCTION}

Thus far the algorithm was tested on tumors with homogeneous activity profile, which have much lower complexity than clinical data. The studies described in this chapter explore the case of heterogeneous tumors. The IDTS algorithm has two stopping conditions, the second stopping condition developed considering that there could be heterogeneity within the tumors. For the studies described herein, heterogeneous tumors were generated by both physical and simulated phantoms. In the physical phantom studies the heterogeneous tumors were also irregular shaped making the segmentation even more challenging, whereas in the simulated phantom data spherical phantom tumors were still used. 


\subsubsection{FV OF IRREGULAR SHAPED HETEROGENEOUS PHYSICAL TUMORS}

The physical phantom used for irregular shaped phantom data is called LiquiPhil phantom. The LiquiPhil phantom is provided with irregular shaped inserts in which we can place the smaller spheres in order to mimic irregular shaped heterogeneous tumors as shown in Figure 5.11. The volume of the organs are large $(92-145 \mathrm{ml})$ and of the spheres $(0.5-8 \mathrm{ml})$. The irregular shaped heterogeneous activity simulated tumors were placed inside the phantom to better represent a realistic clinical case. The irregular shaped physical tumors had cores of different sizes $(1,2,4$ and $8 \mathrm{ml})$ which could be filled with activity. The specifications of the heterogeneous tumors are given in Table 5.4 (below).

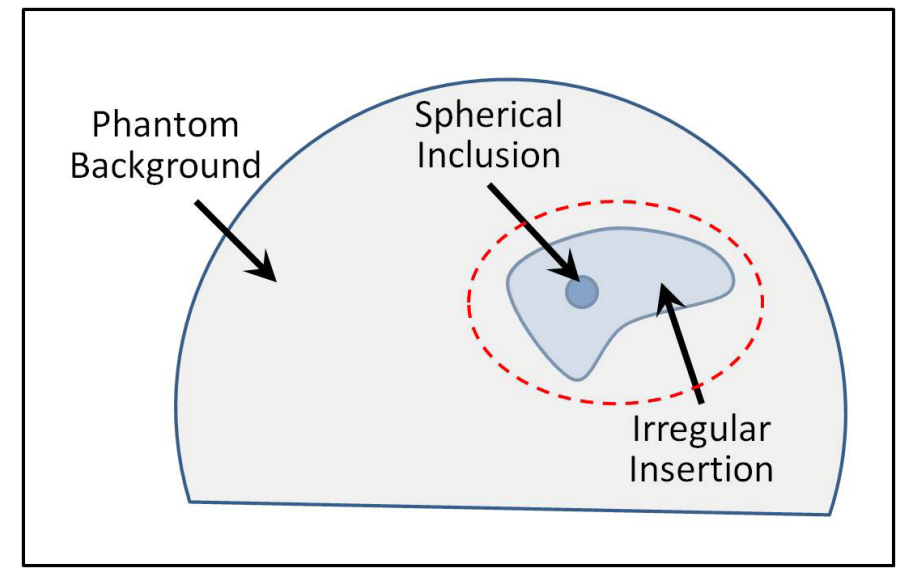

Figure 5.11 Schematic of a phantom with an irregular shaped heterogeneous physic tumor (indicated by the red dotted circle). 
Table 5.4 Specifications of the irregular shaped heterogeneous physical tumor phantom experiments.

\begin{tabular}{lcccc}
\hline \hline Number & $\begin{array}{c}\text { Activity ratio between } \\
\text { tumor and core }\end{array}$ & $\begin{array}{l}\text { Activity ratio } \\
\text { between tumor and } \\
\text { background }\end{array}$ & $\begin{array}{c}\text { Core volume } \\
(\mathrm{ml})\end{array}$ & $\begin{array}{c}\text { Tumor } \\
\text { volume (ml) }\end{array}$ \\
\hline Tumor1 & $3: 1$ & $8.25: 1$ & 8 & 145 \\
Tumor2 & $3: 0$ & $8.25: 1$ & 4 & 92 \\
Tumor3 & $1: 3$ & $2.75: 1$ & 2 & 137 \\
Tumor4 & $1: 2$ & $2.75: 1$ & 2 & 137 \\
Tumor5 & $2: 1$ & $5.5: 1$ & 4 & 94 \\
Tumor6 & $2: 0$ & $5.5: 1$ & 8 & 137 \\
\hline
\end{tabular}

. The activity levels in the irregular shaped tumor and spherical insert were kept different from each other in order to achieve heterogeneity. The algorithm was tested on these irregular shaped physical tumors (Figure 5.12). The tumors are visible in Figure 5.12.a and their corresponding segmentation is shown in Figure 5.12.b. 

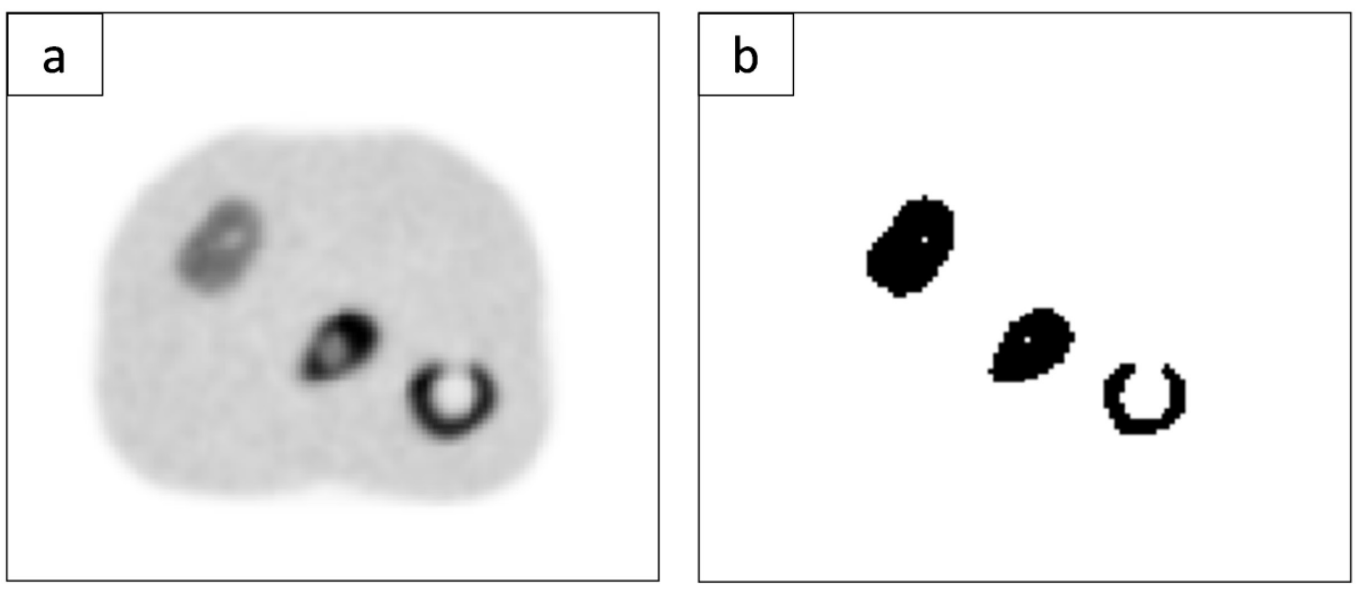

Figure 5.12 Physical tumors with spherical cores of different activity concentrations to represent tumors with heterogeneous uptake distribution. (a) Three tumors with spherical cores, with different activity concentration between the tumors and their cores. The tumor at the right has no activity in the core to simulate necrosis. (b) Segmentation of the three tumors showing that the algorithm is able to segment the necrotic section within the right most tumors.

In Figure 5.13 it is observed that the FV of 5 out of 6 tumors is very close to the true volume, but in tumor 3 there is an underestimation in FV of $\sim 10 \%$. The FWHM was $6 \mathrm{~mm}$ and the matrix size of $5 \times 5$ was used during the application of IDTS algorithm. The error bar shows variation due to different acquisition times ( $1 \mathrm{~min}, 3 \mathrm{~min}$ and $5 \mathrm{~min}$ ). Figure 5.13.a and Figure 5.13.b show FV for phantom data acquired at pixel sizes of 5.46 $\mathrm{mm}$ and $3.46 \mathrm{~mm}$ respectively. For tumors with necrotic cores the HT algorithm was able to segment the core from the tumor in some of the slices, but failed to do so in the slices where the area of the core was small and highly affected by PVE, that is, where the intensity within the core was greater than the background intensity. 


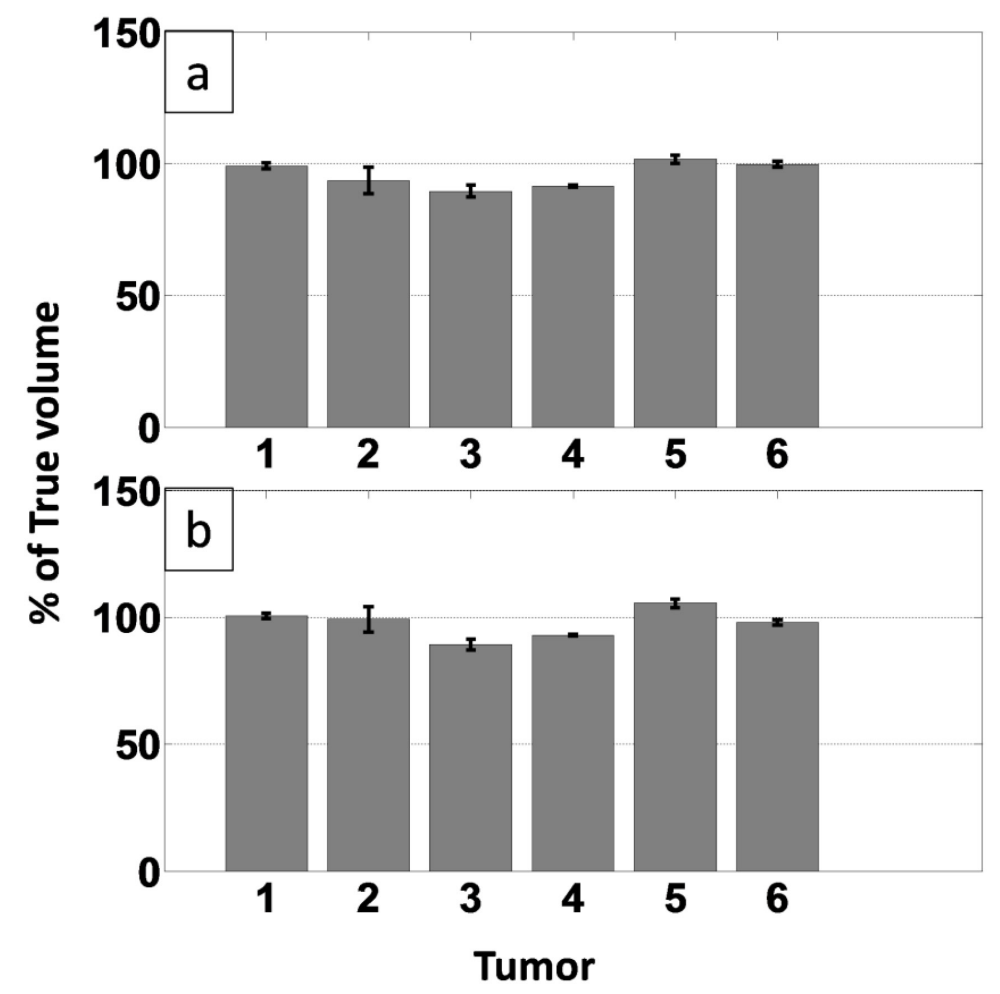

Figure 5.13 The percentage of volume from irregular shaped heterogeneous simulated tumors. (a) pixel size $5.46 \mathrm{~mm}$ and (b) pixel size $3.64 \mathrm{~mm}$. The FWHM is kept at $6 \mathrm{~mm}$ and matrix size is $5 \times 5$. Error bars correspond to the variation due to different image acquisition times or image noise ( $5 \mathrm{~min}, 3 \mathrm{~min}$ and $1 \mathrm{~min}$ ). 


\subsubsection{COMPUTER SIMULATED PHANTOM DATA}

The algorithm was tested on simulated data, where the tumors are incorporated within the NCAT phantom.. Tumors manually segmented from a clinical ${ }^{18}$ F-FDG -PET image and given pixel intensity similar to what is observed in that clinical image was placed within the liver in the NCAT simulated image. The tumor volume was $51 \mathrm{ml}$, with the volume of each voxel being $0.03 \mathrm{ml}$. The size of the image was $128 \times 128 \times 128$. To simulate heterogeneous uptake, a second region of volume $6.87 \mathrm{ml}$ was placed within the tumor. The second region formed the heterogeneous core of the tumor, where different activity concentrations of the tumor and the core could be maintained. Gaussian noise was added with a mean of zero and variance of 0.001 . The image was convolved with a Gaussian PSF (FWHM of $6.5 \mathrm{~mm}$ ) to simulate blur similar to the one observed in images that are derived from the PET camera.

The simulated data, with known ground truth, helps to provide voxel to voxel accuracy of the segmented tumor. The NCAT program was used to simulate nuclear emission images of the abdomen in which a tumor with heterogeneous uptake was placed inside the liver. Activity concentration ratios of $6: 10$ and 8:6 between tumor and its core were chosen, and are referred to as Tumor 1 and Tumor 2, respectively. The image of Tumor 1 is shown in Figure 5.14. 


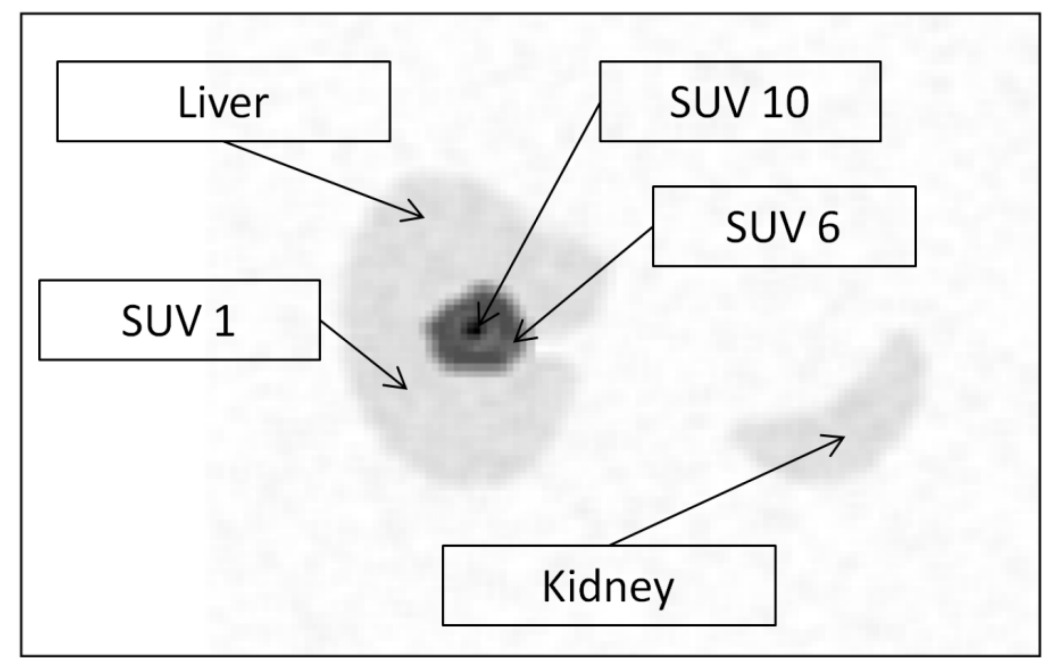

Figure 5.14 NCAT simulated phantom where a heterogeneous tumor is incorporated inside the liver, SUV of large tumor $(51 \mathrm{ml})$ is 6 and the SUV of the heterogeneous section $(6.8 \mathrm{ml})$ of the tumor is 9 . The SUV of the liver and kidney is kept at 1 .

The actual volume of the tumor was $51 \mathrm{ml}$ and the percentage of true volume $(\%$ of true volume), given by Equation 5.1 and classification error (CE) were estimated. CE is a function of two types of errors; one is called positive classification error (PCE), where voxels of the background are made part of the tumor and the other is called negative classification error (NCE), where voxels of the tumor are made part of the background (Equation 5.3).

$$
\mathrm{CE}=\frac{\mathrm{PCE}+\mathrm{NCE}}{V O} \times 100
$$


The percentage of true volume ( $\%$ of true volume) only gives the correlation between the numbers of voxels in the segmented tumor to the true number of voxels. But $\mathrm{CE}$ also provides spatial correlation of each voxel, which can be used as an extra statistic to measure the performance of the algorithm [2]. The results of the CE and percentage of true volume (\% of true volume) estimation with the different FWHM Gaussian PSF in the deconvolution stage of IDTS algorithm is provided in Table 5.5 (below). 
Table 5.5 Percentage of true volume (\% of True Volume) and classification error (CE) of the algorithm in segmenting NCAT simulated heterogeneous tumors. The algorithm is applied by changing FWHM from $4 \mathrm{~mm}, 6 \mathrm{~mm}, 8 \mathrm{~mm}$ to $10 \mathrm{~mm}$. The matrix size of PSF is kept at $5 \times 5$.

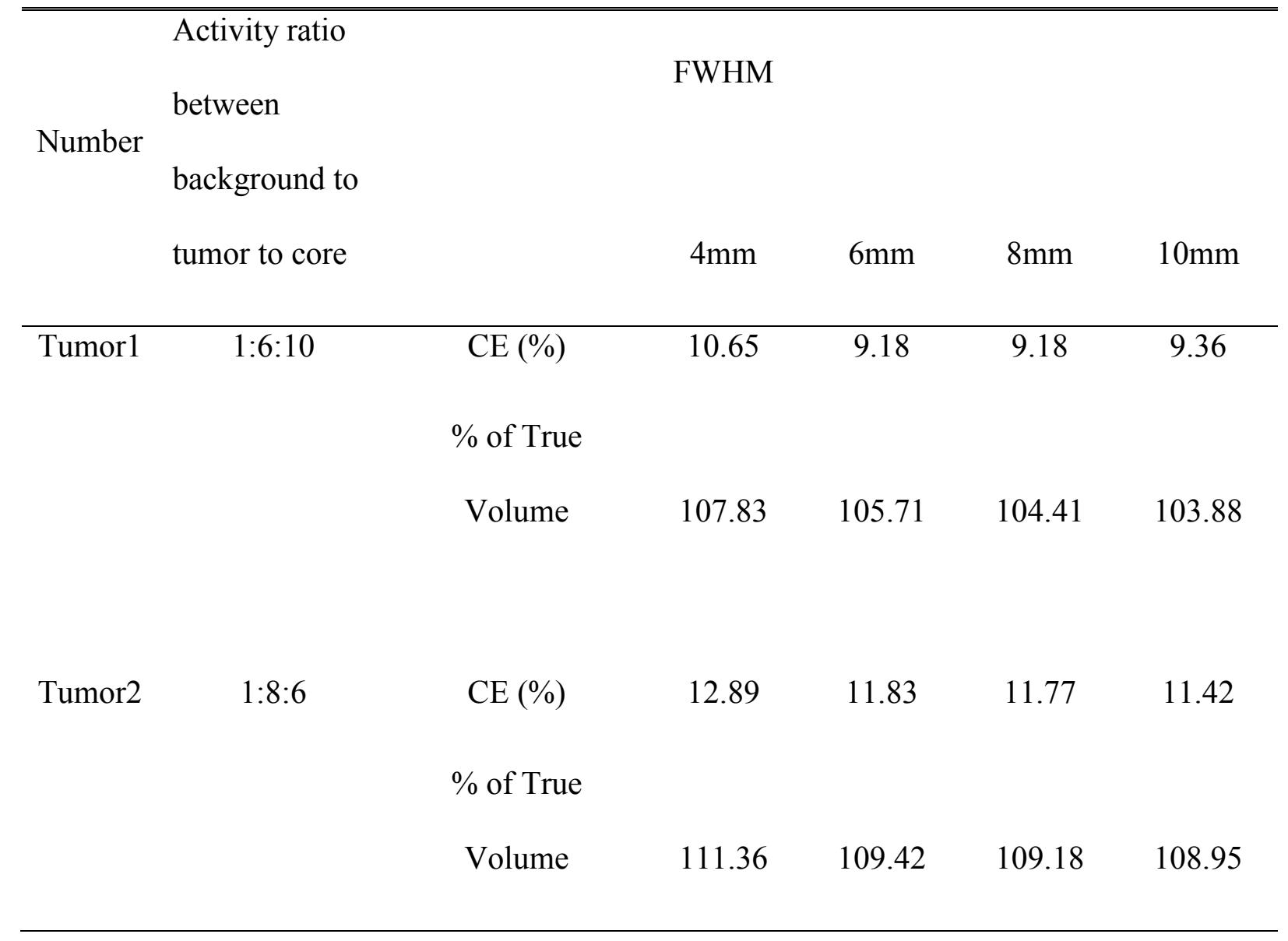

\subsubsection{DISCUSSION}

The computer simulated data used for testing the proposed algorithm is not as realistic as the simulated data used by Hatt et al [44]. Hatt et al. used the Monte Carlo based PET simulating software package GATE, which helps to include the variation of the camera's geometry and reconstruction algorithm [44, 45]. In this dissertation 
Gaussian noise and Gaussian blur were added to NCAT data to simulate PET images with PVE. The simulated data presented by Belhassen et al. was used as a guide to generate simulated data in this study [42]. Belhassen et al. used NCAT data with Poisson noise added but without any addition of blur [42]. Interestingly, with the proposed algorithm, the FV estimations for the physical phantom experiments were better than those for the computer simulated phantom, but the differences were very small. The results of the $\%$ of true volume and CE using the computer simulated phantom are around $10 \%$ for both Tumor 1 and Tumor 2, which is comparable to other algorithms cited in this dissertation. Nevertheless the algorithm should be further tested on more realistic simulated data. The RC of the hetereogeneous uptake tumors were not measured for both physical phantom and simulated phantom. The explanation is that to measure the $\mathrm{RC}$ of hetereogeneous uptake tumors would require measuring FV of different heterogeneous sections within the tumor as well as their corresponding AC. Hatt et al. and Belhassen et al. were able to segment tumors which have inhomogeneous uptake [42, 45]. Hatt et al. were able to segment regions within the tumor. Yang et al. did segmentation of heterogeneous region within the tumor. They used simulated data generated using SimSet, which is still more advanced then my simulated data [60]. The proposed algorithm was tested against tumors which have inhomogeneous uptake, but it did not segment the regions within a tumor. Segmenting a region within the tumor (also called sub-volume segmentation) could be a helpful feature that would be added to the IDTS algorithm. Sub-volume segmentation enables the administration of adaptive modulating radiation dose based on the criteria of whether the sub-volumes are necrotic or hypoxic. 
The algorithm still needs to be tested on an open source database of simulated PET data to test its performance and also compare the results to published algorithms [61]..

\subsubsection{CONCLUSION}

In this chapter, our algorithm was used to segment heterogeneous tumors, which is important because the activity profile of a heterogeneous tumor is closer to that of a real tumor. The algorithm should always be tested on physical and simulated phantom data as both have their advantages and disadvantages. The advantage of the physical phantom is that the noise and blur are similar to real images. The disadvantage is that it lacks ground truth (exact location and activity of a tumor) and while the amount of activity can be varied, the shape and size remains fixed. The advantage of a simulated phantom is that it provides the ground truth and there is no limit to how much the tumor size, shape and activity could be varied; the disadvantage is that the noise and blur are not as realistic as the physical phantom. . 


\subsection{ALGORITHM APPLIED ON CLINICAL DATA}

\subsubsection{INTRODUCTION}

Until now the algorithm has been tested on phantom data only, both physical and simulated. In order for the algorithm to be acceptable it must be demonstrated successfully on clinical images. We have applied our algorithm on 10 clinical data sets all with hepatocellular carcinoma. The size and shape of each tumor is different. There are some tumors which also have necrosis within them. This is a complex set of data with which to test the performance of the iterative deconvolution thresholding segmentation (IDTS) algorithm. There is no definitive way to validate the accuracy of an algorithm in the clinical data, however. The gold standard that is considered here is manual segmentation by a radiologist. To validate our algorithm we are comparing it with manual segmentation and see if the results match or correlate. Also to give a better perspective, the algorithm is compared to a fixed threshold method.

\subsubsection{RESULTS OF THE CLINICAL DATA}

To do the manual segmentation, the radiologist was required to draw manually a region of interest (ROI) around each tumor on each slice. The time required by the radiologist was around 2-3 three hours on average for each data set. Once the ROIs were drawn they were compared with the manual segmentation and the error in the volume was measured. The fixed threshold methods were also compared with the manual segmentation method. The two fixed threshold methods used were 1) T50, which uses $50 \%$ of the maximum value as the threshold, and 2) T60 which uses $60 \%$ of the maximum value as the threshold (Figure 5.15 below). The mean activity concentration 
(mAC) from the manual segmented tumor was also compared with mAC from IDTS algorithm, T50 and T60.

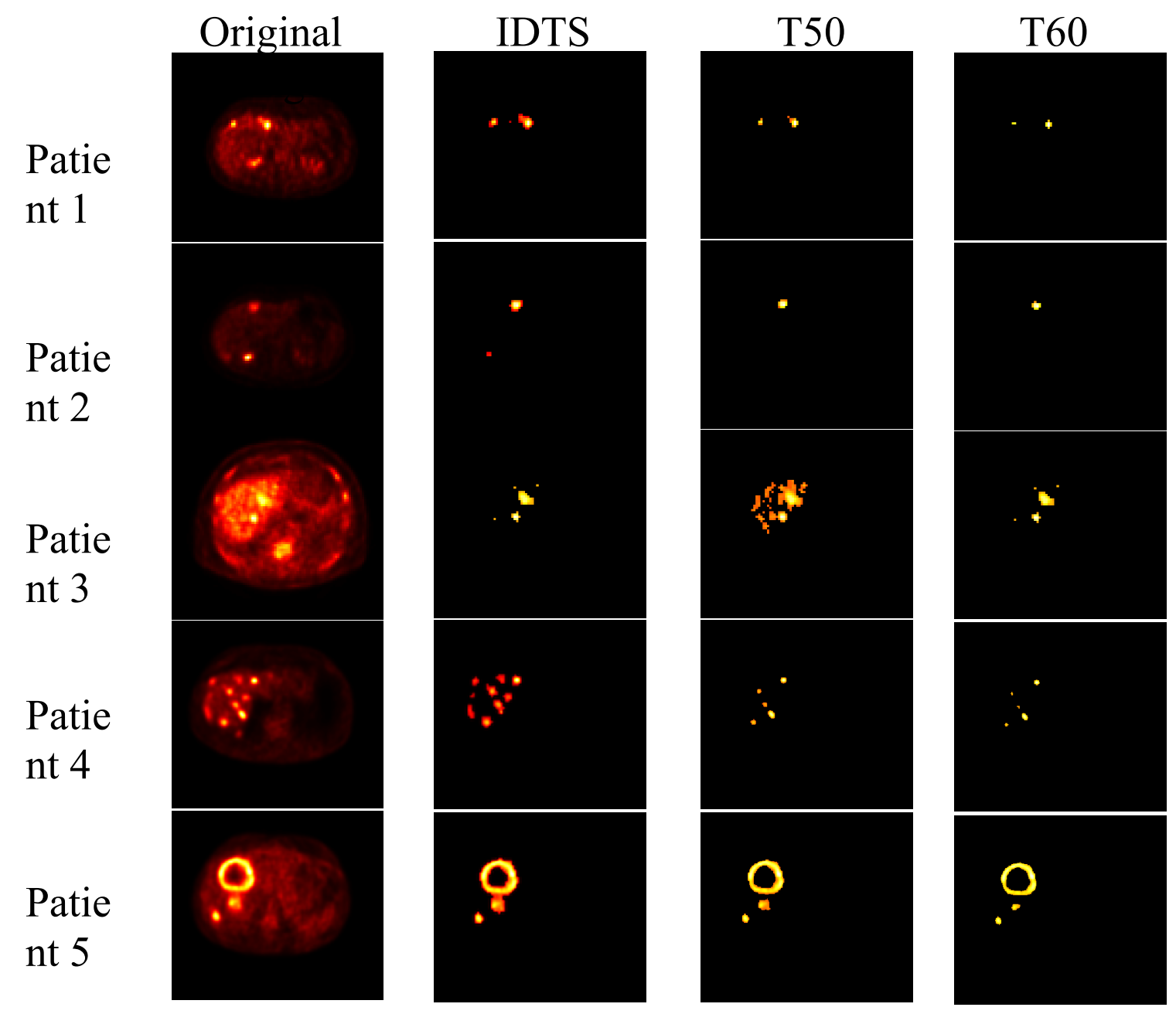

Figure 5.15 The results from three methods of segmentation on 5 out of total 10 patient data sets: column 1 shows the original image and column 2 shows the output of the IDTS algorithm, and columns 2 and 3 give results of the application of T50 and T60 segmentation methods, respectively. 
Visually observing Figure 5.15, we can say that the T50 and T60 methods have failed to accurately segment the tumors in most cases. The quantitative comparison of the IDTS algorithm, T50 and T60 to manual segmentation is given in Table 5.6 (below). The "Percentage of volume error" is the percentage difference between estimated FV (calculated using algorithms) and the manually segmented volume. Similarly "Percentage of $\mathrm{mAC}$ error" is the difference between estimated $\mathrm{mAC}$ and the $\mathrm{mAC}$ derived from manual segmentation.

Table 5.6 Results showing percentage of volume error and percentage of mean activity concentration (mAC) error of IDTS algorithm, T50 and T60 when compared to manual segmentation

\begin{tabular}{ccc}
\hline \hline Method & Percentage of volume error & Percentage of mAC error \\
\hline IDTS algorithm & $-17 \%+/-9 \%$ & $15 \%+/-11 \%$ \\
T50 & $-60 \%+/-33 \%$ & $20 \%+/-13 \%$ \\
T60 & $-73 \%+/-47 \%$ & $23 \%+/-16 \%$ \\
\hline
\end{tabular}




\subsubsection{DISCUSSION}

. The most accurate way to validate the clinical image is to compare it with a histological specimen to ensure that the volume is actually tumor. The histological specimen is taken by surgically removing the tumor and measuring the volume and comparing the volume with pre-surgery PET scans [62]. Such a comparison is not possible in our study as the volumes were measured of tumors which were not resectable, so manual segmentation was the only method available for the data to be compared. In our study the results were compared to manual segmentation done by a radiologist giving a comparison judgment.. There have been studies where the functional tumor volumes (FVs) were compared to manual segmentation in CT images [48, 63, 64]. The CT images are a good choice for comparison but have several drawbacks as well. CT images within a PET machine are not of high resolution whereas using stand-alone CT images would require co-registration of the images. For the best comparison, contrast CT images should be used as it is easy to manually segment the tumor in them. Still CT images cannot show necrosis within the tumor. In Figure 5.15, for patient 5 we see the tumors have necrosis and thus a comparison between PET and CT images will never match. Considering all these facts the manual segmentation was performed on PET images. One thing that the radiologist considered is that the images are blurred and our algorithm finds partial volume effect corrected (PVE) functional volume (FV) which removes blur. So, all the manual segments were drawn with the intention of removing blur and the background around the tumor. The fixed threshold method is still used by radiologists to segment clinical data $[33,65]$. In order to provide a point of reference and objectivity, the results from the IDTS algorithm were shown here with the T50 and T60 method. Fixed 
thresholding cannot compensate for the background activity and different signal to background ratio which have the greatest effect on the results. The T50 and T60 consistently underestimated the $\mathrm{FV}$, and overestimated the $\mathrm{mAC}$ because they were able to segment pixels with very high intensity and remove the low-intensity pixels which were part of the tumor. There have been publications where the range of threshold from $30=70 \%$ of the maximum was used $[37,40,66,67]$, hence the T50 and T60 methods were chosen here.

\subsubsection{CONCLUSION}

The segmentation results of the IDTS algorithm are very promising when compared with manual segmentation results. There were 10 patient data sets used with a total of 40 tumors, giving a large enough data set for comparison. The use of more numbers of data sets would be very valuable to further test the algorithm.

\subsection{PHANTOM DATA WITH DIFFERENT RECONSTRUCTION PARAMETERS}

\subsubsection{INTRODUCTION}

The reconstruction parameters play an important role as changes in them can affect the output of functional volume (FV) and mean activity concentration (mAC). We tested our algorithm with changes in the number of iterations and subset of maximum likelihood ordered subset expectation maximization (ML-OS-EM) iterative reconstruction algorithm. 


\subsubsection{RESULTS OF DIFFERENT RECONSTRUCTION PARAMETER}

The changes of FV are shown in Figures 5.16-5.19 where the different reconstruction parameters are used. Four reconstruction parameters are used: 8 subset $\times 1$ iterations, 8 subset $\times 2$ iterations, 8 subset $\times 8$ iterations and 18 subset $\times 4$ iterations.

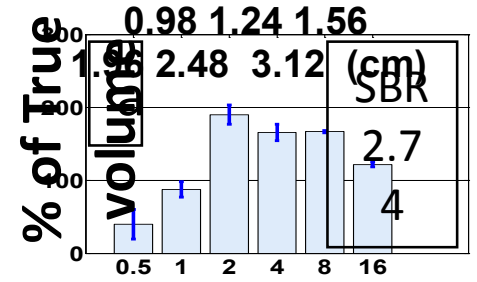

$$
\begin{aligned}
& \text { Recon } \\
& \text { Parameter } 8 \\
& \text { subsets and } 1 \\
& \text { iteration }
\end{aligned}
$$
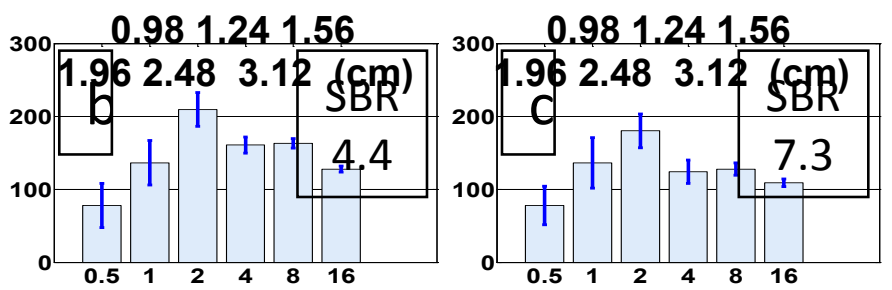

Volume (ml)

Figure 5.16 Results of FV estimation with reconstruction parameter 8 subset $\times 1$ iterations, for different signal to background ratio (SBR) (a) 2.74 (b) 4.4 and (c) 7.3. The FWHM of the point spread function was kept at $6 \mathrm{~mm}$ and matrix size $5 \times 5$ and the acquisition time was $5 \mathrm{~min}$.

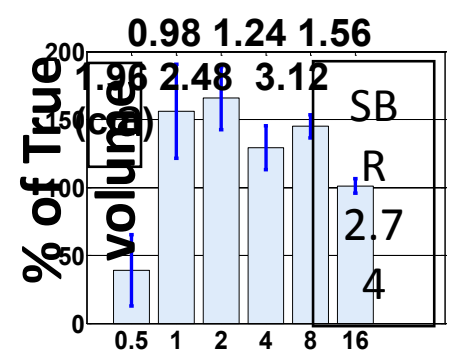

$$
\begin{array}{|l|}
\hline \text { Recon } \\
\text { Parameter } 8 \\
\text { subsets and } 2 \\
\text { iterations }
\end{array}
$$
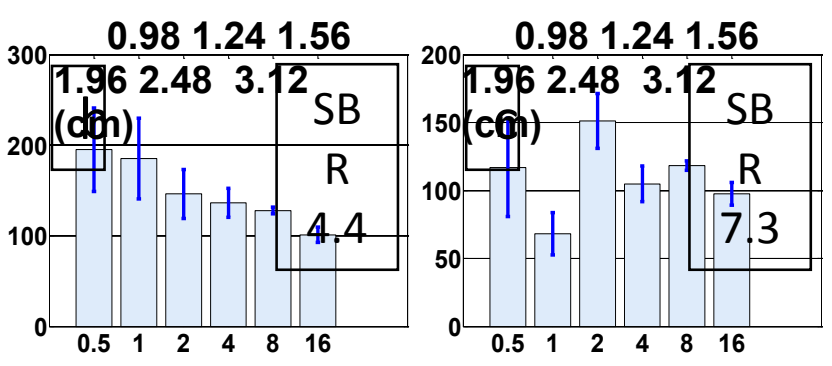

Volume (ml) 
Figure 5.17 Results of FV estimation with reconstruction parameter 8 subset $\times 2$ iterations, for different signal to background ratio (SBR) (a) 2.74 (b) 4.4 and (c) 7.3. The FWHM of the point spread function was kept at $6 \mathrm{~mm}$ and matrix size $5 \times 5$ and the acquisition time was $5 \mathrm{~min}$.
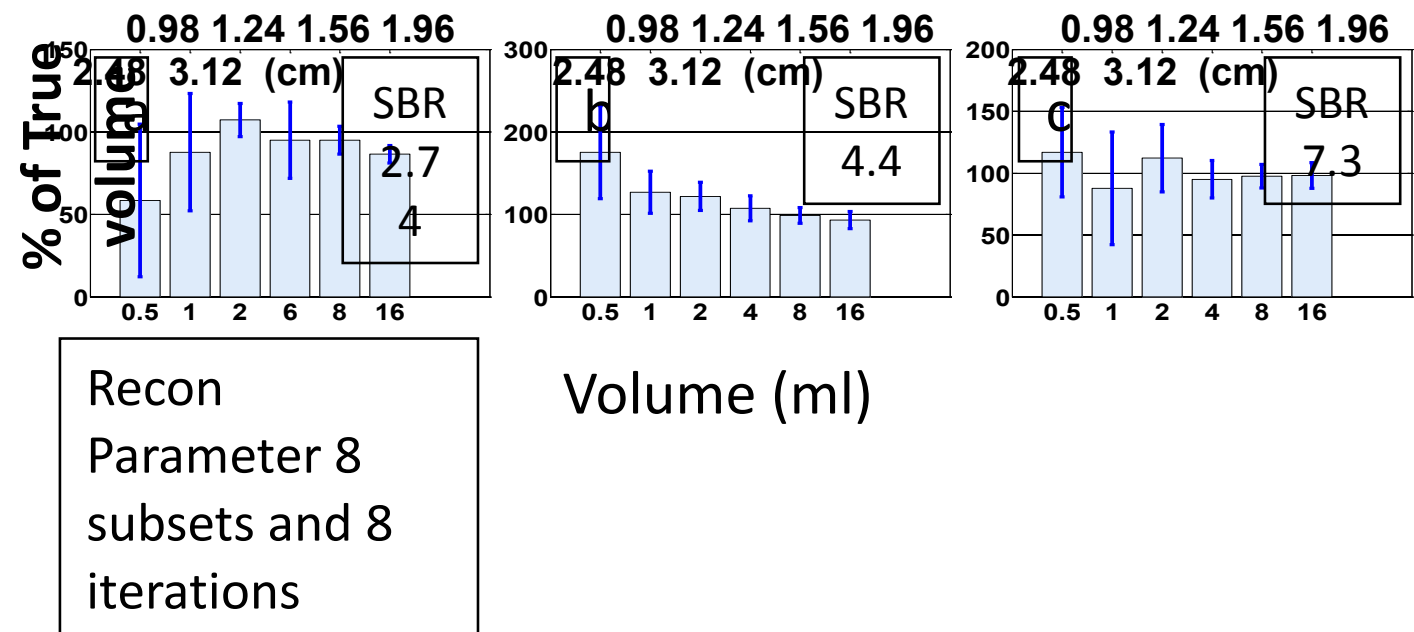
Figure 5.18 Results of $\mathrm{FV}$ estimation with reconstruction parameter 8 subset $\times 8$ iterations, for different signal to background ratio (SBR) (a) 2.74 (b) 4.4 and (c) 7.3. The FWHM of the point spread function was kept at $6 \mathrm{~mm}$ and matrix size $5 \times 5$ and the acquisition time was 5 min.
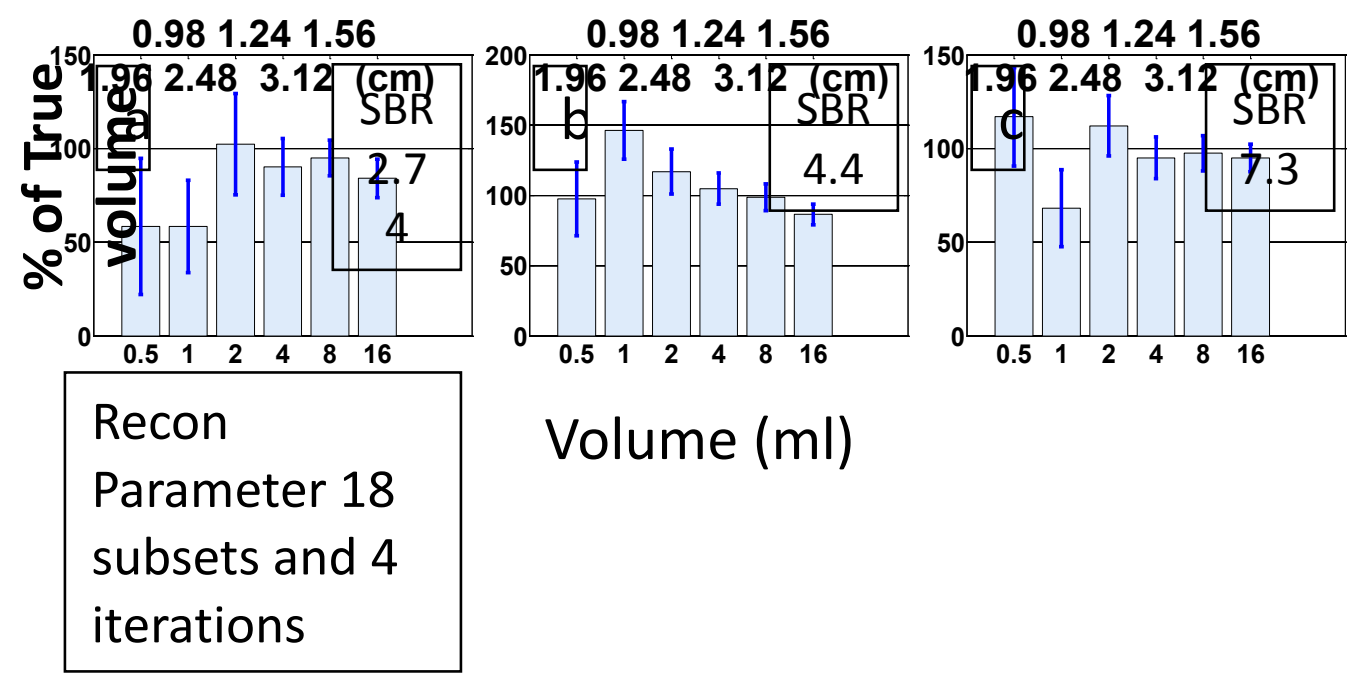

Figure 5.19 Results of FV estimation with reconstruction parameter 18 subset $\times 4$ iterations, for different signal to background ratio (SBR) (a) 2.74 (b) 4.4 and (c) 7.3. The FWHM of the point spread function was kept at $6 \mathrm{~mm}$ and matrix size $5 \times 5$ and the acquisition time was $5 \mathrm{~min}$.

In Figure 5.16 we can see that the performance of the algorithm is very poor for $0.5 \mathrm{ml}$ sphere at SBR 2.74 but the performance of the algorithm improves as the sphere size increases. Also from Figures 5.17-5.19 we see that by increasing the number of iterations the FV estimation also improves.

In Chapter 3 we gave the details of altered histogram thresholding (HT) algorithm. The changes were made to the HT algorithm in the iterative deconvolution thresholding 
segmentation (IDTS) algorithm to accommodate for the possibility of heterogeneous background in clinical data. The altered HT algorithm was re-applied on the physical phantom data in order to determine if the $\mathrm{FV}$ and $\mathrm{mAC}$ vary greatly compared to the results in section 5.1. The parameters in the algorithm were kept constant and the values of the full width half maximum of the point spread function was kept at $6 \mathrm{~mm}$ and matrix size of the PSF was $5 \times 5$, the scan time was kept at $5 \mathrm{~min}$. These parameters were chosen based on the results from section 5.1 as these parameters give the best results. In Figure 5.20 the results of $\mathrm{FV}$ estimation and $\mathrm{mAC}$ are given, where '\% of True Volume' and 'Recovery Coefficient' formula is given in Equations 5.1 and 5.2.
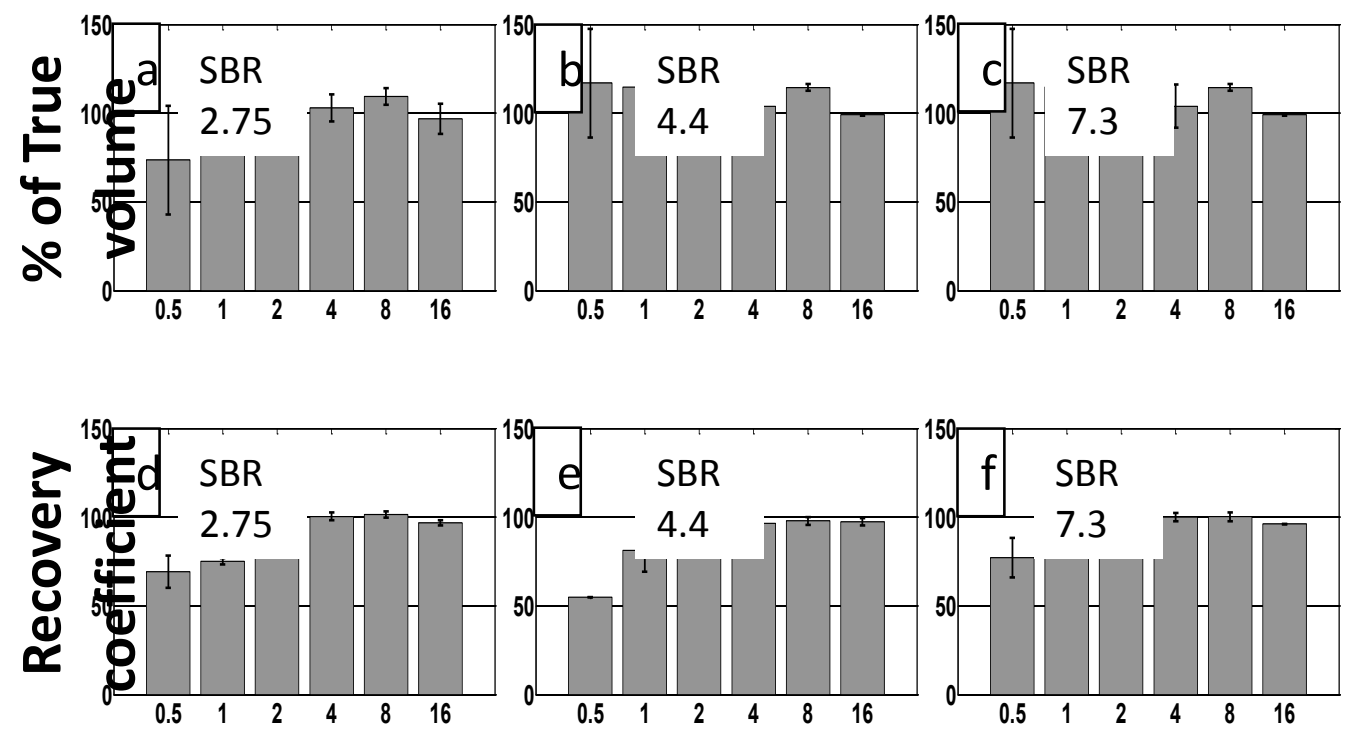

\section{Volume (ml)}


Figure 5.20 FV and $\mathrm{mAC}$ estimation after of altered histogram thresholding (HT) algorithm.

The algorithm was modified for clinical images but its performance was also tested on the phantom data. We do not see much difference in FV estimation and in some cases it is negligible.

\subsubsection{DISCUSSION}

The algorithm is tested by varying the parameters of the reconstruction algorithm. Hatt et al. performed a study by changing the cameras but not changing parameters within the camera to test the impact on the FV estimation [68]. The reconstruction parameters usually are optimized to achieve the best images. The poor results for the reconstruction parameter of 8 subset $\times 1$ iterations does not affect the performance of the algorithm as this parameter are unlikely to be set for a camera. We intended to test the limit of how well the algorithm can perform, so reconstructing with more than one iteration demonstrated that the algorithm performance is unaffected.

\subsubsection{CONCLUSION}

The iterative deconvolution thresholding segmentation (IDTS) algorithm has been thoroughly tested on physical phantom data, simulated data and clinical data. The robustness and reliability of the results will help building more confidence within the radiologist. 


\section{CHAPTER 6}

Summary

\subsection{OVERALL DISCUSSION AND SIGNIFICANCE OF THE WORK}

The algorithm developed is unique compared to any other published algorithm developed to date. Our algorithm accomplished tumor segmentation, volume quantification and partial volume effect (PVE) correction. This unique feature makes the algorithm a complete tumor quantification algorithm. As mentioned in the Chapters 2 many groups have tried to achieve tumor segmentation and PVE correction but as per our knowledge none have achieved both together. The functional volume (FV) and mean activity concentration (mAC) are functions of each other. Hence it is important that we quantify both FV and $\mathrm{mAC}$ in the same algorithm. Both FV and $\mathrm{mAC}$ have been used as prognostic indicators to predict the success of a treatment $[2-6,11-13,25]$. The mAC is used to calculate standard uptake value (SUV); the SUV is based on mAC and is called $\mathrm{SUV}_{\text {mean }}$. Another way to calculate SUV is by using the maximum intensity of a tumor,

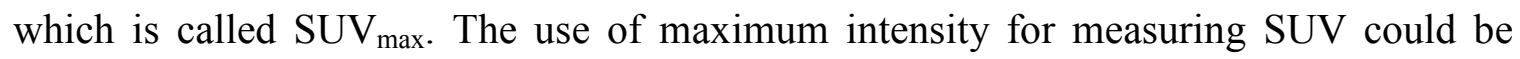
risky as one pixel cannot quantify the entire tumor. If the tumors have heterogeneity then using $\mathrm{SUV}_{\max }$ would produce an incorrect result. PET images also suffer from noise, so basing the SUV on one pixel might result in calculations using that noisy point. To avoid the noise, another method that can be used to calculate the SUV is using peak values $\left(\mathrm{SUV}_{\text {peak }}\right)$ which involves using some percentage of high intensity pixels so that the problem of using one pixel is avoided.The use of mAC to calculate $\mathrm{SUV}_{\text {mean }}$ is only 
possible if the images are PVE corrected.. If the images are not PVE corrected the $\mathrm{SUV}_{\text {mean }}$ would be less when compared to PVE corrected $\mathrm{SUV}_{\text {mean }}$. The $\mathrm{SUV}_{\text {mean }}$ measurement requires knowledge of the $\mathrm{FV}$, where the activity of all the pixels are summed and divided by the FV. As PVE affects both the activity within the tumor and the activity due to blurring, FV is also affected and the error in the non PVE corrected SUV could be doubled.

The SUV measurement of our algorithm is unique in that it accounts for tumor activity and blur activity and divides by FV. There have been recent publications by several groups where they account for both tumor activity and blur activity for SUV measurement; however, they have not shown the accuracy of their FV $[69,70]$. Our algorithm demonstrated superior performance on small tumors when tested on physical phantom data compared to any other published algorithm. The algorithm has potential to become a "one-stop shop" for tumor quantification with capability of segmenting tumors and measuring PVE corrected FV and mAC. The algorithm was tested with different signal to background ratios, different levels of noise and different reconstruction parameters. The demonstrated robustness will allow the algorithm to be applied on data acquired from different cameras. Using one algorithm to perform analysis in multicenter studies will give better correlation among different data sets.

Additionally, the algorithm is computationally inexpensive, making it ideal for the analysis of a large number of data sets. The algorithm has the potential to greatly increase the efficacy of dose calculation, and treatment planning could be adapted for every tumor. Accuracy of our FV estimation could help reduce the toxicity among patients by 
allowing the radiologist to focus the treatment precisely within the tumor margins with minimized damage to surrounding healthy tissues.

\subsection{FUTURE WORK}

The iterative deconvolution theresholding segmentation (IDTS) algorithm developed herein is currently applied on 2D images slice by slice, once in the transverse view and then in the coronal view. The intersection of the two volumes is considered as the final volume. Ideally, the algorithm should be completely $3 \mathrm{D}$ to perform even faster computation. The 2D application of the algorithm causes segmentation of some spurious pixels which require further processing to be removed. The IDTS algorithm uses 256 bins in the histogram thresholding (HT) algorithm and the final result is dependent on choosing the number of bins. To make the algorithm more robust it has to be further improved and if possible made independent of the choice of the number of bins used.

The graphical user interface (GUI) for the application of IDTS algorithm is still in its developmental stage and the implementation of GUI will make the algorithm user friendly and easy to set-up in a clinic. The volume of interest (VOI) generating algorithm has only been applied on the liver tumor segmentation, it has to be tested on tumors from other organs. Radiation therapy is widely used in head, neck, and prostate cancer so segmentation of tumors in those organs should also be tested.

Furthermore, the algorithm should be tested against a simulated PET image database [61]. Herein, the algorithm was tested on clinical data from 10 patients; one of the future goals will be to test the algorithm on a larger patient dataset and determine if the output from the algorithm allows to clinician to make better decisions and ultimately 
improve clinical outcomes. Thus, it would be useful to quantify tumor pre-treatment and post-treatment and see if the functional volume (FV) and mean activity concentration (mAC) correlates with the dose estimated.

Another place where the algorithm can potentially be used is to correct for blurring due to respiration. Because of the fast computation of our algorithm, it can potentially be combined with respiratory gating to provide better tumor quantification. 


\section{REFERENCES}

[1] "Cancer Facts \& Figures 2012," American Cancer Society, Atlanta2012.

[2] K. P. Chu, J. D. Murphy, T. H. La, T. E. Krakow, A. Iagaru, E. E. Graves, A. Hsu, P. G. Maxim, B. Loo, D. T. Chang, and Q. T. Le, "Prognostic Value of Metabolic Tumor Volume and Velocity in Predicting Head-and-Neck Cancer Outcomes," International Journal of Radiation Oncology Biology Physics, vol. 83, pp. 1521-1527, Aug 2012.

[3] S. A. Gulec, R. R. Suthar, T. C. Barot, and K. Pennington, "The prognostic value of functional tumor volume and total lesion glycolysis in patients with colorectal cancer liver metastases undergoing Y-90 selective internal radiation therapy plus chemotherapy," European Journal of Nuclear Medicine and Molecular Imaging, vol. 38, pp. 1289-1295, Jul 2011.

[4] I. Hoseok, K. Kim, S. J. Kim, I. J. Kim, K. Pak, and H. Kim, "Prognostic value of metabolic volume measured by F-18 FDG PET-CT in patients with esophageal cancer," Thoracic Cancer, vol. 3, pp. 255-261, Aug 2012.

[5] K. Manohar, B. R. Mittal, A. Bhattacharya, P. Malhotra, and S. Varma, "Prognostic value of quantitative parameters derived on initial staging F-18fluorodeoxyglucose positron emission tomography/computed tomography in patients with high-grade non-Hodgkin's lymphoma," Nuclear Medicine Communications, vol. 33, pp. 974-981, Sep 2012.

[6] V. S. Nair, O. Gevaert, G. Davidzon, S. Napel, E. E. Graves, C. D. Hoang, J. B. Shrager, A. Quon, D. L. Rubin, and S. K. Plevritis, "Prognostic PET F-18-FDG Uptake Imaging Features Are Associated with Major Oncogenomic Alterations in Patients with Resected Non-Small Cell Lung Cancer," Cancer Research, vol. 72, pp. 3725-3734, Aug 2012.

[7] P. B. Romesser, M. M. Qureshi, B. A. Shah, L. T. Chatburn, S. Jalisi, A. K. Devaiah, R. M. Subramaniam, and M. T. Truong, "Superior prognostic utility of gross and metabolic tumor volume compared to standardized uptake value using $\mathrm{PET} / \mathrm{CT}$ in head and neck squamous cell carcinoma patients treated with intensity-modulated radiotherapy," Annals of Nuclear Medicine, vol. 26, pp. 527534, Aug 2012.

[8] C. Tang, J. D. Murphy, B. Khong, T. H. La, C. Kong, N. J. Fischbein, A. D. Colevas, A. H. Iagaru, E. E. Graves, B. W. Loo, and Q. T. Le, "Validation that Metabolic Tumor Volume Predicts Outcome in Head-and-Neck Cancer," International Journal of Radiation Oncology Biology Physics, vol. 83, pp. 15141520, Aug 2012. 
[9] J. Yoo, J. Y. Choi, S. H. Moon, D. S. Bae, S. B. Park, Y. S. Choe, K. H. Lee, and B. T. Kim, "Prognostic Significance of Volume-Based Metabolic Parameters in Uterine Cervical Cancer Determined Using F-18-Fluorodeoxyglucose Positron Emission Tomography," International Journal of Gynecological Cancer, vol. 22, pp. 1226-1233, Sep 2012.

[10] H. H. W. Chen, N. T. Chiu, W. C. Su, H. R. Guo, and B. F. Lee, "Prognostic Value of Whole-Body Total Lesion Glycolysis at Pretreatment FDG PET/CT in Non-Small Cell Lung Cancer," Radiology, vol. 264, pp. 559-566, Aug 2012.

[11] H. H. Chung, H. W. Kwon, K. W. Kang, N. H. Park, Y. S. Song, J. K. Chung, S. B. Kang, and J. W. Kim, "Prognostic Value of Preoperative Metabolic Tumor Volume and Total Lesion Glycolysis in Patients with Epithelial Ovarian Cancer," Annals of Surgical Oncology, vol. 19, pp. 1966-1972, Jun 2012.

[12] S. R. Liao, B. C. Penney, K. Wroblewski, H. Zhang, C. A. Simon, R. Kampalath, M. C. Shih, N. Shimada, S. Chen, R. Salgia, D. E. Appelbaum, K. Suzuki, C. T. Chen, and Y. Pu, "Prognostic value of metabolic tumor burden on F-18-FDG PET in nonsurgical patients with non-small cell lung cancer," European Journal of Nuclear Medicine and Molecular Imaging, vol. 39, pp. 27-38, Jan 2012.

[13] S. R. Liao, B. C. Penney, H. Zhang, K. Suzuki, and Y. L. Pu, "Prognostic Value of the Quantitative Metabolic Volumetric Measurement on 18F-FDG PET/CT in Stage IV Nonsurgical Small-cell Lung Cancer," Academic Radiology, vol. 19, pp. 69-77, Jan 2012.

[14] D. L. Barbee, R. T. Flynn, J. E. Holden, R. J. Nickles, and R. Jeraj, "A method for partial volume correction of PET-imaged tumor heterogeneity using expectation maximization with a spatially varying point spread function," Physics in Medicine and Biology, vol. 55, pp. 221-236, Jan 2010.

[15] M. Soret, S. L. Bacharach, and I. Buvat, "Partial-volume effect in PET tumor imaging," Journal of Nuclear Medicine, vol. 48, pp. 932-945, Jun 2007.

[16] C. Allison, "Yttrium-90 microspheres (TheraSphere and SIR-Spheres) for the treatment of unresectable hepatocellular carcinoma," Issues Emerg Health Technol, vol. 102, pp. 1-6, 2007.

[17] R. J. Lewandowski and R. Salem, "Yttrium-90 radioembolization of hepatocellular carcinoma and metastatic disease to the liver," Semin Intervent Radiol, vol. 23, pp. 64-72, 2006.

[18] K. Memon, R. J. Lewandowski, A. Riaz, and R. Salem, "Yttrium 90 microspheres for the treatment of hepatocellular carcinoma," Recent Results Cancer Res, vol. 190, pp. 207-24, 2013. 
[19] J. Metyko, J. M. Williford, W. Erwin, J. Poston, Jr., and S. Jimenez, "Long-lived Impurities of 90Y-labeled Microspheres, TheraSphere and SIR-Spheres, and the Impact on Patient Dose and Waste Management," Health Phys, vol. 103, 2012.

[20] M. D'Arienzo, P. Chiaramida, L. Chiacchiararelli, A. Coniglio, R. Cianni, R. Salvatori, A. Ruzza, F. Scopinaro, and O. Bagni, "Y-90 PET-based dosimetry after selective internal radiotherapy treatments," Nuclear Medicine Communications, vol. 33, pp. 633-640, Jun 2012.

[21] A. Kennedy, D. Coldwell, B. Sangro, H. Wasan, and R. Salem, "Radioembolization for the Treatment of Liver Tumors General Principles," American Journal of Clinical Oncology-Cancer Clinical Trials, vol. 35, pp. 9199, Feb 2012.

[22] O. N. Kucuk, C. Soydal, S. Lacin, E. Ozkan, and S. Bilgic, "Selective intraarterial radionuclide therapy with Yttrium-90 (Y-90) microspheres for unresectable primary and metastatic liver tumors," World Journal of Surgical Oncology, vol. 9, Aug 2011.

[23] L. Prompers, J. Bucerius, B. Brans, Y. Temur, L. Berger, and F. M. Mottaghy, "Selective internal radiation therapy (SIRT) in primary or secondary liver cancer," Methods, vol. 55, pp. 253-257, Nov 2011.

[24] S. A. Gulec, G. Mesoloras, W. A. Dezarn, P. McNeillie, and A. S. Kennedy, "Safety and efficacy of Y-90 microsphere treatment in patients with primary and metastatic liver cancer: The tumor selectivity of the treatment as a function of tumor to liver flow ratio," Journal of Translational Medicine, vol. 5, Mar 2007.

[25] S. M. Piduru, D. M. Schuster, B. J. Barron, R. Dhanasekaran, D. H. Lawson, and H. S. Kim, "Prognostic Value of F-18-Fluorodeoxyglucose Positron Emission Tomography-Computed Tomography in Predicting Survival in Patients with Unresectable Metastatic Melanoma to the Liver Undergoing Yttrium-90 Radioembolization," Journal of Vascular and Interventional Radiology, vol. 23, pp. 943-948, Jul 2012.

[26] M. Zalom, R. Yu, M. Friedman, C. Bresee, and A. Waxman, "FDG PET/CT as a Prognostic Test After Y-90 Radioembolization in Patients With Metastatic Hepatic Disease," Clinical Nuclear Medicine, vol. 37, pp. 862-865, Sep 2012.

[27] I. Zerizer, A. Al-Nahhas, D. Towey, P. Tait, B. Ariff, H. Wasan, G. Hatice, N. Habib, and T. Barwick, "The role of early F-18-FDG PET/CT in prediction of progression-free survival after Y-90 radioembolization: comparison with RECIST and tumour density criteria," European Journal of Nuclear Medicine and Molecular Imaging, vol. 39, pp. 1391-1399, Sep 2012. 
[28] H. H. Hartgrink, E. P. M. Jansen, N. C. T. van Grieken, and C. J. H. van de Velde, "Gastric cancer," The Lancet, vol. 374, pp. 477-490.

[29] S. Devic, N. Tomic, S. Faria, G. Dean, R. Lisbona, W. Parker, C. Kaufman, and E. B. Podgorsak, "Impact of 18FDG-PET/CT on biological target volume (BTV) definition for treatment planning for non-small cell lung cancer patients," Nuclear Instruments \& Methods in Physics Research Section a-Accelerators Spectrometers Detectors and Associated Equipment, vol. 571, pp. 89-92, Feb 1 2007.

[30] E. Malinen, A. Sovik, D. Hristov, O. S. Bruland, and D. R. Olsen, "Adapting radiotherapy to hypoxic tumours," Physics in Medicine and Biology, vol. 51, pp. 4903-4921, Oct 72006.

[31] H. Li, W. L. Thorstad, K. J. Biehl, R. Laforest, Y. Su, K. I. Shoghi, E. D. Donnelly, D. A. Low, and W. Lu, "A novel PET tumor delineation method based on adaptive region-growing and dual-front active contours," Medical Physics, vol. 35, pp. 3711-3721, Aug 2008.

[32] E. De Bernardi, E. Faggiano, F. Zito, P. Gerundini, and G. Baselli, "Lesion quantification in oncological positron emission tomography: A maximum likelihood partial volume correction strategy," Medical Physics, vol. 36, pp. 30403049, Jul 2009.

[33] S. A. Nehmeh, H. El-Zeftawy, C. Greco, J. Schwartz, Y. E. Erdi, A. Kirov, C. R. Schmidtlein, A. B. Gyau, S. M. Larson, and J. L. Humm, "An iterative technique to segment PET lesions using a Monte Carlo based mathematical model," Medical Physics, vol. 36, pp. 4803-4809, Oct 2009.

[34] W. Jentzen, L. Freudenberg, E. G. Eising, M. Heinze, W. Brandau, and A. Bockisch, "Segmentation of PET volumes by iterative image thresholding," Journal of Nuclear Medicine, vol. 48, pp. 108-114, Jan 2007.

[35] M. Moussallem, P. J. Valette, A. Traverse-Glehen, C. Houzard, C. Jegou, and F. Giammarile, "New strategy for automatic tumor segmentation by adaptive thresholding on PET/CT images," J Appl Clin Med Phys, vol. 13, p. 3875, 2012.

[36] M. Pacilio, C. Basile, S. Shcherbinin, F. Caselli, G. Ventroni, D. Aragno, L. Mango, and E. Santini, "An innovative iterative thresholding algorithm for tumour segmentation and volumetric quantification on SPECT images: Monte Carlo-based methodology and validation," Medical Physics, vol. 38, pp. 30503061, Jun 2011. 
[37] E. Prieto, P. Lecumberri, M. Pagola, M. Gomez, I. Bilbao, M. Ecay, I. Penuelas, and J. M. Marti-Climent, "Twelve automated thresholding methods for segmentation of PET images: a phantom study," Physics in Medicine and Biology, vol. 57, pp. 3963-3980, Jun 2012.

[38] M. Wanet, J. A. Lee, B. Weynand, M. De Bast, A. Poncelet, V. Lacroix, E. Coche, V. Gregoire, and X. Geets, "Gradient-based delineation of the primary GTV on FDG-PET in non-small cell lung cancer: A comparison with thresholdbased approaches, CT and surgical specimens," Radiotherapy and Oncology, vol. 98, pp. 117-125, Jan 2011.

[39] L. Drever, W. Roa, A. McEwan, and D. Robinson, "Iterative threshold segmentation for PET target volume delineation," Medical Physics, vol. 34, pp. 1253-1265, Apr 2007.

[40] K. Khamwan, A. Krisanachinda, and C. Pluempitiwiriyawej, "Automated tumour boundary delineation on F-18-FDG PET images using active contour coupled with shifted-optimal thresholding method," Physics in Medicine and Biology, vol. 57, pp. 5995-6005, Oct 2012.

[41] I. El Naqa, D. Yang, A. Apte, D. Khullar, S. Mutic, J. Zheng, J. D. Bradley, P. Grigsby, and J. O. Deasy, "Concurrent multimodality image segmentation by active contours for radiotherapy treatment planning," Medical Physics, vol. 34, pp. 4738-4749, Dec 2007.

[42] S. Belhassen and H. Zaidi, "A novel fuzzy C-means algorithm for unsupervised heterogeneous tumor quantification in PET," Medical Physics, vol. 37, pp. 13091324, Mar 2010.

[43] F. Yang and P. W. Grigsby, "A segmentation framework towards automatic generation of boost subvolumes for FDG-PET tumors: A digital phantom study," Eur J Radiol, Jul 262012.

[44] M. Hatt, C. C. le Rest, A. Turzo, C. Roux, and D. Visvikis, "A Fuzzy Locally Adaptive Bayesian Segmentation Approach for Volume Determination in PET," Ieee Transactions on Medical Imaging, vol. 28, pp. 881-893, Jun 2009.

[45] M. Hatt, C. C. le Rest, P. Descourt, A. Dekker, D. De Ruysscher, M. Oellers, P. Lambin, O. Pradier, and D. Visvikis, "ACCURATE AUTOMATIC DELINEATION OF HETEROGENEOUS FUNCTIONAL VOLUMES IN POSITRON EMISSION TOMOGRAPHY FOR ONCOLOGY APPLICATIONS," International Journal of Radiation Oncology Biology Physics, vol. 77, pp. 301-308, May 2010. 
[46] K. Erlandsson, I. Buvat, P. H. Pretorius, B. A. Thomas, and B. F. Hutton, "A review of partial volume correction techniques for emission tomography and their applications in neurology, cardiology and oncology," Phys Med Biol, vol. 57, pp. 0031-9155, 2012.

[47] N. J. Hoetjes, F. H. van Velden, O. S. Hoekstra, C. J. Hoekstra, N. C. Krak, A. A. Lammertsma, and R. Boellaard, "Partial volume correction strategies for quantitative FDG PET in oncology," Eur J Nucl Med Mol Imaging, vol. 37, pp. 1679-87, 2010.

[48] A. Le Maitre, M. Hatt, O. Pradier, C. Cheze-le Rest, and D. Visvikis, "Impact of the accuracy of automatic tumour functional volume delineation on radiotherapy treatment planning," Phys Med Biol, vol. 57, pp. 5381-97, Sep 72012.

[49] A. S. Kirov, J. Z. Piao, and C. R. Schmidtlein, "Partial volume effect correction in PET using regularized iterative deconvolution with variance control based on local topology," Physics in Medicine and Biology, vol. 53, pp. 2577-2591, May 2008 .

[50] B. K. Teo, Y. Seo, S. L. Bacharach, J. A. Carrasquillo, S. K. Libutti, H. Shukla, B. H. Hasegawa, R. A. Hawkins, and B. L. Franc, "Partial-volume correction in PET: Validation of an iterative postreconstruction method with phantom and patient data," Journal of Nuclear Medicine, vol. 48, pp. 802-810, May 2007.

[51] N. Boussion, C. Cheze Le Rest, M. Hatt, and D. Visvikis, "Incorporation of wavelet-based denoising in iterative deconvolution for partial volume correction in whole-body PET imaging," Eur J Nucl Med Mol Imaging, vol. 36, pp. 1064-75, 2009.

[52] A. S. Dewalle-Vignion, N. Betrouni, R. Lopes, D. Huglo, S. Stute, and M. Vermandel, "A New Method for Volume Segmentation of PET Images, Based on Possibility Theory," Ieee Transactions on Medical Imaging, vol. 30, pp. 409-423, Feb 2011.

[53] W. P. Segars, D. S. Lalush, and B. M. W. Tsui, "Modeling respiratory mechanics in the MCAT and spline-based MCAT phantoms," Ieee Transactions on Nuclear Science, vol. 48, pp. 89-97, Feb 2001.

[54] I. G. Zubal, C. R. Harrell, E. O. Smith, Z. Rattner, G. Gindi, and P. B. Hoffer, "COMPUTERIZED 3-DIMENSIONAL SEGMENTED HUMAN ANATOMY," Medical Physics, vol. 21, pp. 299-302, Feb 1994. 
[55] S. Jan, G. Santin, D. Strul, S. Staelens, K. Assie, D. Autret, S. Avner, R. Barbier, M. Bardies, P. M. Bloomfield, D. Brasse, V. Breton, P. Bruyndonckx, I. Buvat, A. F. Chatziioannou, Y. Choi, Y. H. Chung, C. Comtat, D. Donnarieix, L. Ferrer, S. J. Glick, C. J. Groiselle, D. Guez, P. F. Honore, S. Kerhoas-Cavata, A. S. Kirov, V. Kohli, M. Koole, M. Krieguer, D. J. van der Laan, F. Lamare, G. Largeron, C. Lartizien, D. Lazaro, M. C. Maas, L. Maigne, F. Mayet, F. Melot, C. Merheb, E. Pennacchio, J. Perez, U. Pietrzyk, F. R. Rannou, M. Rey, D. R. Schaart, C. R. Schmidtlein, L. Simon, T. Y. Song, J. M. Vieira, D. Visvikis, R. V. de Walle, E. Wieers, and C. Morel, "GATE: a simulation toolkit for PET and SPECT," Physics in Medicine and Biology, vol. 49, pp. 4543-4561, Oct 2004.

[56] I. Buvat, I. Castiglioni, J. Feuardent, and M. C. Gilardi, "Unified description and validation of Monte Carlo simulators in PET," Physics in Medicine and Biology, vol. 50, pp. 329-346, Jan 2005.

[57] A. Reilhac, G. Batan, C. Michel, C. Grova, J. Tohka, D. L. Collins, N. Costes, and A. C. Evans, "PET-SORTEO: Validation and development of database of simulated PET volumes," Ieee Transactions on Nuclear Science, vol. 52, pp. 1321-1328, Oct 2005.

[58] K. Thielemans, C. Tsoumpas, S. Mustafovic, T. Beisel, P. Aguiar, N. Dikaios, and M. W. Jacobson, "STIR: software for tomographic image reconstruction release 2," Physics in Medicine and Biology, vol. 57, pp. 867-883, Feb 2012.

[59] G. Komar, M. Teras, M. Seppanen, J. Hirvonen, T. Vahlberg, J. Bergman, and H. Minn, "Comparison of 2D and 3D performance for FDG PET with different acquisition times in oncological patients," Nuclear Medicine Communications, vol. 30, pp. 16-24, Jan 2009.

[60] F. Yang and P. W. Grigsby, "A segmentation framework towards automatic generation of boost subvolumes for FDG-PET tumors: A digital phantom study," Eur J Radiol, vol. 26, p. 26, 2012.

[61] S. Tomei, A. Reilhac, D. Visvikis, N. Boussion, C. Odet, F. Giammarile, and C. Lartizien, "OncoPET_DB: A Freely Distributed Database of Realistic Simulated Whole Body 18F-FDG PET Images for Oncology," Ieee Transactions on Nuclear Science, vol. 57, pp. 246-255, Feb 2010.

[62] M. Hatt, C. Cheze-le Rest, A. van Baardwijk, P. Lambin, O. Pradier, and D. Visvikis, "Impact of Tumor Size and Tracer Uptake Heterogeneity in F-18-FDG PET and CT Non-Small Cell Lung Cancer Tumor Delineation," Journal of Nuclear Medicine, vol. 52, pp. 1690-1697, Nov 2011. 
[63] M. Hatt, A. Le Pogam, D. Visvikis, O. Pradier, and C. C. Le Rest, "Impact of Partial-Volume Effect Correction on the Predictive and Prognostic Value of Baseline F-18-FDG PET Images in Esophageal Cancer," Journal of Nuclear Medicine, vol. 53, pp. 12-20, Jan 2012.

[64] M. Hatt, C. C. Le Rest, N. Albarghach, O. Pradier, and D. Visvikis, "PET functional volume delineation: a robustness and repeatability study," European Journal of Nuclear Medicine and Molecular Imaging, vol. 38, pp. 663-672, Apr 2011.

[65] U. Nestle, W. Weber, M. Hentschel, and A. L. Grosu, "Biological imaging in radiation therapy: role of positron emission tomography," Physics in Medicine and Biology, vol. 54, pp. R1-R25, Jan 2009.

[66] X. Bontemps, R. Abgral, C. Leleu, A. Turzo, G. Valette, S. Danhier, P. Y. Le Roux, S. Querellou, G. Potard, R. Marianowski, and P. Y. Salaun, "Comparison of different segmentation methods using PET/CT for HNSCC radiotherapy targeting," Medecine Nucleaire-Imagerie Fonctionnelle Et Metabolique, vol. 36, pp. 303-312, Jun 2012.

[67] O. Suzuki, K. Nishiyama, M. Morimoto, H. Hashiguchi, K. Tanaka, A. Nakajima, S. Nakamura, and K. Yoshino, "Defining PET Standardized Uptake Value Threshold for Tumor Delineation with Metastatic Lymph Nodes in Head and Neck Cancer," Japanese Journal of Clinical Oncology, vol. 42, pp. 491-497, Jun 2012.

[68] M. Hatt, P. Bailly, A. Turzo, C. Roux, and D. Visvikis, "PET functional volume segmentation: a robustness study," in Nuclear Science Symposium Conference Record, 2008. NSS '08. IEEE, 2008, pp. 4335-4339.

[69] F. Hofheinz, J. Langner, J. Petr, B. Beuthien-Baumann, L. Oehme, J. Steinbach, J. Kotzerke, and J. van den Hoff, "A method for model-free partial volume correction in oncological PET," EJNMMI Res, vol. 2, p. 16, Apr 242012.

[70] J. Mertens, A. Dobbeleir, H. Ham, Y. D'Asseler, I. Goethals, and C. Van de Wiele, "Standardized added metabolic activity (SAM): a partial volume independent marker of total lesion glycolysis in liver metastases," European Journal of Nuclear Medicine and Molecular Imaging, vol. 39, pp. 1441-1448, Sep 2012. 


\section{VITA}

\section{RUCHIR BHATT}

Born, Mumbai, India

2001-2006

Bachelors in Engineering

Mumbai University

Mumbai, India

2006-2007

Research Engineer

DS Enterprise

2007-2009

Teaching Assistant

Florida International University

Miami, Florida

2009-2011

Graduate Research Assistant

Florida International University

Miami, Florida

2012

Doctoral Candidate, Dissertation Year Fellow

Florida International University

Miami, Florida

\section{PUBLICATIONS AND PRESENTATIONS}

Ruchir Bhatt, Malek Adjouadi, Mohammed Goryawala, Seza Gulec, Anthony McGoron, "An algorithm for PET tumor volume and activity quantification: Without specifying the camera's point spread function (PSF)," Medical Physics, vol. 39, pp. 4187-4202, Jul 2012. (Original article)

Mohammed Goryawala, Magno R. Guillen, Mercedes Cabrerizo, Seza Gulec, Tushar Barot, Rekha Suthar, Ruchir Bhatt, Anthony Mcgoron, Malek Adjouadi, "A 3D Liver Segmentation Method with Parallel Computing for Selective Internal Radiation Therapy," accepted IEEE - Transactions on Information Technology in Biomedicine. in Press. (Original article)

Mohammed Goryawala, Magno R. Guillen, Armando Barreto, Ruchir Bhatt, Seza Gulec, Tushar Barot, Rekha Suthar, Anthony McGoron, Malek Adjouadi, Design and Evaluation of Parallel Processing Techniques for 3D Liver Segmentation and Volume Rendering, accepted in i-manager's Journal on Software Engineering, Vol 5, No 4, April - June 2011. (Original article) 
Mohammed Goryawala, Magno R. Guillen, Seza Gulec, Tushar Barot, Rekha Suthar, Ruchir Bhatt, Anthony Mcgoron and Malek Adjouadi, "An Accurate 3D Liver Segmentation Method for Selective Internal Radiation Therapy Using a Modified KMeans Algorithm and Parallel Computing", accepted in Int. J. of Innovative Computing Information and Control tentatively to be published in Vol 8, No. 7, July 2012. (Original article)

Rucher Bhatt, Micheal Syme, Mohammed Goryawala, Seza Gulec and Anthony McGoron, "An algorithm for combined segmentation, functional volume (FV) estimation and mean activity concentration (mAC) correction of tumors: A positron emission tomography (PET) study," J NUCL MED MEETING ABSTRACTS 53, 2289- (2012).

Mohammed Goryawala, Seza Gulec, Rekha Suthar, Ruchir Bhatt, Anthony McGoron and Malek Adjouadi, "A low-interaction automatic 3D liver segmentation method for selective internal radiation therapy," J NUCL MED MEETING ABSTRACTS 53, 2251(2012).

Ruchir Bhatt, Mohammed Goryawala, Tushar Barot, Rekha Suthar, Malek Adjouadi, Anthony McGoron, Seza Gulec, "New algorithm for functional volume estimation after partial volume correction: A phantom study”, J Nucl Med. 2010; 51 (Supplement 2):1290

Tushar Barot, Rekha Suthar, Ruchir Bhatt, Mohammed Goryawala, Anthony McGoron, Seza Gulec, "Segmental liver dosimetry integrated comprehensive treatment planning for Y-90 microsphere selective internal radiation therapy in liver cancer", J Nucl Med. 2010; 51 (Supplement 2):12. 